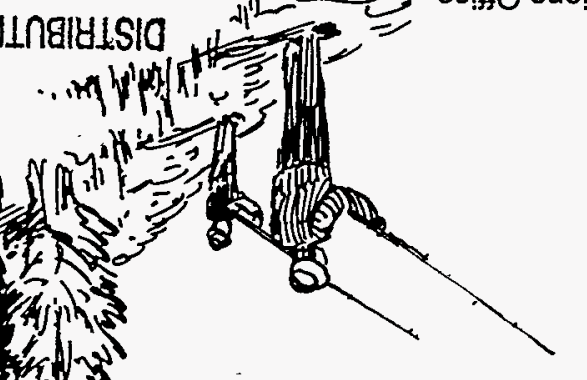

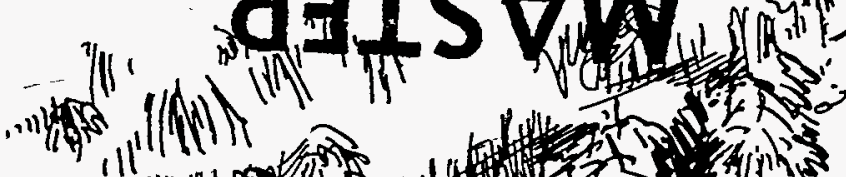

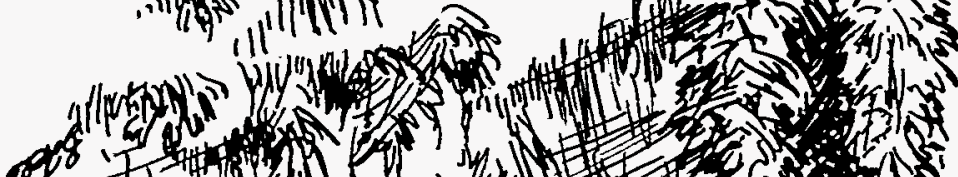
a) (1) N(f)

(1) of 1) (1) $=$ an 积

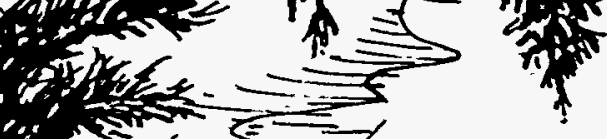

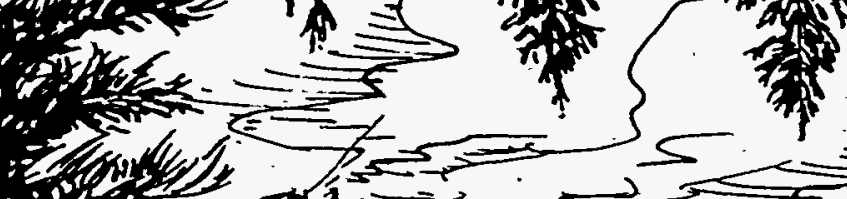
Fition (6) ind

S

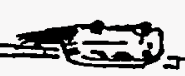

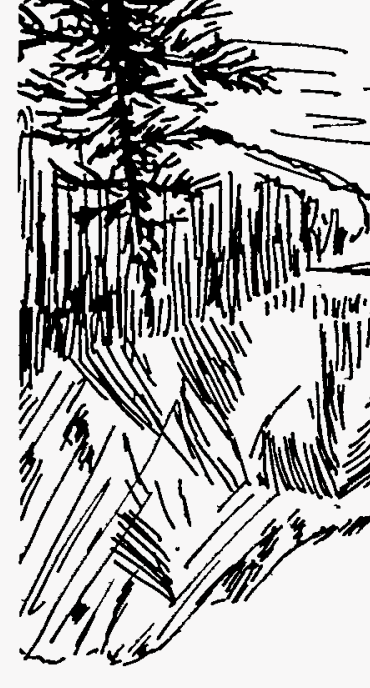

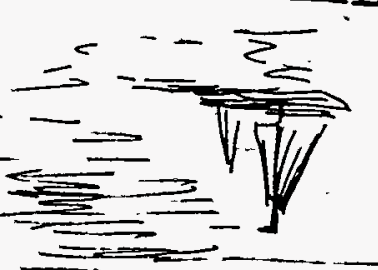
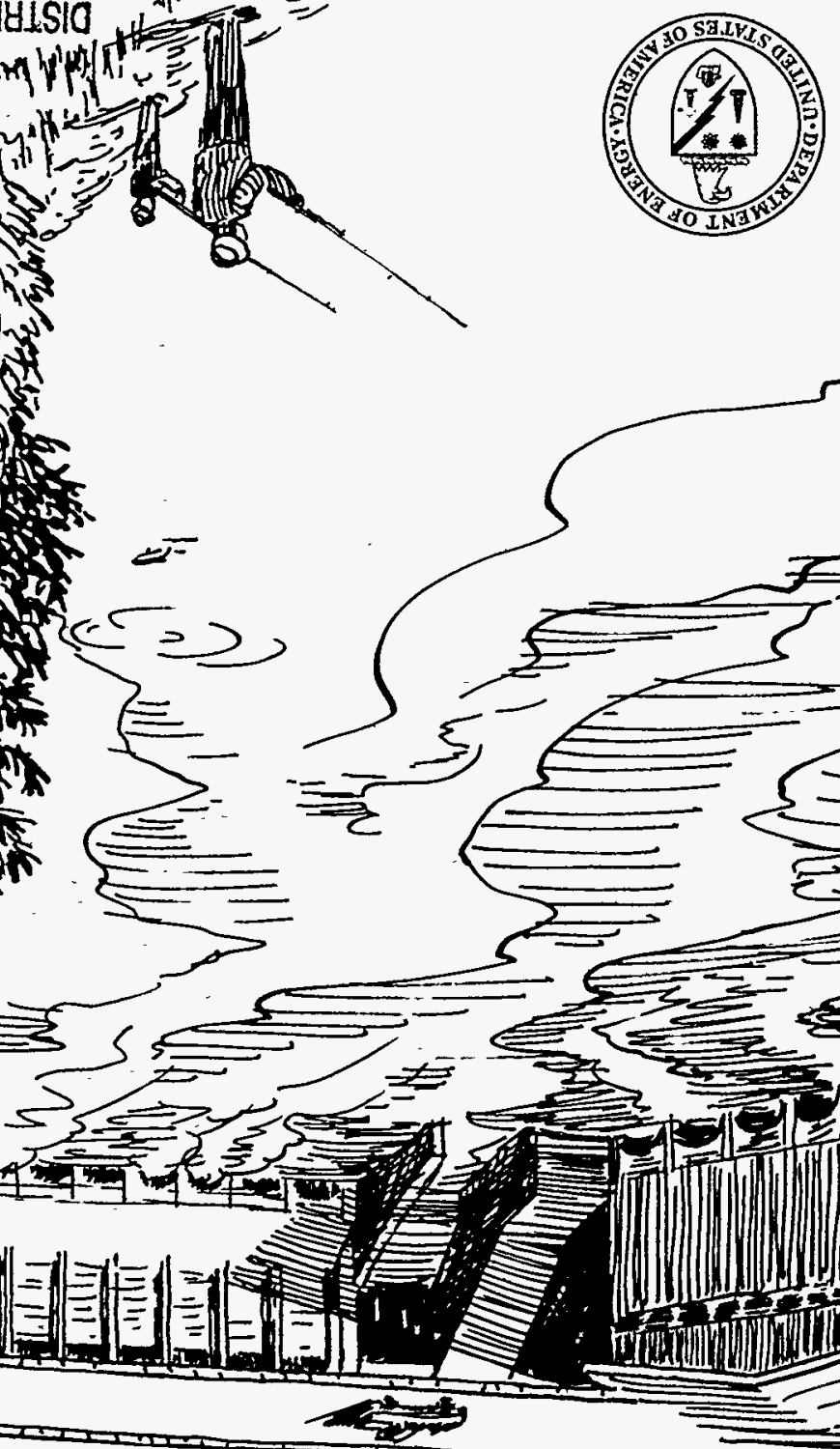


\title{
U.S. Hydropower Resource Assessment for lowa
}

\author{
Prepared by \\ James E. Francfort \\ Project Manager \\ Ben N. Rinehart
}

Published December 1995

\begin{abstract}
Idaho National Engineering Laboratory Renewable Energy Products Department Lockheed Idaho Technologies Company Idaho Falls, Idaho 83415
\end{abstract}




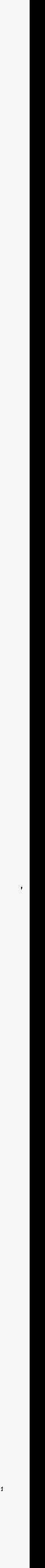




\section{ACKNOWLEDGMENTS}

The author thanks Peggy A.M. Brookshier, John V. Flynn and Jim Alexander of the Department of Energy, and Ward Lenz of the State of Iowa for their active participation and timely comments.

\section{DISCLAIMER}

This report was prepared as an account of work sponsored by an agency of the United States Government. Neither the United States Government nor any agency thereof, nor any of their employees, makes any warranty, express or implied, or assumes any legal liability or responsibility for the accuracy, completeness, or usefulness of any information, apparatus, product, or process disclosed, or represents that its use would not infringe privately owned rights. Reference herein to any specific commercial product, process, or service by trade name, trademark, manufacturer, or otherwise does not necessarily constitute or imply its endorsement, recommendation, or favoring by the United States Government or any agency thereof. The views and opinions of authors expressed herein do not necessarily state or reflect those of the United States Government or any agency thereof. 


\begin{abstract}
The Department of Energy is developing an estimate of the undeveloped hydropower potential in this country. The Hydropower Evaluation Software is a computer model that was developed by the Idaho National Engineering Laboratory for this purpose. The software measures the undeveloped hydropower resources available in the United States, using uniform criteria for measurement. The software was developed and tested using hydropower information and data provided by the Southwestern Power Administration. It is a menu-driven software program that allows the personal computer user to assign environmental attributes to potential hydropower sites, calculate development suitability factors for each site based on the environmental attributes present, and generate reports based on these suitability factors. This report details the resource assessment results for the State of Iowa.
\end{abstract}




\section{CONTENTS}

ACKNOWLEDGMENTS $\ldots \ldots \ldots \ldots \ldots \ldots \ldots \ldots \ldots \ldots \ldots \ldots \ldots \ldots \ldots \ldots \ldots \ldots \ldots \ldots \ldots \ldots \ldots$

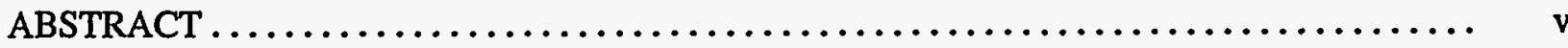

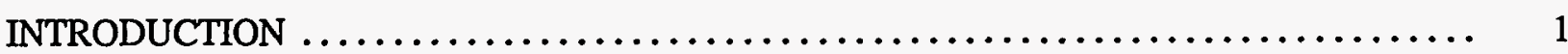

Model Development $\ldots \ldots \ldots \ldots \ldots \ldots \ldots \ldots \ldots \ldots \ldots \ldots \ldots \ldots \ldots \ldots \ldots \ldots \ldots \ldots \ldots \ldots$

Model Goal $\ldots \ldots \ldots \ldots \ldots \ldots \ldots \ldots \ldots \ldots \ldots \ldots \ldots \ldots \ldots \ldots \ldots \ldots \ldots \ldots \ldots$

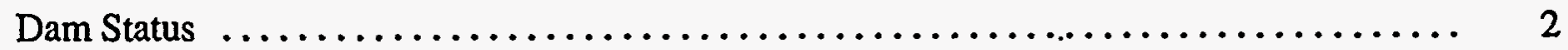

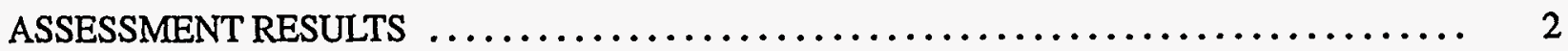

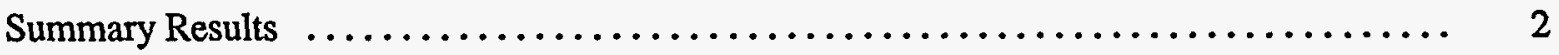

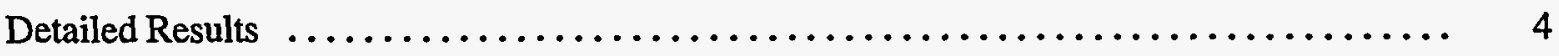

OBTAINING INDIVIDUAL STATE INFORMATION $\ldots \ldots \ldots \ldots \ldots \ldots \ldots \ldots \ldots \ldots$

ADDITIONAL HYDROPOWER EVALUATION SOFTWARE INFORMATION $\ldots \ldots \ldots \ldots \ldots$

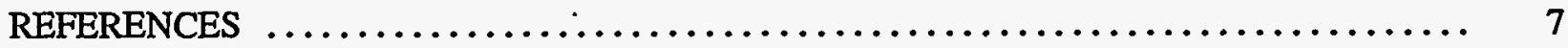

Appendix A-Summary Report $\ldots \ldots \ldots \ldots \ldots \ldots \ldots \ldots \ldots \ldots \ldots \ldots \ldots \ldots \ldots \ldots \ldots \ldots \ldots \ldots \ldots \ldots$

Appendix B-River Basins Report $\ldots \ldots \ldots \ldots \ldots \ldots \ldots \ldots \ldots \ldots \ldots \ldots \ldots \ldots \ldots \ldots \ldots \ldots \ldots$

Appendix C-Iowa Sites Listing $\ldots \ldots \ldots \ldots \ldots \ldots \ldots \ldots \ldots \ldots \ldots \ldots \ldots \ldots \ldots \ldots \ldots \ldots \ldots$

Appendix D-Individual Resource Database Listing $\ldots \ldots \ldots \ldots \ldots \ldots \ldots \ldots \ldots \ldots \ldots$

FIGURES

1. Number of sites with HES-modeled undeveloped hydropower potential $\ldots \ldots \ldots \ldots \ldots \ldots$

2. The HES-modeled undeveloped hydropower potential and the nonmodeled undeveloped hydropower potential $\ldots \ldots \ldots \ldots \ldots \ldots \ldots \ldots \ldots \ldots \ldots$

3. The HES-modeled undeveloped hydropower potential and the total megawatts of HES-modeled undeveloped hydropower potential $\ldots \ldots \ldots \ldots \ldots \ldots \ldots .4$

4. Example of a developed hydroelectric plant in Iowa $\ldots \ldots \ldots \ldots \ldots \ldots \ldots \ldots \ldots$

5. Number of sites with undeveloped hydropower potential in each of the Iowa river basins .... 5

6. Megawatts of HES-modeled undeveloped hydropower potential in the Iowa river basins .... 6

\section{TABLES}

1. Undeveloped hydropower potential summaries for Iowa 



\section{U.S. Hydropower Resource Assessment for \\ lowa}

\section{INTRODUCTION}

In June 1989, the U.S. Department of Energy initiated the development of a National Energy Strategy to identify the energy resources available to support the expanding demand for energy in the United States. Public hearings conducted as part of the strategy development process indicated that undeveloped hydropower resources were not well defined. As a result, the Department of Energy established an interagency Hydropower Resource Assessment Team to ascertain the undeveloped hydropower potential. In connection with these efforts by the Department of Energy, the Idaho National Engineering Laboratory designed the Hydropower Evaluation Software (HES), which is being used to perform a resource assessment of the undeveloped conventional hydropower potential in the United States. This report presents the results of the hydropower resource assessment for the State of Iowa. Undeveloped pumped storage hydropower potential is not included.

The HES was developed as a tool to measure undeveloped hydropower potential regionally or by state. The software is not intended to provide precise development factors for individual sites, but to provide regional or state totals. Because the software was developed as a generic measurement tool encompassing national issues, regional and state totals must be considered judiciously; various local issues may skew undeveloped hydropower potential totals. The information for the resource assessment was compiled from the Federal Energy Regulatory Commission's Hydroelectric Power Resources Assessment database and several other sources. Refer to DOE/ID-10338, the User's Manual (Francfort, Matthews, and Rinehart 1991) for the specifics of the software and to DOE/ID-10430, the Status Report (Francfort, Moore, and Rinehart 1993) for an overview of all resource assessment activities to date.

\section{Model Development}

Hydropower Evaluation Software, both a probability-factor computer model and a database, is a menu-driven software program that is intended to be user-friendly. Computer screens and report generation capabilities were developed to meet the needs of users nationwide. The software uses environmental attribute data to generate an overall project environmental suitability factor (PESF) between 0.1 and 0.9 , where 0.9 indicates the highest likelihood of development and 0.1 indicates the lowest likelihood of development. The suitability factors are dependant on the unique environmental attributes of each potential site. They reflect the considerations that (a) environmental concerns can make a potential site unacceptable, prohibiting its development (for a suitability factor of 0.1 ), or (b) if there are no environmental concerns, there is no effect on the likelihood of site development (for a suitability factor of 0.9). A combination of attributes can result in a lower suitability factor because multiple environmental considerations would reduce the likelihood that a site may be developed to its physical potential.

\section{Model Goal}

The goal of the HES is to assemble an accurate resource database of all sites with undeveloped hydropower potential in the United States for use as a planning tool to determine the viable national hydropower potential. Undeveloped hydropower potential is not limited to the development of new sites; it also includes the development of additional hydropower-generating capacity at sites that currently have hydropower but are not developed to their full potential. This undeveloped hydropower potential is a source of nonpolluting, renewable energy available to meet the growing power needs of the United States. The HES 
should help make this goal obtainable and ensure a set of uniform criteria for national assessment.

\section{Dam Status}

The effects of environmental attributes vary by dam status. The dam status classifications used are

$\mathrm{W}=$ Developed hydropower site with current power generation, but the total hydropower potential has not been fully developed. Only the undeveloped hydropower potential is discussed in this report.

$\mathrm{W} / \mathrm{O}=$ Developed site without current power generation. The site has some type of developed impoundment or diversion structure, but no developed hydropower generating capability.

$\mathrm{U}=$ Undeveloped site. The site does not have power generation capability nor a developed impoundment or diversion structure.

\section{ASSESSMENT RESULTS}

\section{Summary Results}

A total of 79 sites (Table 1) have been identified and assessed for their undeveloped hydropower potential. The HES results for individual site capacities range from 49 kilowatts to 52 megawatts. Most of the sites have potential capacities of under 5 megawatts (Figure 1).

The nonmodeled undeveloped hydropower potential total for Iowa was identified as 455 megawatts. The HES results lowers this estimate about $33 \%$ to 305 megawatts. The greatest reduction in undeveloped hydropower potential occurs at sites with some type of power production already present. These developed sites have an HES-modeled additional undeveloped hydropower potential of 60.7 megawatts, a $47 \%$ reduction in the estimated undeveloped hydropower potential (Figure 2). The number of sites does not change, only the identified undeveloped hydropower potential is reassessed (Figure 3).

The 79 identified sites are located within 7 major river basins and several minor river basins. An example of one developed hydroelectric plant, the Red Rock Lake hydroelectric plant, is shown in Figure 4. The number of sites per major river basin range from 1 each in the Platte River Basin and the Missouri main stream, to 22 sites in the Iowa River Basin (Figure 5). The Iowa River Basin has the most undeveloped hydropower potential ( $99 \mathrm{MW}$ ) of the Iowa river basins (Figure 6).

The 79 identified sites are located within 7 major river basins and several minor river basins. The number of sites per major river basin range from 1 each in the Platte River Basin and the Missouri main stream, to 22 sites in the Iowa River Basin (Figure 5). The Iowa River Basin has the most undeveloped hydropower potential (99 MW) of the Iowariver basins (Figure 6)

Table 1. Undeveloped hydropower potential summaries for Iowa. The table contains the nonmodeled undeveloped name plate potential, as well as the HES-modeled undeveloped hydropower potential totals.

\begin{tabular}{lccc}
\hline & $\begin{array}{c}\text { Number of } \\
\text { projects }\end{array}$ & $\begin{array}{c}\text { Name plate potential } \\
(\mathrm{MW})\end{array}$ & $\begin{array}{c}\text { HES-modeled } \\
\text { potential (MW) }\end{array}$ \\
\cline { 2 - 4 } With power & 7 & 115.5 & 60.7 \\
W/O power & 69 & 310.0 & 219.1 \\
Undeveloped & 3 & 30.0 & 25.0 \\
State Total & 79 & 455.5 & 304.9 \\
\hline
\end{tabular}




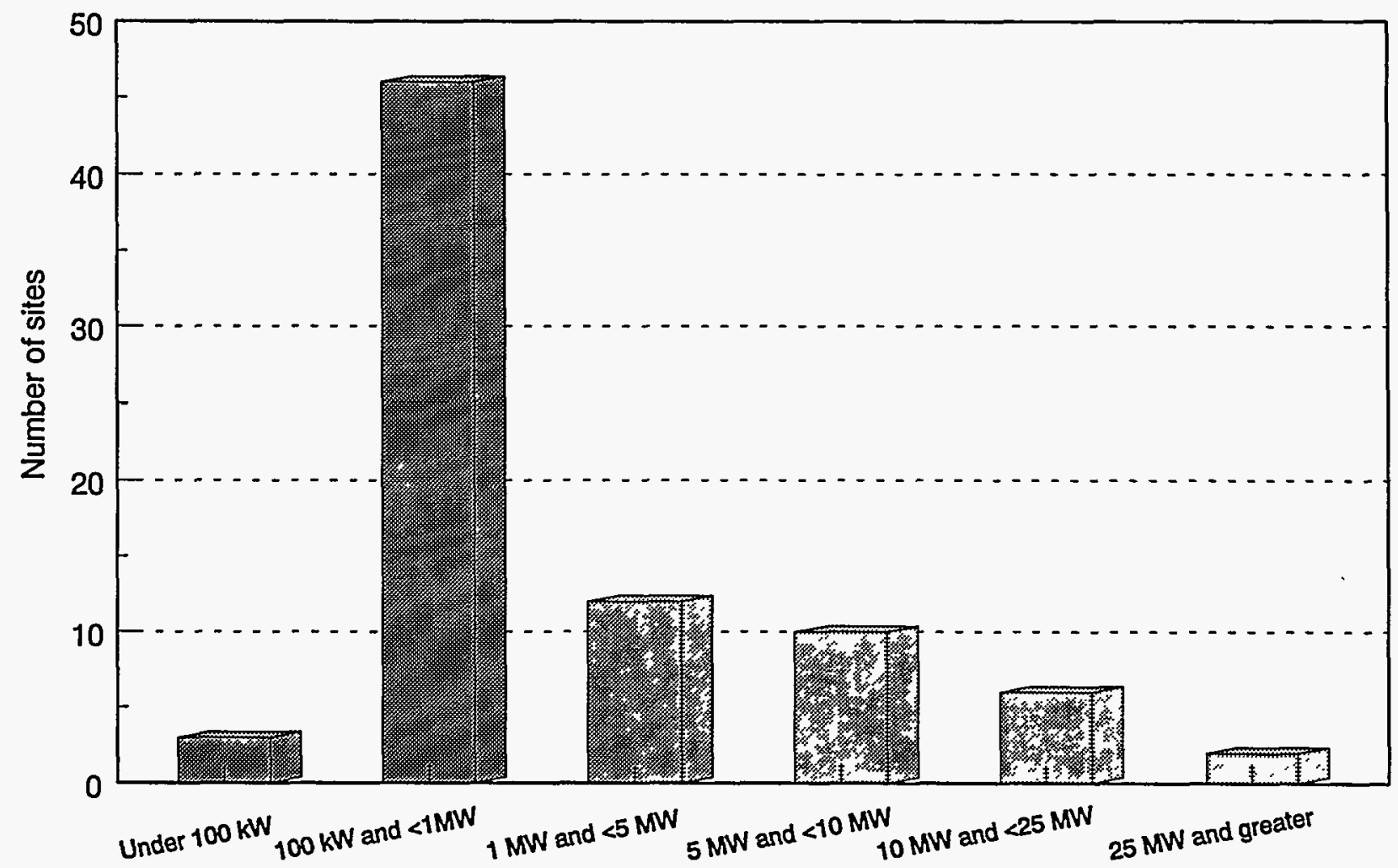

Figure 1. Number of sites with HES-modeled undeveloped hydropower potential.

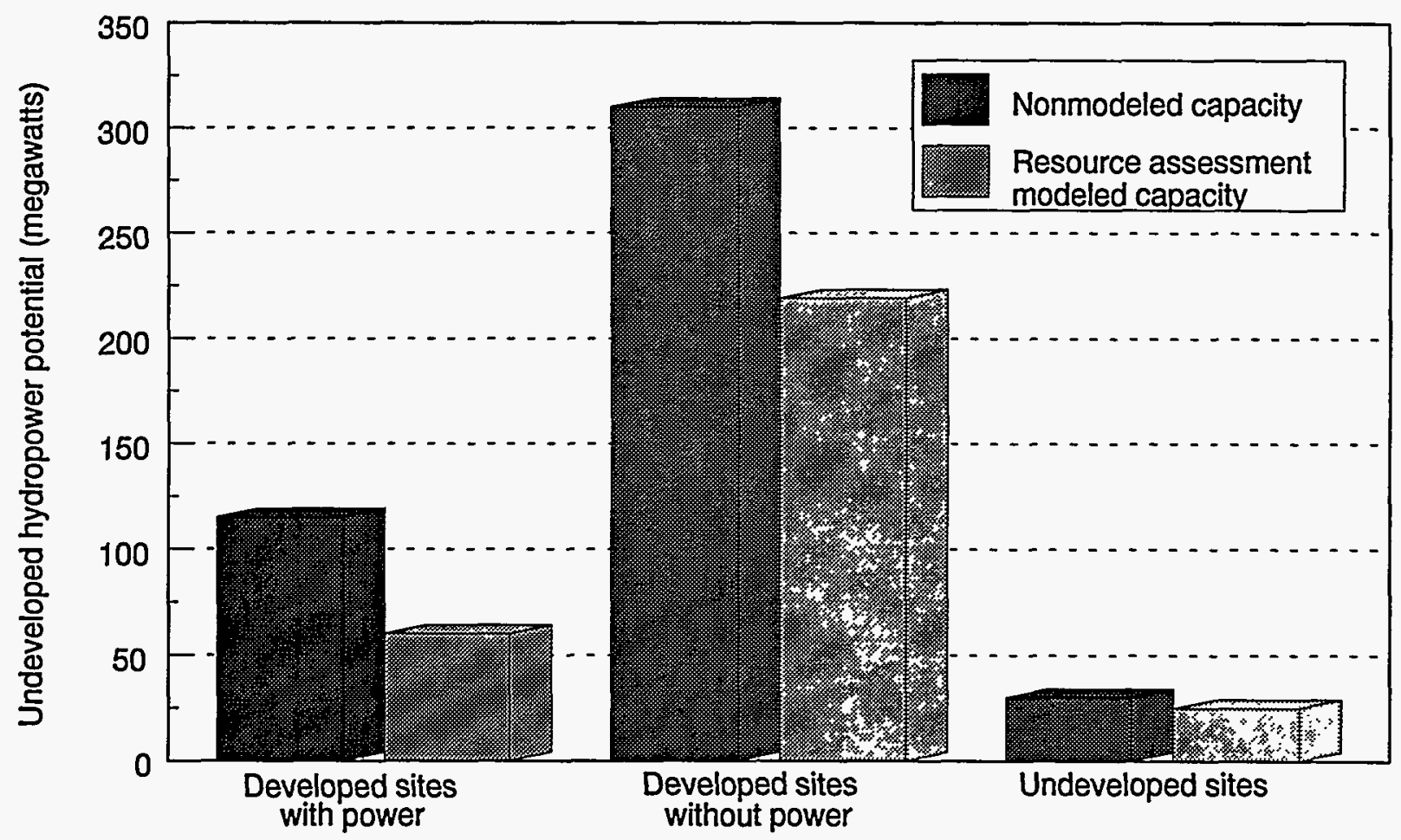

Figure 2. The HES-modeled undeveloped hydropower potential and the nonmodeled undeveloped hydropower potential. 


\section{Detailed Results}

The appendices contain, in the form of HESgenerated reports, detailed information of the undeveloped hydropower potential in Iowa. The appendices contain the following information:

Appendix A The undeveloped hydropower potential summary groups sites by dam status. The number of sites, nonmodeled undeveloped hydropower potential, and HESmodeled undeveloped hydropower potential is provided based on the dam status.

Appendix B The hydropower resource assessment by river basin includes the project number, project name, stream name, dam status, nonmodeled undeveloped hydropower potential, and the HES-modeled undeveloped hydropower potential for each of the individual sites.
Appendix C This is a listing of the project numbers, plant name, stream name, if a site is Federally owned, nonmodeled undeveloped hydropower potential, and HES-modeled undeveloped hydropower potential. The sites are grouped by dam status.

Appendix D This section contains a resource database listing for each of the 79 sites in Iowa. Information includes plant name, stream, state, county, river basin and owner names, project number, name plate and HES-modeled undeveloped hydropower potential, the unit and plant types, dam status, latitude, longitude, and the environmental factors that the HES uses to determine the project environmental suitability factor.

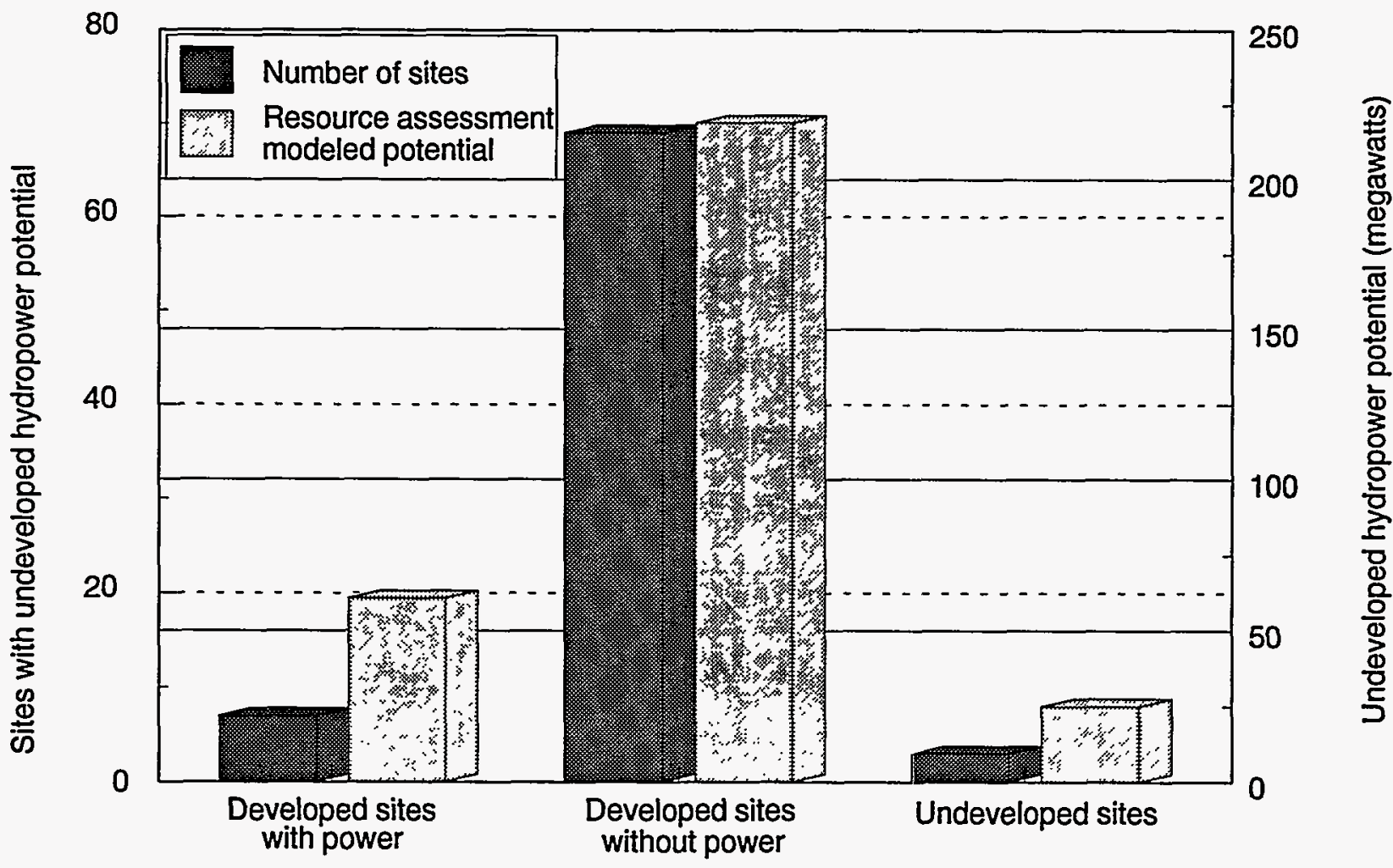

Figure 3. The HES-modeled undeveloped hydropower potential and the total megawatts of HESmodeled undeveloped hydropower potential. 


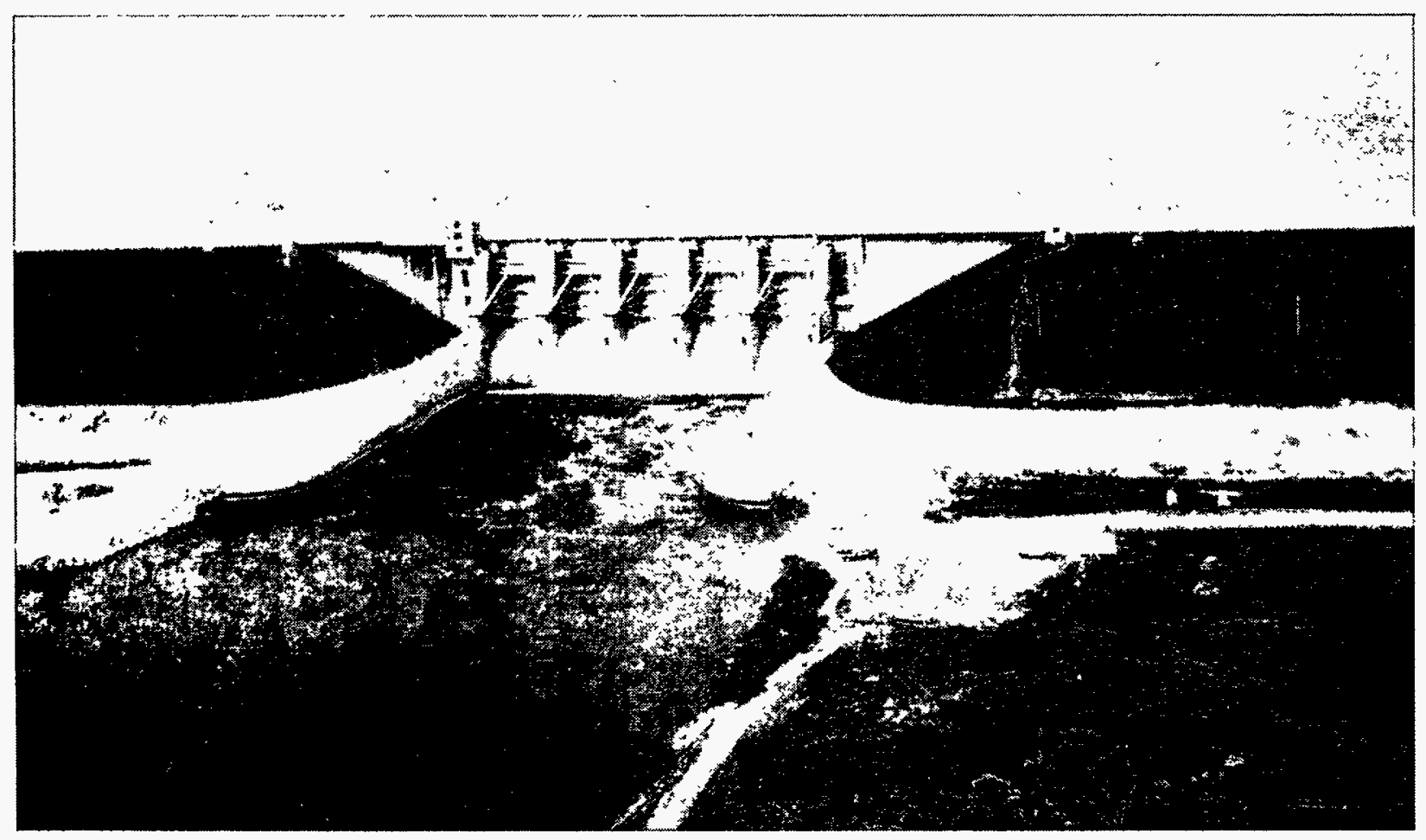

Figure 4. Example of a developed hydroelectric plant in Iowa. This is the Red Rock Lake, Iowa, hydroelectric plant.

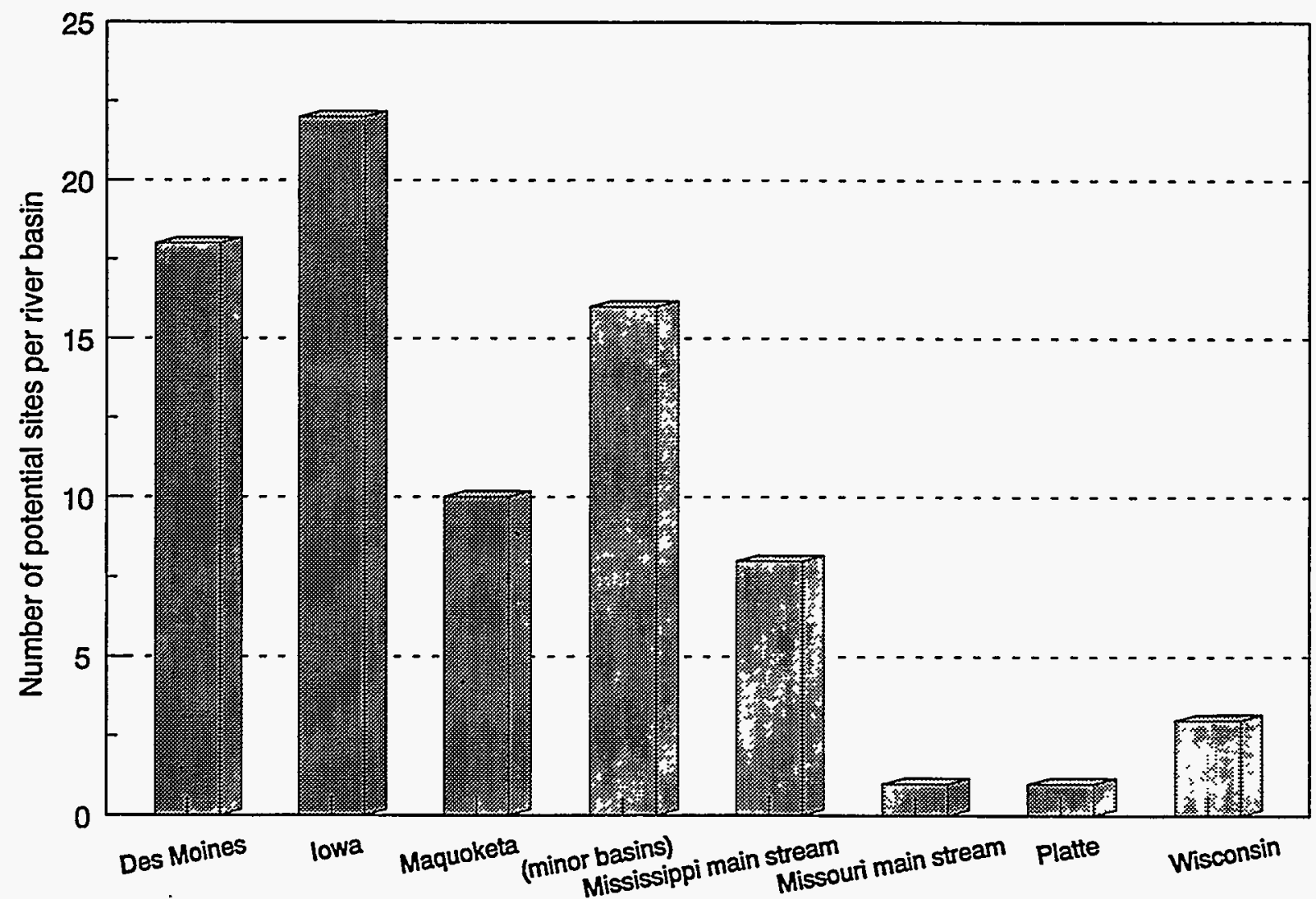

Figure 5. Number of sites with undeveloped hydropower potential in each of the Iowa river basins. 


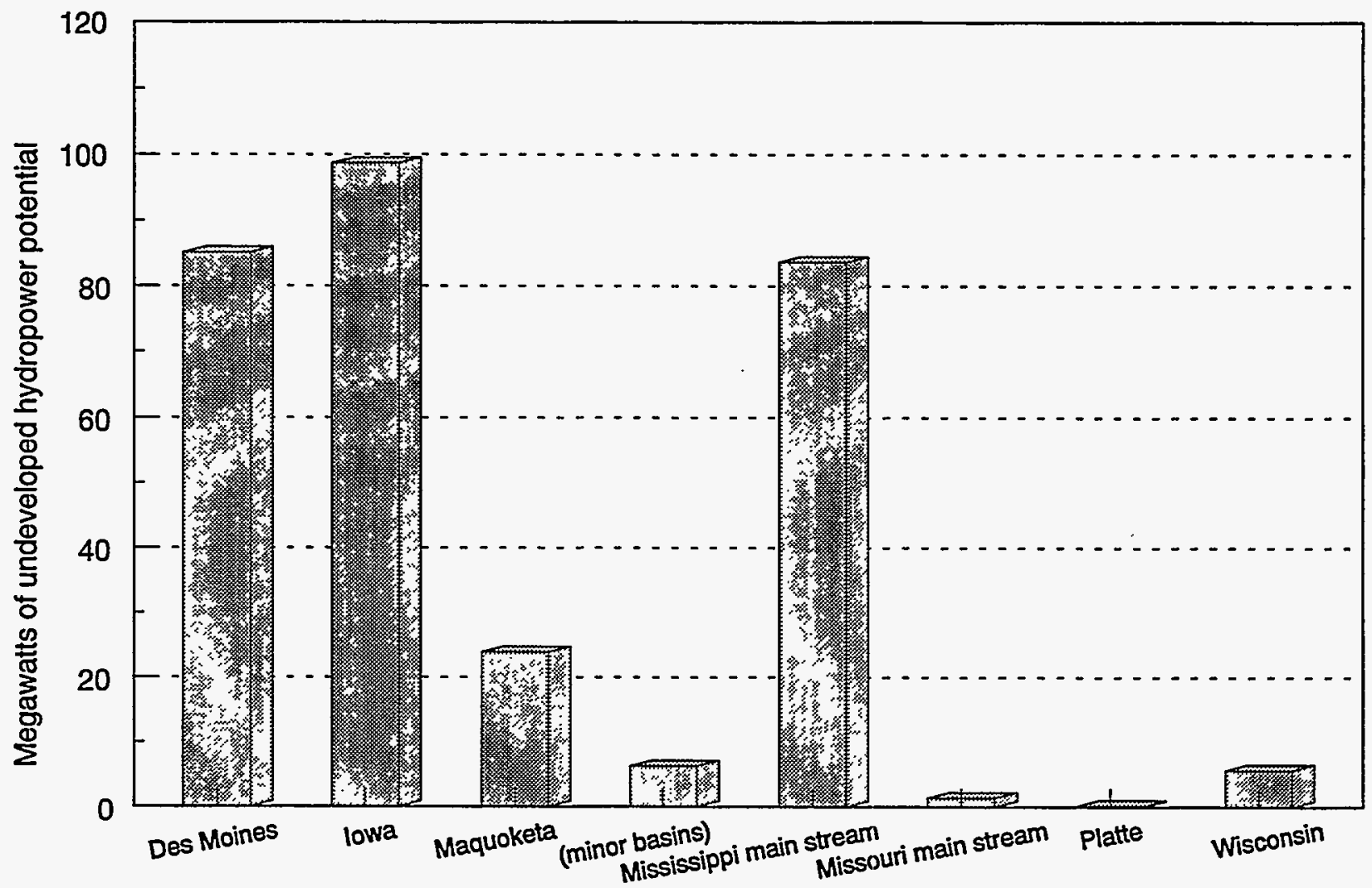

Figure 6. Megawatts of HES-modeled undeveloped hydropower potential in the Iowa river basins.

\section{OBTAINING INDIVIDUAL STATE INFORMATION}

Additional copies of the hydropower resource assessment results for individual states are available and can be obtained by writing or calling the National Technical Information Service (NTIS).

Telephone Orders - (703) 487-4650. NTIS sales desk and customer services are available between 8:30 a.m. and 5:00 p.m., Eastern Standard Time.

Fax - (703) 321-8547. Customers may fax their orders to NTIS. These orders may be charged to a NTIS deposit account, American Express, VISA, or MasterCard.

Mail Orders - Mail orders should be sent to National Technical Information Service, Document Sales, 5285 Port Royal Road, Springfield, VA 22161. Call the sales desk for prices before placing an order.
Method of Payment - Customers may pay for reports (and other NTIS products and services) by (a) credit card (American Express, Visa or MasterCard); (b) check or money order on a United States bank payable to NTIS; (c) a NTIS deposit account; or, (d) by asking to be billed (add $\$ 7.50$ per order), United States, Canada, and Mexico, only.

Handling Fee - A $\$ 3.00$ handling fee per total order applies to orders from the United States, Canada, and Mexico. Handling charges do not apply to rush order service or pick-up orders.

Postage and Shipping - Orders are shipped first class mail, or equivalent, to addresses in the United States, Canada, and Mexico.

Order Turnaround Time - Orders for technical reports generally are shipped within 2 to 8 days of receipt. For faster service, NTIS offers rush order service.

Rush Order Service - Call 1-800-533-NTIS. In Virginia, Canada, and Mexico call (703) 
487-4700. For NTIS rush order service add $\$ 15.00$ per item. This guarantees that an order will be processed through NTIS within 24 hours of its receipt. These orders receive immediate, individual attention. The items ordered are delivered by first call mail. Call NTIS for information on rush order service for computer products.

For Help in Tracing an Order - Call (703) 487-4650 and request the customer service option.

\section{ADDITIONAL HYDROPOWER EVALUATION SOFTWARE INFORMATION}

Additional information concerning the HES can be obtained by contacting Ben Rinehart or Jim Francfort at the addresses provided below. Copies of the software and the User's Manual may also be obtained from these individuals.

Ben Rinehart

Idaho National Engineering Laboratory

P.O. Box 1625, M.S. 3830

Idaho Falls, ID 83415-3830

(208) 526-1002

Jim Francfort

Idaho National Engineering Laboratory
P.O. Box 1625, M.S. 3875

Idaho Falls, ID 83415-3875

(208) 526-6787

Information concerning the State of Iowa's involvement with the resource assessment or about the identified sites may be obtained by contacting:

Ward Lenz

State Of Iowa

Iowa Department of Natural Resources

Wallace State Office Building

Des Moines, Iowa 50319

(515) 281-5145

\section{REFERENCES}

Francfort, J. E., S. D. Matthews, and B. N. Rinehart, 1991, Hydropower Evaluation Software User's Manual, DOE/ID-10338, Idaho National Engineering Laboratory, Idaho Falls, Idaho.

Francfort, J.E., K. M. Moore, and B. N. Rinehart, 1993, Uniform Criteria for U.S. Hydropower Resource Assessment, Hydropower Evaluation Software Status Report, DOE/ID-10430, Idaho National Engineering Laboratory, Idaho Falls, Idaho. 
$\mid$ 


\section{Appendix A \\ Summary Report}

A-1 


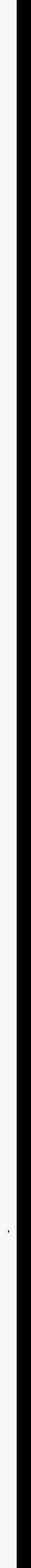




\begin{tabular}{|c|c|c|c|c|}
\hline State & Category & $\begin{array}{l}\text { Number of } \\
\text { Projects }\end{array}$ & $\begin{array}{l}\text { Name Plate } \\
\text { Capacity (KW) }\end{array}$ & $\begin{array}{l}\text { HES Adjusted } \\
\text { Capacity (KW) }\end{array}$ \\
\hline \multirow[t]{4}{*}{ IA } & With Power & 7 & 115451 & 60717.50 \\
\hline & พ/O Power & 69 & 310027 & 219103 \\
\hline & Undeveloped & 3 & 29980 & 25045 \\
\hline & STATE TOTAL & 79 & 455458 & 304865.50 \\
\hline \multirow[t]{3}{*}{ TOTALS } & with Power & 7 & 115451 & 60717.50 \\
\hline & w/O Power & 69 & 310027 & 219103 \\
\hline & Undeveloped & 3 & 29980 & 25045 \\
\hline \multicolumn{2}{|c|}{ GRAND TOTAL } & 79 & 455458 & 304865.50 \\
\hline
\end{tabular}




\section{Appendix B}

River Basins Report 

Page No.

$11 / 24 / 95$

$\begin{array}{cc}\text { FERC } & \text { Plant Name/ } \\ \text { Number } & \text { Stream }\end{array}$
Dam Name Plate
Stat Rating (KW) PESF PESF * KW

** River Basin DES MOINES
IA005 PANORA(232 IA NO)

M RACCOON $\mathbf{R}$

IA004 OTTUMWA

DES MOINES $R$

IA007 RED RंOCK

DES MOINES $R$

11502 RED ROCK

DES MOINES $R$

$\begin{array}{ll}11174 & \text { RED ROCK } \\ & \text { DES MOINES R }\end{array}$

09882 ADEL MILL

RACCOON R

08741 REDFIELD

$M$ RACCOON R

07879 CENTER STREET

DES MOINES $R$

10749 SAYLORVILLE

DES MOINES $R$

11346 FORT DODGE MILL

DES MOINES $R$

11344 HUMBOLDT MILL

$W$ FK DES MOINES $R$

11343 RUTLAND MILL

W FK DES MOINES $R$

IA101 FRASER MILLDAM

DES MOINES RIVER

IA102 BOONE MILIDAM

DES MOINES RIVER

IA103 SCOTT STREET DAM

DES MOINES RIVER

IA109 BONAPARTE

DES MOINES RIVER
U

W

พั

พอ

พอ

พอ

พอ

พอ

พอ

พอ

พั

พอ

พอ

พo

พอ

$420.00 \quad 0.90$

378.00

$98.00 \quad 0.50$

49.00

no

$1860.00 \quad 0.90$

1674.00

$9600.00 \quad 0.75$

7200.00

621.00

$690.00 \quad 0.90$

$820.00 \quad 0.90$

738.00

630.00

$700.00 \quad 0.90$

$760.00 \quad 0.90$

684.00

684.00

$760.00 \quad 0.90$

$1470.00 \quad 0.90$

1323.00

พั
$2990.00 \quad 0.90$

2691.00 


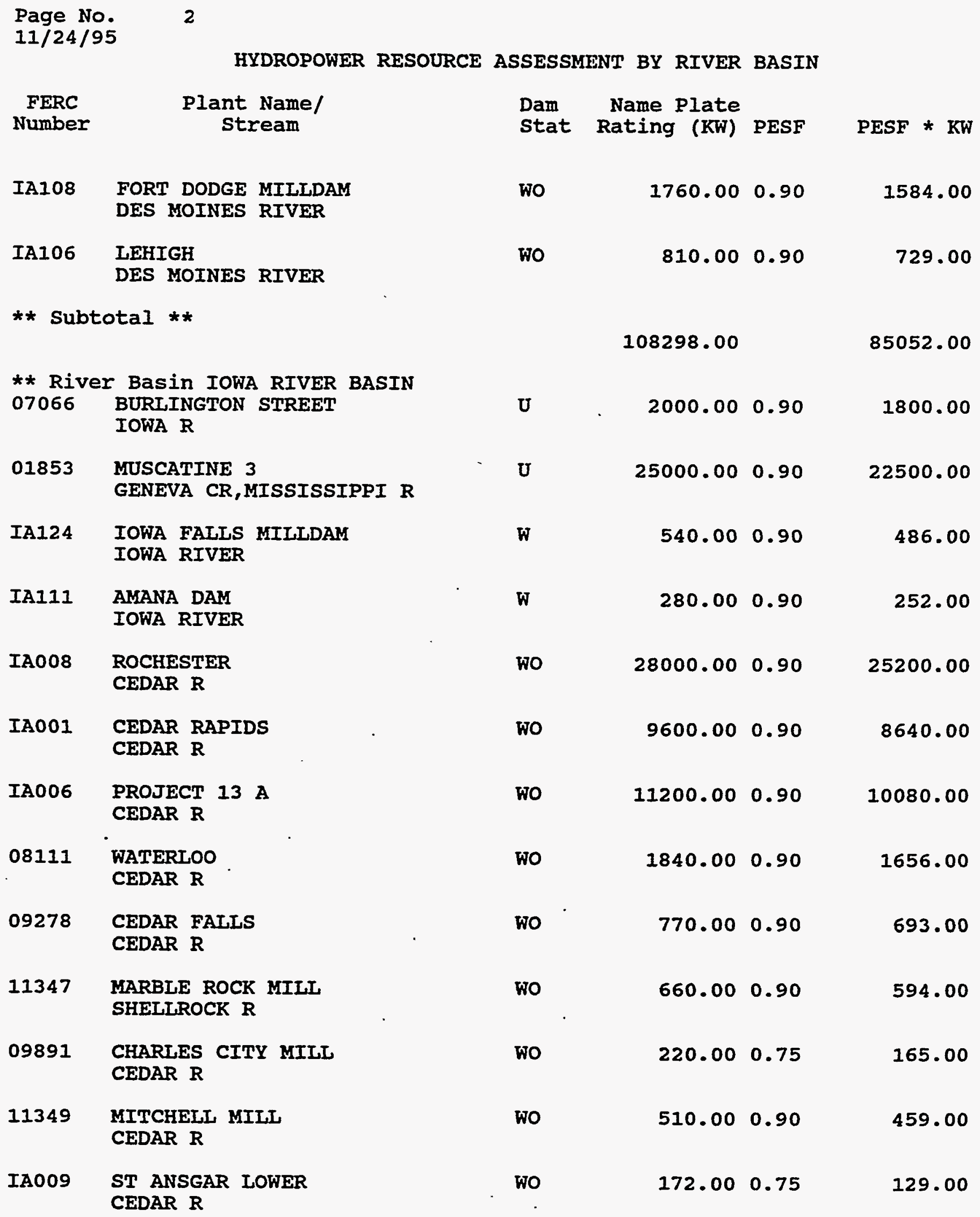


Page No.

$11 / 24 / 95$

$\begin{array}{cc}\text { FERC } & \text { Plant Name/ } \\ \text { Number } & \text { Stream }\end{array}$

IA010 ST ANSGAR UPPER CEDAR R

08691 CORALVILLE MILL IOWA $\mathrm{R}$

08109 STEAMBOAT ROCK IOWA $R$

08110 ALDEN MILL

IOWA $\mathbf{R}$

07103 MISSISSIPPI L\&D 17 MISSISSIPPI $R$

11250 MISSISSIPPI I\&D 16 MISSISSIPPI $R$

11463 CORALVILLE IOWA $\mathbf{R}$

11283 NASHUA CEDAR R

11374 GREENE MILL SHELLROCK R
Dam Name Plate

stat Rating (KW) PESF PESF * KW

พo

$$
186.00 \quad 0.75
$$

139.50

พั

$$
1000.00 \quad 0.75
$$

750.00

พอ

$$
280.00 \quad 0.50
$$

140.00

พอ

$$
220.00 \quad 0.50
$$

110.00

พั

$10500.00 \quad 0.50$

5250.00

พอ

$13000.00 \quad 0.75$

9750.00

พอ

$12000.00 \quad 0.75$

9000.00

พั

พอ

$810.00 \quad 0.75$

607.50

333.00

$370.00 \quad 0.90$

$$
333.00
$$

119158.00

98734.00

** River Basin MAQUOKETA RIVER BASIN

\section{IA002 MAQUOKETA \\ $S$ FK MAQUOKETA $R$}

พ

$4140.00 \quad 0.50$

2070.00

11342 MONTICELLO MILI

MAQUOKETA $\mathrm{R}$

พั

$320.00 \quad 0.90$

288.00

09892 MANCHESTER MILI

MAQUOKETA $\mathbf{R}$

พอ

$150.00 \quad 0.75$

112.50

พo

$250.00 \quad 0.90$

225.00

MAQUOKETA $R$

11485 DELHI MILL DAM
MAQUOKETA $R$

พอ

พอ

$1425.00 \quad 0.90$

1282.50

08184 QUAKER MILLS

MAQUOKETA $R$
$160.00 \quad 0.90$

144.00 


\begin{tabular}{|c|c|c|c|c|c|}
\hline & HYDROPOWER RESOURCE & ASSESSN & IENT BY RIVER & BASIN & \\
\hline $\begin{array}{l}\text { FERC } \\
\text { Number }\end{array}$ & $\begin{array}{c}\text { Plant Name/ } \\
\text { Stream }\end{array}$ & $\begin{array}{l}\text { Dam } \\
\text { stat }\end{array}$ & $\begin{array}{l}\text { Name Plate } \\
\text { Rating (KW) }\end{array}$ & PESF & PESF * KW \\
\hline 11248 & $\begin{array}{ll}\text { MISSISSIPPI } & \text { L\&D } 12 \\
\text { MISSISSIPPI } & R\end{array}$ & พO & 8200.00 & 0.50 & 4100.00 \\
\hline 11247 & $\begin{array}{l}\text { MISSISSIPPI L\&D } 11 \\
\text { MISSISSIPPI } \mathrm{R}\end{array}$ & Wo & 14000.00 & 0.50 & 7000.00 \\
\hline 08642 & $\begin{array}{l}\text { ELRADER } \\
\text { TURKEY R }\end{array}$ & พอ & 500.00 & 0.50 & 250.00 \\
\hline 11246 & $\begin{array}{l}\text { MISSISSIPPI I\&D } 10 \\
\text { MISSISSIPPI } R\end{array}$ & พั & 16800.00 & 0.50 & 8400.00 \\
\hline \multicolumn{6}{|c|}{ ** Subtotal ** } \\
\hline $\begin{array}{l}\text { ** Rive } \\
\text { IA117 }\end{array}$ & $\begin{array}{l}\text { Basin MINOR RIVER BASIN } \\
\text { WAVERLY MILLDAM } \\
\text { CEDAR RIVER }\end{array}$ & $\mathbf{w}$ & 580.00 & 0.90 & 522.00 \\
\hline IA113 & $\begin{array}{l}\text { ANAMOSA MILLDAM } \\
\text { WAPSIPINICON RIVER }\end{array}$ & $\mathbf{W}$ & 700.00 & 0.90 & 630.00 \\
\hline IA118 & $\begin{array}{l}\text { QUASQUETON DAM } \\
\text { WAPSIPINICON RIVER }\end{array}$ & พั & 250.00 & 0.90 & 225.00 \\
\hline IA119 & $\begin{array}{l}\text { MASON CITY MILLDAM } \\
\text { WINNEBAGO RIVER }\end{array}$ & พO & 100.00 & 0.90 & 90.00 \\
\hline IA122 & $\begin{array}{l}\text { CLERMONT MILLDAM } \\
\text { TURKEY RIVER }\end{array}$ & พO & 470.00 & 0.90 & 423.00 \\
\hline IA107 & $\begin{array}{l}\text { ROCKFORD } \\
\text { SHELL ROCK RIVER }\end{array}$ & พо & 150.00 & 0.90 & 135.00 \\
\hline IA110 & $\begin{array}{l}\text { LITTLE SIOUX DAM } \\
\text { LITTLE SIOUX RIVER }\end{array}$ & พ० & 690.00 & 0.90 & 621.00 \\
\hline IA112 & $\begin{array}{l}\text { LYNNVILLE DAM } \\
\text { NORTH SKUNK RIVER }\end{array}$ & พO & 150.00 & 0.90 & 135.00 \\
\hline IA114 & $\begin{array}{l}\text { OXFORD MILLS } \\
\text { WAPSIPINICON RIVER }\end{array}$ & พั & 570.00 & 0.90 & 513.00 \\
\hline IA115 & $\begin{array}{l}\text { PAIISADES-KEPLER } \\
\text { CEDAR RIVER }\end{array}$ & พั & 2010.00 & 0.90 & 1809.00 \\
\hline IA116 & $\begin{array}{l}\text { TROY MILLS } \\
\text { WAPSIPINICON RIVER }\end{array}$ & พั & 330.00 & 0.90 & 297.00 \\
\hline
\end{tabular}


Page No.

$11 / 24 / 95$

$\begin{array}{cc}\text { FERC } & \text { Plant Name/ } \\ \text { Number } & \text { Stream }\end{array}$

IA121 KLONDIKE MILLDAM

BIG SIOUX RIVER

IA123 OTRANTO DAM

CEDAR RIVER

IA125 CLARINDA DAM

NODAWAY RIVER

IA105 FORT ATKINSON

TURKEY RIVER

IA104 FERTILE MILIDAM

WINNEBAGO RIVER
Dam Name Plate

stat Rating (KW) PESF PESF * KW

พั

$440.00 \quad 0.90$

396.00

พอ

$150.00 \quad 0.90$

135.00

Wo

140.000 .90

126.00

พอ

$100.00 \quad 0.90$

90.00

พั

$130.00 \quad 0.90$

117.00

$$
6960.00
$$

6264.00

** River Basin MISSISSIPPI MAIN STREAM

IA003 MISSISSIPPI L\&D 19

MISSISSIPPI $R$

พ

103831.000 .50

51915.50

พั

$1060.00 \quad 0.50$

530.00

SKUNK $R$

11251 MISSISSIPPI L\&D 18. MISSISSIPPI $R$

11278 L\&D 15

MISSISSIPPI R

08105 CENTRAL CITY MILL

WAPSIPINICON $\mathrm{R}$

10229 INDEPENDENCE MILL

WAPSIPINICON $\mathbf{R}$

08098 IITTLETON DAM

WAPSIPINICON $\mathbf{R}$

11249 MISSISSIPPI L\&D 13

MISSISSIPPI $R$

** Subtotal **

** River Basin MISSOURI MAIN STREAM

10747 RATHBUN DAM CHARITON $\mathrm{R}$
พั

$11000.00 \quad 0.75$

8250.00

พo

พั

พั

พั

พั

$$
29000.00 \quad 0.50
$$

14500.00

$640.00 \quad 0.50$

320.00

$706.00 \quad 0.50$

353.00

160.00

$320.00 \quad 0.50$

$15200.00 \quad 0.50$

7600.00

161757.00

83628.50

พั

$1910.00 \quad 0.75$

1432.50 
Page No. 6

$11 / 24 / 95$

HYDROPOWER RESOURCE ASSESSMJENT BY RIVER BASIN

FERC

Number
Plant Name/

stream

Dam Name Plate

stat Rating (KW) PESF PESF * KW

1432.50

พั

$310.00 \quad 0.50$

$$
310.00
$$

155.00
08097 DECORAH UPPER
UPPER IOWA R

08097 DECORAH UPPER
UPPER IOWA R

\section{MISSISSIPPI $L \& D$
MISSISSIPPI $\mathbf{R}$}

09327 DECORAH LOWER

UPPER IOWA $R$

** Subtotal **

*** Total ***
พั

พอ

พอ

N RIVER BASIN 9

$10000.00 \quad 0.50$

5000.00

502.50

225.00

11120.00

5727.50

455458.00

304865.50 
Appendix C

lowa Sites Listing 
HYDROPOWER RESOURCE ASSESSMENT BY FERC NUMBER

\section{Plant Name/} stream

\section{$\mathbf{s}$}

Dam

ST stat

Name Plate

Rating (KW) PESF

IA U

$25000.00 \quad 0.90$

MUSCATINE 3

GENEVA CR,MISSISSIPPI $R$

** Subtotal **

** FERC Number 07066

BURLINGTON STREET

IOWA $\mathbf{R}$

** Subtotal

** FERC Number IA005

PANORA (232 IA NO)

M RACCOON R

** Subtotal **

** FERC Number IA002

MAQUOKETA

$S$ FK MAQUOKETA $R$

** Subtotal **

** FERC Number IA003

MISSISSIPPI L\&D 19

MISSISSIPPI $\mathbf{R}$

** Subtotal **

** FERC Number IA004

OTTUMWA

DES MOINES $R$

** Subtotal **

** FERC Number IA111

AMANA DAM

IOWA RIVER
IA $W$

103831.00

4140.00

2070.00

IA W

$103831.00 \quad 0.50$

51915.50

IA U

$$
2980.00
$$

745.00

2070.00

51915.50

4842.00

5380.00

4842.00

IA $W$

$280.00 \quad 0.90$

252.00 


\section{Plant Name/ stream}

** Subtotal **

** FERC Number IA113

ANAMOSA MILILDAM

WAPSIPINICON RIVER

** Subtotal **

** FERC Number IA117

WAVERIY MILIDAM

CEDAR RIVER

** Subtotal **

** FERC Number IA124

IOWA FALIS MILLDAM

IOWA RIVER

** Subtotal **

** FERC Number 07103

MISSISSIPPI I\&D 17

MISSISSIPPI $R$

** Subtotal **

** FERC Number 07879

CENTER STREET

DES MOINES $R$

** Subtotal **

** FERC Number 08096

IINN GROVE MILL

LITTLE SIOUX $R$

** Subtotal ** $\begin{array}{lll}\text { Dam } & \text { Name Plate } \\ \text { Stat } & \text { Rating (KW) PESF } \quad \text { PESF * KW }\end{array}$

IA W

$$
280.00
$$

252.00

630.00

700.00

630.00

IA $W$

$580.00 \quad 0.90$

522.00

580.00

522.00

IA $W$

$540.00 \quad 0.90$

486.00

540.00

486.00

IA พั

$10500.00 \quad 0.50$

5250.00

10500.00

5250.00

IA พо

$1860.00 \quad 0.90$

1674.00

1860.00

1674.00

IA พั

$310.00 \quad 0.50$

155.00

310.00

155.00 


\section{Plant Name/ Stream}

** FERC Number 08097 DECORAH UPPER UPPER IOWA $R$

** Subtotal **

** FERC Number 08098 IITTLETON DAM WAPSIPINICON $\mathbf{R}$

** Subtotal **

** FERC Number 08105 CENTRAL CITY MILL WAPSIPINICON $\mathbf{R}$

** Subtotal **

** FERC Number 08109 STEAMBOAT ROCK IOWA $\mathbf{R}$

** Subtotal **

** FERC Number 08110 AIDEN MILL

IOWA $\mathbf{R}$

** Subtotal **

** FERC Number 08111 WATERLOO CEDAR $R$

** Subtotal **

** FERC Number 08184 QUAKER MILLS MAQUOKETA R

$\begin{array}{lll}\text { Dam } & \text { Name Plate } & \\ \text { ST Stat Rating (KW) PESF } & \text { PESF * } \mathrm{KW}\end{array}$

IA พo

$$
450.00 \quad 0.50
$$

225.00

$$
450.00
$$

225.00

IA wo

$320.00 \quad 0.50$

160.00

$$
320.00
$$

160.00

IA WO

$640.00 \quad 0.50$

320.00

640.00

320.00

IA พO

$280.00 \quad 0.50$

140.00

280.00

140.00

IA พั

$220.00 \quad 0.50$

110.00

220.00

110.00

IA พ०

$1840.00 \quad 0.90$

1656.00

1840.00

1656.00

IA พั

$160.00 \quad 0.90$

144.00 
Page No. 4

$11 / 24 / 95$

HYDROPOWER RESOURCE ASSESSMINT BY FERC NUMBER

Plant Name/

Stream

** Subtotal **

** FERC Number 08642

ELKADER

TURKEY $R$

** Subtotal **

** FERC Number 08691

CORALVILLE MILI

IOWA $\mathbf{R}$

** Subtotal **

** FERC Number 08741

REDFIELD

M RACCOON $\mathbf{R}$

** Subtotal **

** FERC Number 08955

OAKLAND MILLS

SKUNK $R$

** Subtotal **

** FERC Number 09278

CEDAR FALLS

CEDAR R

** Subtotal **

** FERC Number 09327

DECORAH LOWER

UPPER IOWA $\mathbf{R}$

** Subtotal ** $\begin{array}{lll}\text { Dam Name Plate } & \text { Nam } \\ \text { Stat } & \text { Rating (KW) PESF * KW }\end{array}$

$$
160.00
$$

144.00

IA พO

$$
500.00 \quad 0.50
$$

250.00

$$
500.00
$$

250.00

IA Wo

$1000.00 \quad 0.75$

750.00

$$
1000.00
$$

750.00

IA Wo

$$
98.00 \quad 0.50
$$

49.00

98.00

49.00

IA Wo

$1060.00 \quad 0.50$

530.00

1060.00

530.00

IA Wo

$770.00 \quad 0.90$

693.00

770.00

693.00

IA พ०

$670.00 \quad 0.75$

502.50

670.00

502.50 
Page No.

5

$11 / 24 / 95$

HYDROPOWER RESOURCE ASSESSMENT BY FERC NUMBER

Plant Name/ Stream

** FERC Number 09882

ADEL MILI

RACCOON R

** subtotal

** FERC Number 09891

CHARLES CITY MILL

CEDAR R

** Subtótal

** FERC Number 09892

MANCHESTER MILL

MAQUOKETA $R$

** Subtotal **

** FERC Number 09893

HOPKINTON DAM

MAQUOKETA $R$

** Subtotal **

** FERC Number 10229

INDEPENDENCE MIII

WAPSIPINICON $R$

** Subtotal **

** FERC Number 10747

RATHBUN DAM

CHARITON $\mathbf{R}$

** Subtotal **

** FERC Number 10749

SAYLORVILLE

DES MOINES $R$

$\begin{array}{lll}\text { Dam } & \text { Name Plate } & \\ \text { ST Stat } & \text { Rating (KW) PESF } & \text { PESF * KW }\end{array}$

IA Wo

$$
420.00 \quad 0.90
$$

378.00

420.00

378.00

IA พO

$220.00 \quad 0.75$

165.00

220.00

165.00

IA พอ

$150.00 \quad 0.75$

112.50

150.00

112.50

IA พั

$250.00 \quad 0.90$

225.00

250.00

225.00

IA พO

$706.00 \quad 0.50$

353.00

706.00

353.00

IA พั

$1910.00 \quad 0.75$

1432.50

1910.00

1432.50

IA พO

$9600.00 \quad 0.75$

7200.00 
Plant Name/ Stream

** Subtotal **

** FERC Number 11174

RED ROCK

DES MOINES $R$.

** Subtotal **

** FERC Number 11245

MISSISSIPPI I\&D 9

MISSISSIPPI $R$

** Subtotal **

** FERC Number 11246

MISSISSIPPI I\&D 10

MISSISSIPPI $R$

** Subtotal **

** FERC Number 11247

MISSISSIPPI L\&D 11

MISSISSIPPI $R$

** Subtotal **

** FERC Number 11248

MISSISSIPPI L\&D 12

MISSISSIPPI $\mathrm{R}$.

** Subtotal **

** FERC Number 11249

MISSISSIPPI L\&D 13

MISSISSIPPI $R$

** Subtotal ** $\begin{array}{lll}\text { Dam } & \text { Name Plate } & \\ \text { ST Stat } & \text { Rating (KW) PESF } & \text { PESF * KW }\end{array}$

IA พั

$30000.00 \quad 0.75$

22500.00

30000.00

22500.00

IA พO

$10000.00 \quad 0.50$

5000.00

10000.00

5000.00

IA พO

$16800.00 \quad 0.50$

8400.00

16800.00

8400.00

IA พO

$14000.00 \quad 0.50$

7000.00

14000.00

7000.00

IA พo

$$
8200.00 \quad 0.50
$$

4100.00

8200.00

4100.00

IA พO

$15200.00 \quad 0.50$

7600.00

15200.00

7600.00 
Plant Name/

Stream

** FERC Number 11250

MISSISSIPPI L\&D 16

MISSISSIPPI $R$

** Subtotal **

** FERC Number 11251

MISSISSIPPI L\&D 18

MISSISSIPPI $R$

** Subtotal $\star *$

** FERC Number 11278

I\&D 15

MISSISSIPPI R

** Subtotal **

** FERC Number 11283

NASHUA

CEDAR R

** Subtotal

** FERC Number 11342

MONTICELLO MILL

MAQUOKETA $\mathrm{R}$

** Subtotal **

** FERC Number 11343

RUTLAND MILI

W FK DES MOINES R

** Subtotal **

** FERC Number 11344

HUMBOLDT MILL

$W$ FK DES MOINES $R$

Dam
STame Plate
Stat
Rating (KW) PESF $\quad$ PESF * $\mathrm{KW}$

IA พั

$13000.00 \quad 0.75$

9750.00

13000.00

9750.00

IA Wo

$11000.00 \quad 0.75$

8250.00

11000.00

8250.00

IA พั

$29000.00 \quad 0.50$

14500.00

29000.00

14500.00

IA พอ

810.000 .75

607.50

810.00

607.50

IA พั

$320.00 \quad 0.90$

288.00

320.00

288.00

IA พั

$700.00 \quad 0.90$

630.00

700.00

630.00

IA พอ

$820.00 \quad 0.90$

738.00 
Plant Name/

Stream

** Subtotal **

** FERC Number 11346

FORT DODGE MILL

DES MOINES $R$

** Subtotal **

** FERC Number 11347

MARBLE ROCK MILI

SHELIROCK $R$

** Subtotal **

** FERC Number 11349

MITCHELI MIIL

CEDAR $R$

** Subtotal **

** FERC Number 11374

GREENE MIIL

SHELLROCK $R$

** Subtotal **

** FERC Number 11463

CORAIVILLE

IOWA $\mathbf{R}$

** Subtotal **

** FERC Number 11485

DELHI MILI DAM

MAQUOKETA $R$

** Subtotal ** $\begin{array}{lll}\text { Dam } & \text { Name Plate } & \\ \text { ST Stat } & \text { Rating (KW) PESF } & \text { PESF * KW }\end{array}$

$$
820.00
$$

738.00

IA พO .

690.000 .90

621.00

690.00

621.00

IA Wo

$660.00 \quad 0.90$

594.00

660.00

594.00

IA พO

$510.00 \quad 0.90$

459.00

510.00

459.00

IA พั

$370.00 \quad 0.90$

333.00

370.00

333.00

IA wo

$12000.00 \quad 0.75$

9000.00

12000.00

9000.00

IA Wo

$1425.00 \quad 0.90$

1282.50

1425.00

1282.50 
HYDROPOWER RESOURCE ASSESSMENT BY FERC NUMBER

\section{Plant Name/} stream

** FERC Number 11502

RED ROCK

DES MOINES $R$

** Subtotal **

** FERC Number IAO01

CEDAR RAPIDS

CEDAR R

** Subtotal

** FERC Number IA006

PROJECT $13 \mathrm{~A}$

CEDAR R

** Subtotal **

** FERC Number IA007

RED ROCK

DES MOINES $R$

** Subtotal **

** FERC Number IA008 ROCHESTER

CEDAR R .

** Subtotal **

** FERC Number IA009

ST ANSGAR LOWER

CEDAR R

** Subtotal **

** FERC Number IAOIO

ST ANSGAR UPPER

CEDAR R

\section{Dam Name Plate \\ ST Stat Rating (KW) PESF \\ PESF * KW}

IA พอ

$$
30000.00 \quad 0.75
$$

22500.00

$$
30000.00
$$

22500.00

IA พอ

$$
9600.000 .90
$$

8640.00

$$
9600.00
$$

$$
8640.00
$$

IA พO

$11200.00 \quad 0.90$

10080.00

11200.00

10080.00

IA พO

17200.00

0.90

15480.00

17200.00

15480.00

IA พั

$28000.00 \quad 0.90$

25200.00

28000.00

25200.00

IA พ०

$$
172.00 \quad 0.75
$$

129.00

172.00

129.00

IA พั

139.50 
Page No. 10

$11 / 24 / 95$

HYDROPOWER RESOURCE ASSESSMENT BY FERC NUMBER

Plant Name/ Stream

** Subtotal **

** FERC Number IA101

FRASER MILIDAM

DES MOINES RIVER

** Subtotal **

** FERC Number IA102

BOONE MILLDAM

DES MOINES RIVER

** Subtotal **

** FERC Number IAI03

SCOTT STREET DAM

DES MOINES RIVER

** Subtotal **

** FERC Number IA104

FERTILE MILLDAM

WINNEBAGO RIVER

** Subtotal **

** FERC Number IA105

FORT ATKINSON

TURKEY RIVER

** Subtotal **

** FERC Number IA106

LEHIGH

DES MOINES RIVER

IA พO

IA พั

Dam ST

Name Plate

Rating (KW) PESF

186.00

139.50

IA พO

$760.00 \quad 0.90$

684.00

IA พO

$760.00 \quad 0.90$

684.00

760.00

684.00

IA พO

$1470.00 \quad 0.90$

1323.00

1470.00

1323.00

IA พั

$130.00 \quad 0.90$

117.00

130.00

117.00

$100.00 \quad 0.90$

90.00

100.00

90.00

** Subtota1 **
810.00

729.00 


\section{Plant Name/ \\ Stream}

** FERC Number IA107 ROCKFORD

SHELL ROCK RIVER

** Subtotal **

** FERC Number IA108 FORT DODGE MILLDAM DES MOINES RIVER

** Subtotal **

** FERC Number IA109 BONAPARTE DES MOINES RIVER

** Subtotal **

** FERC Number IA110

LITTLE SIOUX DAM

LITTLE SIOUX RIVER

** Subtotal **

** FERC Number IA112

LYNNVILLE DAM

NORTH SKUNK RIVER

** Subtotal

** FERC Number IA114

OXFORD MILLS

WAPSIPINICON RIVER

** Subtotal

** FERC Number IA115

PALISADES-KEPLER

CEDAR RIVER

$\begin{array}{lll}\text { Dam } & \text { Name Plate } & \\ \text { ST Stat } & \text { Rating (KW) PESF } & \text { PESF * KW }\end{array}$

IA พO

$150.00 \quad 0.90$

135.00

150.00

135.00

IA พั

$1760.00 \quad 0.90$

1584.00

1760.00

1584.00

IA พO

2990.00

0.90

2691.00

2990.00

2691.00

IA พO

$690.00 \quad 0.90$

621.00

690.00

621.00

IA พO

$150.00 \quad 0.90$

135.00

150.00

135.00

IA พO

$570.00 \quad 0.90$

513.00

570.00

513.00

IA พo

$2010.00 \quad 0.90$

1809.00 
Page No.

HYDROPOWER RESOURCE ASSESSHENT BY FERC NUMBER

Plant Name/

Stream $\begin{array}{ll}\text { Dam } & \text { Name Plate } \\ \text { ST Stat } & \text { Rating (KW) PESF }\end{array}$

2010.00

IA WO

TROY MILIS

WAPSIPINICON RIVER

** Subtotal **

** FERC Number IA118

QUASQUETON DAM

WAPSIPINICON RIVER

** Subtotal **

** FERC Number IA119

MASON CITY MIILLDAM

WINNEBAGO RIVER

** Subtotal **

** FERC Number IA121

KLONDIKE MILIDAM

BIG SIOUX RIVER

** Subtotal **

** FERC Number IA122

CLERMONT MILLDAM

TURKEY RIVER

** Subtotal **

** FERC Number IA123

OTRANTO DAM

CEDAR RIVER

** Subtotal **
IA พO

IA พั

IA พO

440.00

$470.00 \quad 0.90$

470.00

423.00

IA WO

150.00

0.90

135.00

150.00

135.00 
HYDROPOWER RESOURCE ASSESSMENT BY FERC NUMBER

Plant Name/

Stream

** FERC Number IA125

CLARINDA DAM

NODAWAY RIVER

** Subtotal **

*** Total ***

$\begin{array}{ll}\text { Dam } & \text { Name Plate } \\ \text { ST } & \text { Rating (KW) PESF } \quad \text { PESF * KW }\end{array}$

IA พั

$140.00 \quad 0.90$

126.00

140.00

126.00

455458.00

304865.50 



\section{Appendix D}

Individual Resource Database Listing 


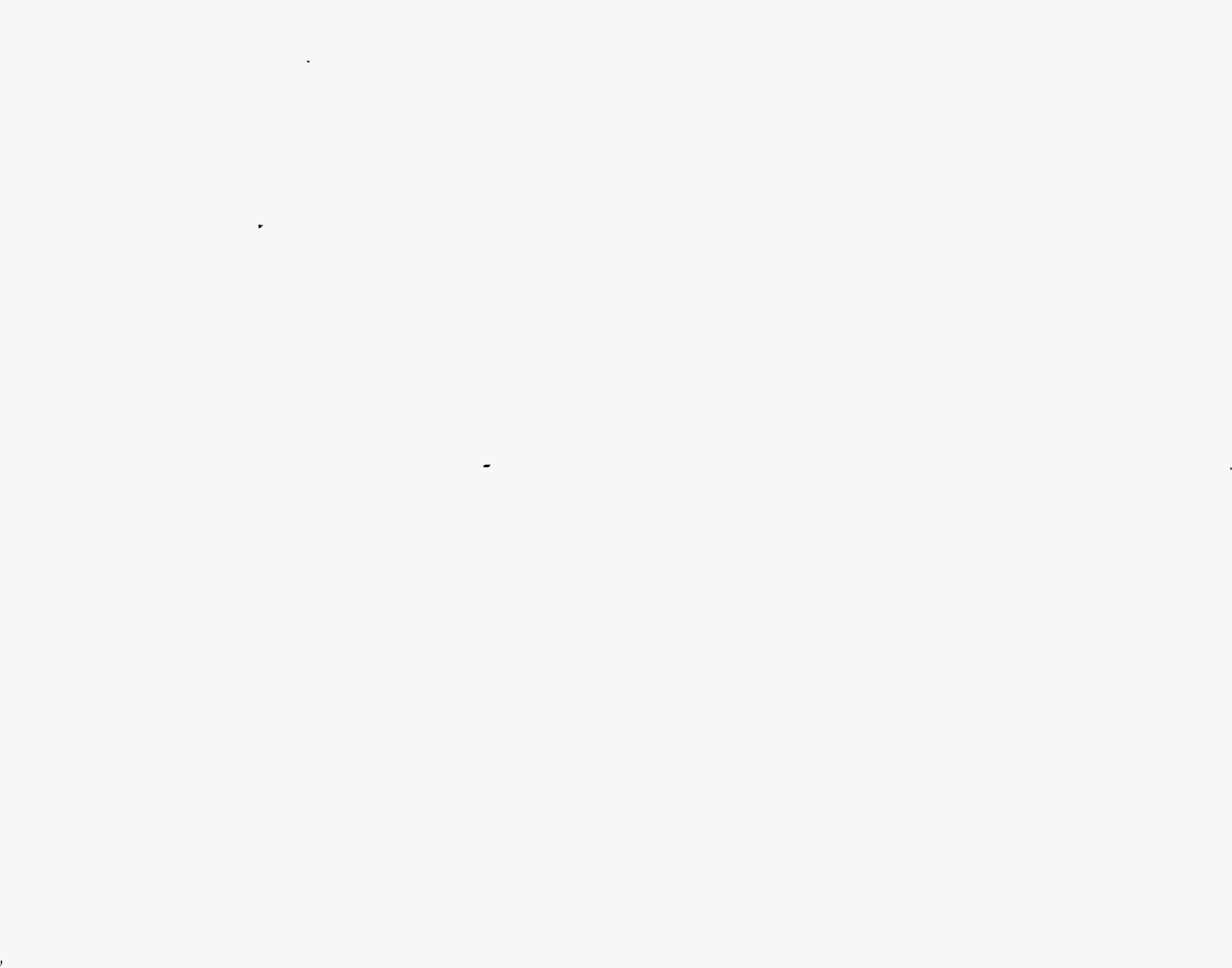


DATE: $11 / 24 / 95$

PAGE NO: 1

FERC

Number

01853 MUSCATINE 3

county Name

MUSCATINE
Plant Name

C FIRST IOWA HYDRO EL COOP

River Basin

IOWA RIVER BASIN state

Name

IA

GENEVA CR,MISSISSIPPI $R$

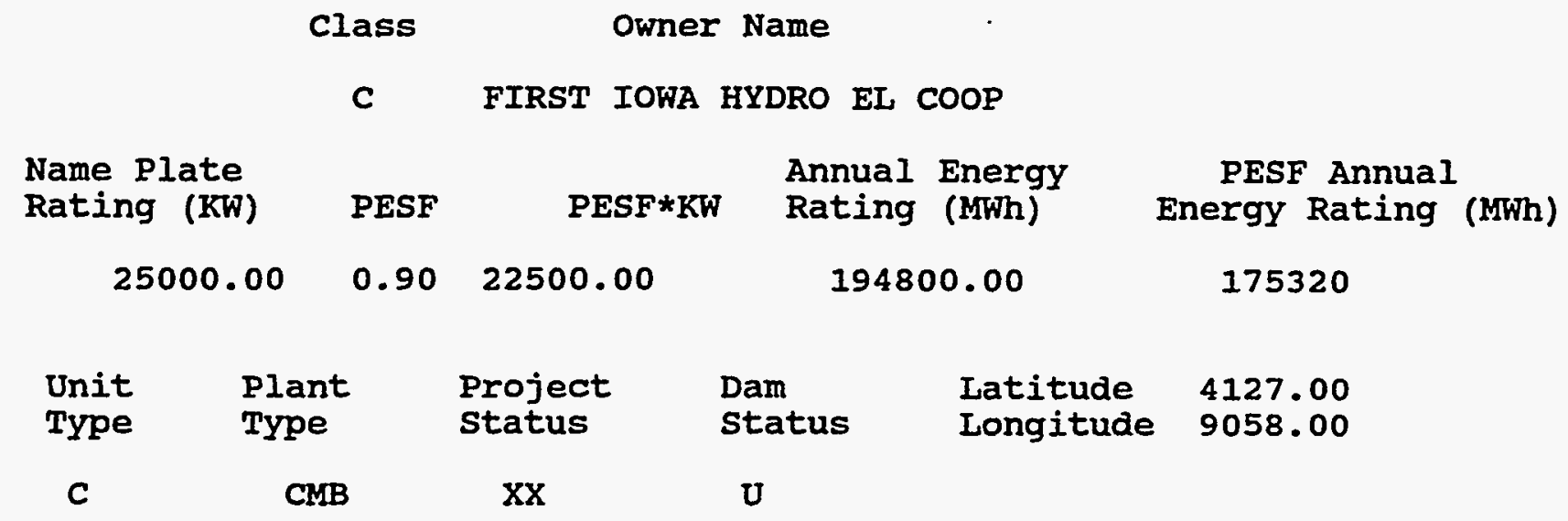

Factor

Exist Prob

Factor

Exist Prob

Wild/scenic Protection

Wild/Scenic Tributary or

0.9

Upstream/Downstream

wila/Scenic Iocation

Cultural Value

Fish Presence Value

Geologic Value

Historic Value

other Value

Recreation value

Scenic Value

Exist
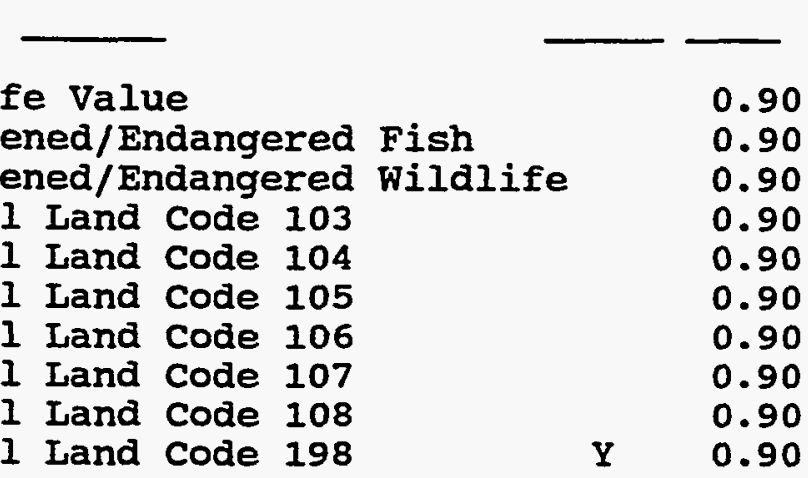
DATE: $11 / 24 / 95$

PAGE NO: 2

FERC

Number

Plant Name

Stream

State

07066

BURIINGTON STREET

IOWA $\mathbf{R}$

Name

IA

county Name

JOHNSON
River Basin

IOWA RIVER BASIN

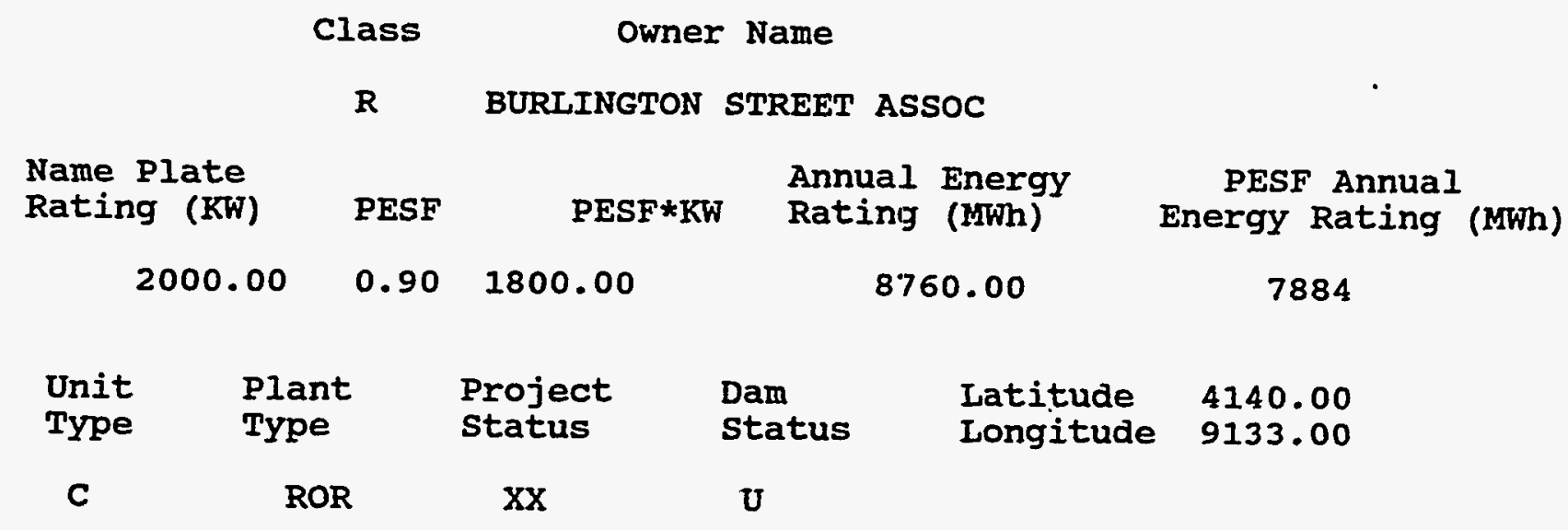

Factor

Wild/Scenic Protection

wild/Scenic Tributary or Upstream/Downstream wild/Scenic Location Cultural Value Fish Presence Value Geologic Value Historic Value other Value Recreation value Scenic Value
Exist Prob

Factor

0.90

wildlife Value Threatened/Endangered Fish Threatened/Endangered Wildlife

0.90 Federal Land Code 103

0.90 Federal Land Code 104

0.90 Federal Land Code 105

0.90 Federal Land Code 106

0.90 Federal Land Code 107

0.90 Federal Land Code 108

0.90 Federal Land Code 198
Exist Prob

\begin{tabular}{ll} 
& \multicolumn{1}{c}{ Exist Prob } \\
Fish & 0.90 \\
Wildife & 0.90 \\
& 0.90 \\
& 0.90 \\
& 0.90 \\
& 0.90 \\
& 0.90 \\
& 0.90 \\
& 0.90 \\
& 0.90
\end{tabular}


FERC

Number

07103
Plant Name

MISSISSIPPI L\&D 17

County Name

LOUISA stream

MISSISSIPPI R

River Basin

IOWA RIVER BASIN
State

Name

IA

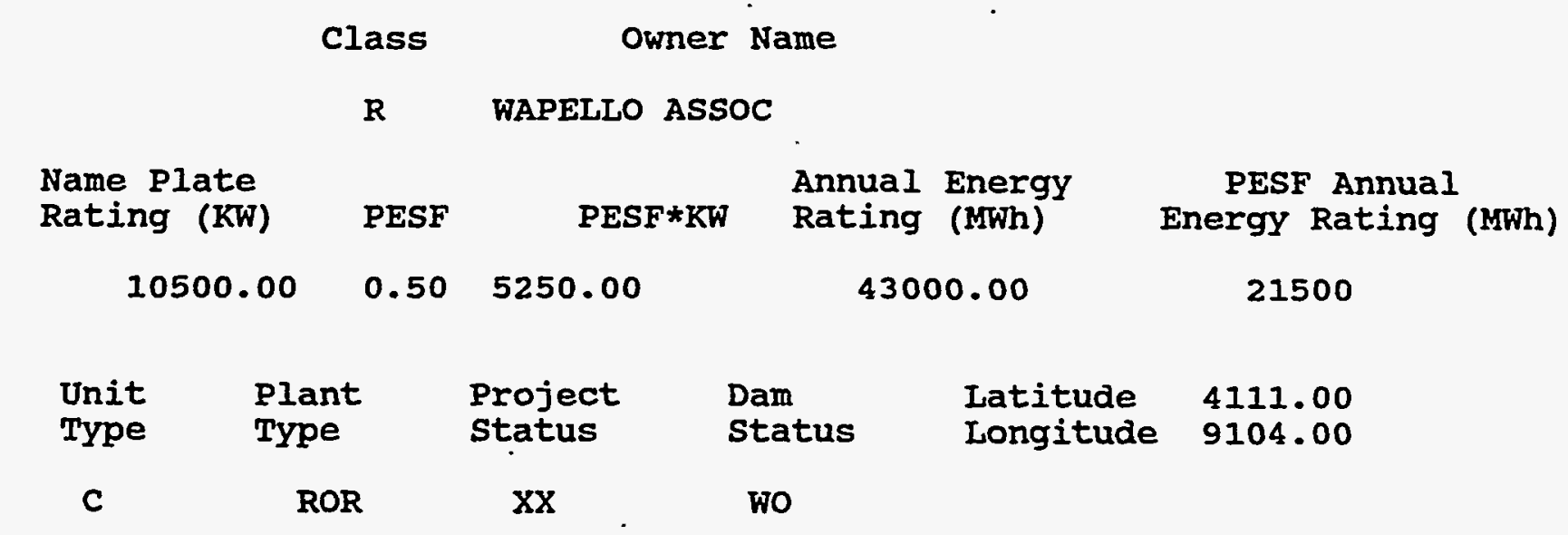

Factor

Exist Prob

Factor

Exist Prob

wild/Scenic Protection

Wild/Scenic Tributary or Upstream/Downstream wild/Scenic Location

Cultural Value

Fish Presence Value

Geologic Value

Historic Value

other Value

Recreation Value

Scenic Value

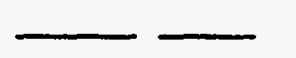

0.90

Wildlife Value

Threatened/Endangered Fish

Threatened/Endangered Wildlife

0.90 Federal Land Code 103

0.90 Federal Land Code 104

0.90 Federal Land Code 105

0.90 Federal Land Code 106

0.90 Federal Land Code 107

0.90 Federal Land Code 108

0.90 Federal Land Code 198 
RESOUR C E D A T A B A S E I I S T I N G

DATE: $11 / 24 / 95$

PAGE NO: 4

FERC

Number

Plant Name

Stream

State

07879 CENTER STREET

DES MOINES $\mathbf{F}$

Name

IA

county Name

POLK

Class

Owner Name

$M$ DES MOINES, CITY OF

Name Plate

Rating (KW)

PESF

PESF*KW

Annual Energy

Rating (MWh)

1860.00

$0.90 \quad 1674.00$

8200.00

PESF Annual

Energy Rating (MWh)

7380

\begin{tabular}{|c|c|c|c|c|}
\hline $\begin{array}{l}\text { Unit } \\
\text { Type }\end{array}$ & $\begin{array}{l}\text { Plant } \\
\text { Type }\end{array}$ & $\begin{array}{l}\text { Project } \\
\text { status }\end{array}$ & $\begin{array}{l}\text { Dam } \\
\text { status }\end{array}$ & $\begin{array}{l}\text { Latitude } \\
\text { Longitude }\end{array}$ \\
\hline
\end{tabular}

c

ROR

XX

พO

Factor

Wild/Scenic Protection

Wild/Scenic Tributary or Upstream/Downstream wild/Scenic Location Cultural Value

Fish Presence Value

Geologic Value

Historic Value

other Value

Recreation Value

Scenic Vaiue
Exist Prob

0.90

Wildlife Value Threatened/Endangered Fish Threatened/Endangered Wildlife

0.90 Federal Land Code 103

0.90 Federal Land Code 104

0.90 Federal Land Code 105

0.90 Federal Iand Code 106

0.90 Federal Land Code 107

0.90 Federal Iand Code 108

0.90 Federal Land Code 198
Exist Prob

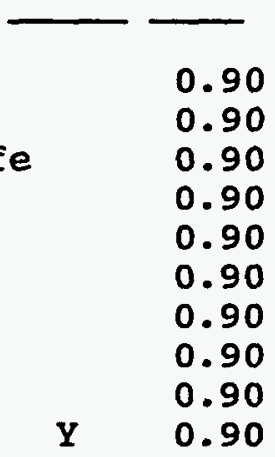


FERC

Number

08096
Plant Name

LINN GROVE MILI

County Name

BUENA VISTA

\section{Stream}

IITTLE SIOUX $R$

River Basin

PLATTE RIVER BASIN
State

Name

IA

$$
\begin{array}{cc}
\text { Class } & \text { Owner Name } \\
\text { R } & \text { IOWA HYDROPOWER DEV CORP }
\end{array}
$$

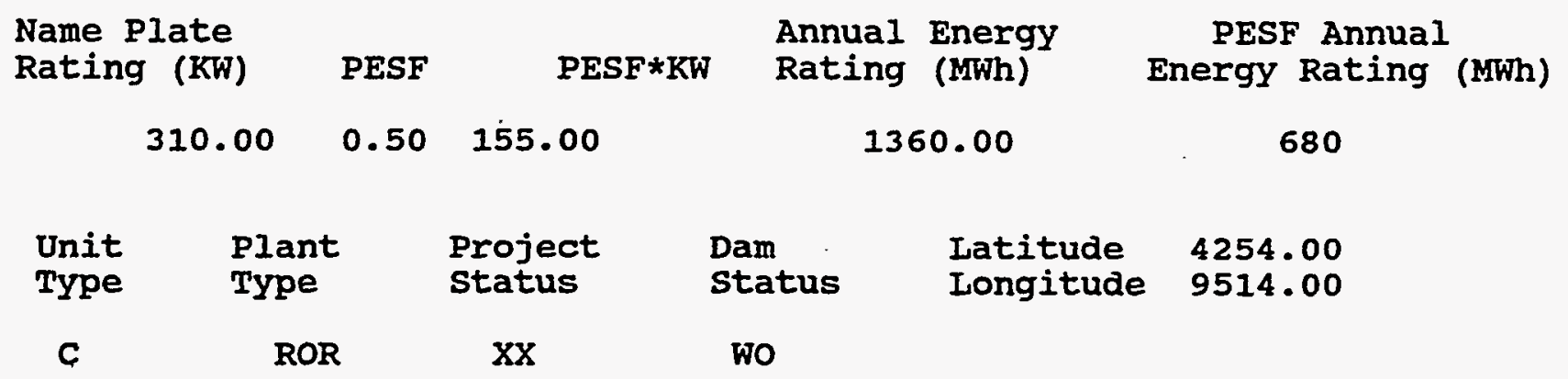

Factor

Wild/Scenic Protection

Wild/Scenic Tributary or Upstream/Downstream wild/Scenic Location Cultural Value

Fish Presence Value

Geologic Value

Historic Value

other Value

Recreation Value

Scenic Value
Exist Prob

Factor

Exist Prob

0.90
0.90
0.90
0.90
0.90
0.90
0.90
0.90
$\quad 0.90$

$\quad 0.90$


FERC

Number

08097
Plant Name

DECORAH UPPER

County Name

WINNESHIEK

Class

$\mathbf{R}$

IOWA

Owner Name

UPPER IOWA :R

stream

River Basin

WISCONSIN RIVER BASIN
State

Name

IA

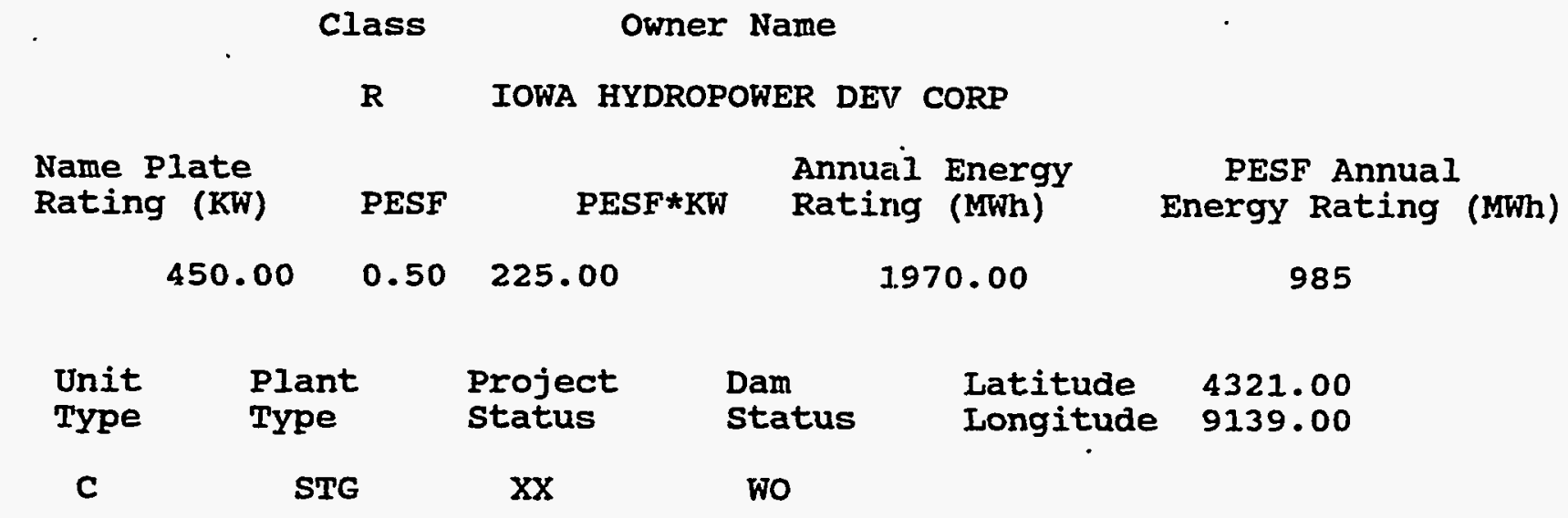

Factor

Wild/Scenic Protection

wild/Scenic Tributary or Upstream/Downstream wild/Scenic Location Cultural value Fish Presence Value Geologic Value Historic Value other Value Recreation Value Scenic Value
Exist Prob

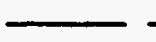

0.90



0.90
0.90
0.75
0.90
0.90
0.90
0.90
0.90

Factor

Exist Prob

wildlife Value Threatened/Endangered Fish Threatened/Endangered Wilalife

$\mathbf{Y}$

0.75

0.90

0.90

0.90

0.90

0.90

0.90

0.90

0.90

0.90 
FERC

Number

Plant Name

08098 LITTLETON DAM

County Name

BUCHANAN state

Name

WAPSIPINICON $R$

IA
River Basin

MISSISSIPPI MAIN STREAM

\section{class Owner Name}

R IOWA HYDROPOWER DEV CORP

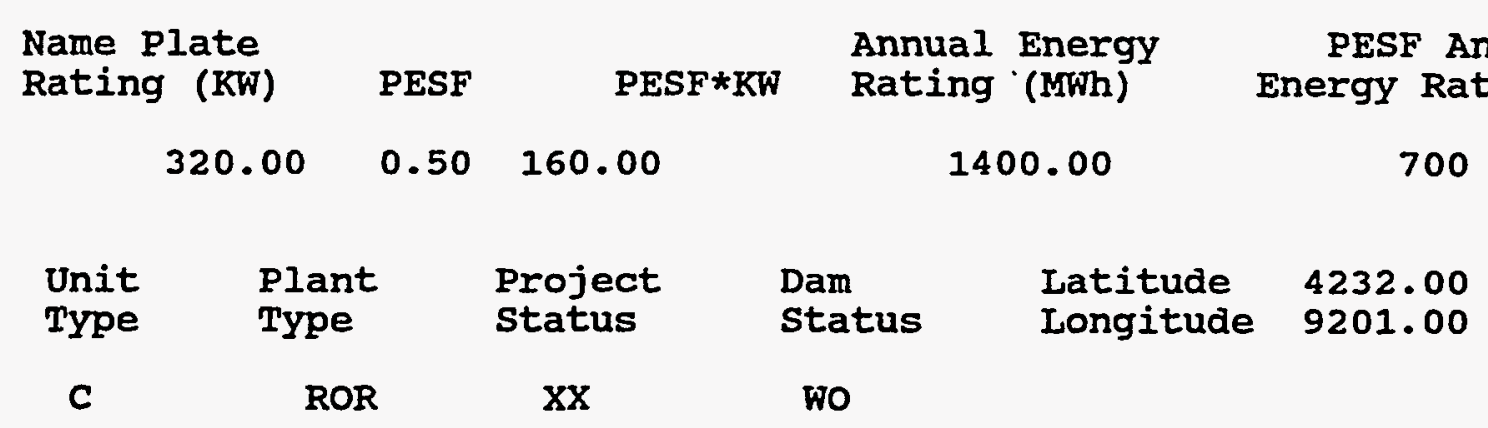

Factor

Exist Prob

Factor

Exist Prob

Wild/Scenic Protection

wild/Scenic Tributary or

0.90 Wildlife Value Threatened/Endangered Fish

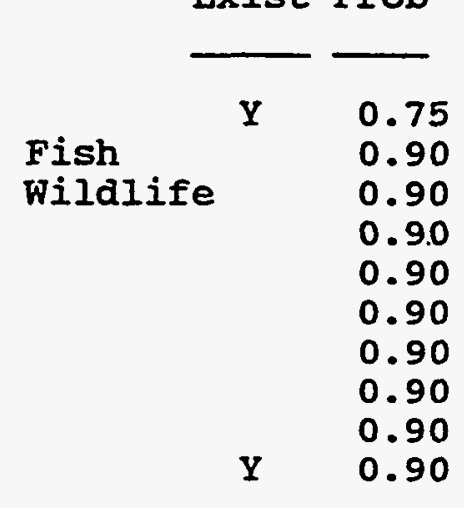

Upstream/Downstream Cultural value Fish Presence Value Geologic Value Historic Value Threatened/Endangered

0.90 Federal Iand Code 103

0.90 Federal Land Code 104

Y 0.75 Federal Land Code 105

Y 0.90 Federal Land Code 106

$\mathrm{Y} \quad 0.75$

Federal Land Code 107

0.90 Federal Land Code 108

Other Value

Scenic Value

0.90
0.90

Federal Land code 198 
R E S O U R E. D A T A B A E I I S T I N G

DATE: $11 / 24 / 95$

PAGE NO: 8

FERC

Number

08105

CENTRAL CITY MILL

county Name

IINN

\section{Class}

Owner Name

$\mathrm{R}$ IOWA HYDROPOWER DEV CORP

Name Plate

Rating (KW)

PESF

PESF*KW

Annual Energy

640.00

$0.50 \quad 320.00$

1360.00

Dam

Status

Latitude

Iongitude

4212.00

status

XX

WO

$\begin{array}{cccccc}\text { Unit } & \text { Plant } & \begin{array}{l}\text { Project } \\ \text { Status }\end{array} & \begin{array}{l}\text { Dam } \\ \text { Status }\end{array} & \begin{array}{l}\text { Latitude } \\ \text { Longitude }\end{array} & 4212.00 \\ 9131.00\end{array}$

state

Name

IA
Factor

Wild/Scenic Protection Wild/Scenic Tributary or Upstream/Downstream Wild/Scenic Location Cultural Value Fish Presence Value Geologic Value Historic Value other Value Recreation value Scenic Value

\section{Exist Prob}

Factor

Exist Prob

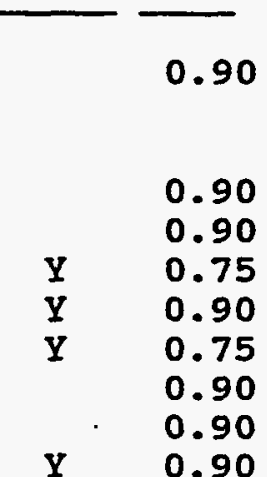

Wildlife Value Threatened/Endangered Fish Threatened/Endangered Wildlife

$\mathbf{Y}$ Federal Land code 103 Federal Land Code 104 Federal Land Code 105 Federal Land Code 106 Federal Land Code 107 Federal Land Code 108 Federal Land Code 198
PESF Annual Energy Rating (MWh)

680 
DATE: $11 / 24 / 95$

FERC

Number

08109
Plant Name

STEAMBOAT ROCK

County Name

HARDIN
PAGE NO: 9

State

Name

IOWA $\mathrm{R}$

IA
River Basin

IOWA RIVER BASIN

\section{Class Owner Name \\ $R$ IOWA HYDROPOWER DEV CORP}

Name Plate

Rating (KW)

PESF

PESF*KW

Annual Energy

280.00

$0.50 \quad 140.00$

1230.00

PESF Annual

Energy Rating (MWh)

615

\begin{tabular}{|c|c|c|c|c|c|}
\hline $\begin{array}{l}\text { Unit } \\
\text { Type }\end{array}$ & $\begin{array}{l}\text { Plant } \\
\text { Type }\end{array}$ & $\begin{array}{l}\text { Project } \\
\text { Status }\end{array}$ & $\begin{array}{l}\text { Dam } \\
\text { status }\end{array}$ & $\begin{array}{l}\text { Latitude } \\
\text { Longitude }\end{array}$ & $\begin{array}{l}4224.00 \\
9304.00\end{array}$ \\
\hline C & ROR & $x X$ & พ० & & \\
\hline
\end{tabular}

Factor

Exist Prob

Factor

Exist Prob

Wild/Scenic Protection

Wild/Scenic Tributary or

Upstream/Downstream

wild/Scenic Location

Cultural Value

Fish Presence Value

Geologic Value

Historic Value

other Value

Recreation value

Scenic Value

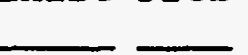

\subsection{0}

Wildlife Value

Threatened/Endangered Fish

Threatened/Endangered wildlife

0.90 Federal Land Code 103

0.90 Federal Land Code 104

Y $\quad 0.75$ Federal Land code 105

0.90 Federal Land Code 106

0.90 Federal Land Code 107

0.90 Federal Land Code 108

Y $\quad 0.75$ Federal Land Code 198

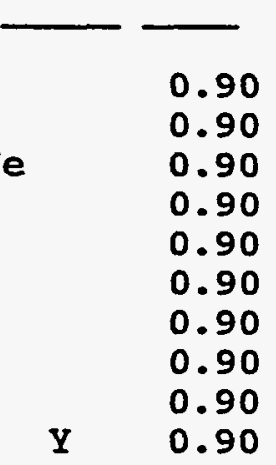


DATE: $11 / 24 / 95$

PAGE NO: 10

FERC

Number

08110

AIDEN MILI

County Name

HARDIN
Plant Name

Stream
State

Name

IOWA $\mathbf{R}$

IA
River Basin

IOWA RIVER EASIN

$\begin{array}{cc}\text { Class } & \text { Owner Name } \\ R & \text { IOWA HYDROPOWER DEV CORP }\end{array}$

\begin{tabular}{|c|c|c|c|c|c|c|c|c|}
\hline $\begin{array}{l}\text { Name } \\
\text { Rating }\end{array}$ & & PESF & PE: & & $\begin{array}{l}\text { Annu } \\
\text { Rati }\end{array}$ & $\begin{array}{l}\text { Energy } \\
\text { (MWh) }\end{array}$ & & $\begin{array}{l}\text { PESF Ar } \\
\text { ergy Rat }\end{array}$ \\
\hline & .00 & 0.50 & 110.00 & & & 70.00 & & 485 \\
\hline $\begin{array}{l}\text { Unit } \\
\text { Type }\end{array}$ & $\begin{array}{l}\text { PIa } \\
\text { TyI }\end{array}$ & & $\begin{array}{l}\text { Project } \\
\text { Status }\end{array}$ & & Eatus & $\begin{array}{l}\text { Latit } \\
\text { Longi }\end{array}$ & & $\begin{array}{l}4230.00 \\
9322.00\end{array}$ \\
\hline C & & & $\mathrm{XX}$ & & vo & & & \\
\hline
\end{tabular}

Factor

Exist Prob

wild/Scenic Protection

Wild/Scenic Tributary or

Upstream/Downstream

wild/Scenic Location

Cultural Value

Fish Presence Value

Geologic Value

Historic Value

other Value

Recreation Value

Scenic Value

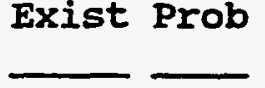

\subsection{0}

Wildlife Value

Threatened/Endangered wildlife

0.90 Federal Land Code 103

0.90 Federal Land Code 104

Y $\quad 0.75$ Federal Land Code 105

0.90 Federal Land Code 106

0.90 Federal Land Code 107

0.90 Federal Iand Code 108

$Y \quad 0.75$ Federal Land Code 198
Exist Prob

0.90

0.90

0.90

0.90

0.90

0.90

0.90

0.90

0.90

Y $\quad 0.90$ 
DATE: $11 / 24 / 95$

FERC

Number

Plant Name

08111 WATERLOO

County Name

BLACK HAWK
PAGE NO: 11

State

Name

IA
CEDAR R

River Basin

IOWA RIVER BASIN

\section{Class Owner Name}

R IOWA HYDROPOWER DEV CORP

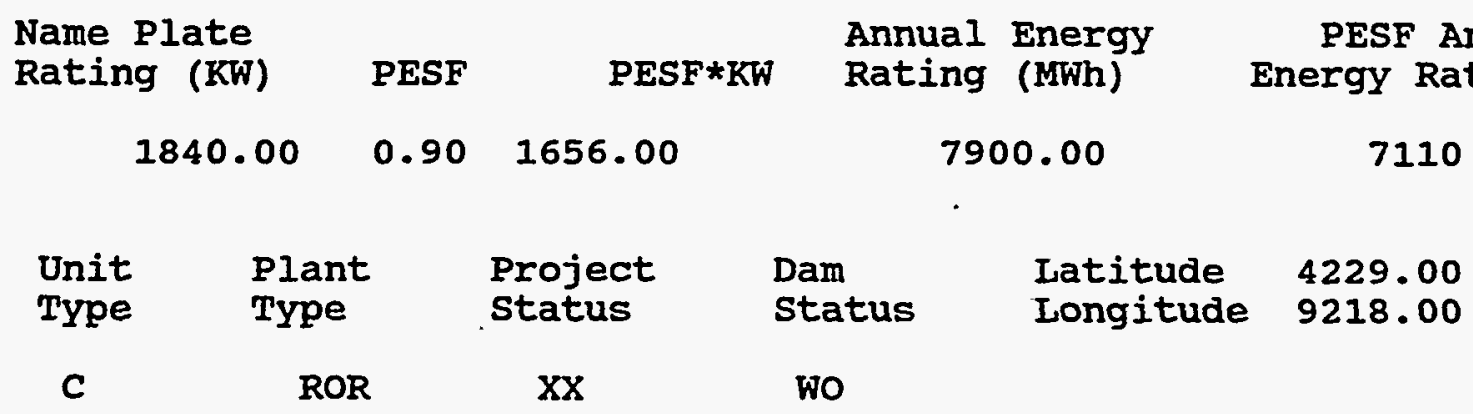

Factor

Exist Prob

Factor

Exist Prob

Wild/Scenic Protection

Wild/Scenic Tributary or Upstream/Downstream Wild/Scenic Location Cultural Value Fish Presence Value Geologic Value Historic Value other Value Recreation Value Scenic Value
0.90 Wildlife Value Threatened/Endangered Fish Threatened/Endangered Wildlife

\section{Exist Prob}

$\begin{array}{ll}0.90 & \text { Federal Land Code } 103 \\ 0.90 & \text { Federal Land Code } 104\end{array}$

0.90 Federal Land Code 105

0.90 Federal Land Code 106

0.90 Federal Land Code 107

0.90 Federal Land Code 108

0.90 Federal Land Code 198

0.90
0.90

0.90

0.90

0.90

0.90

0.90

0.90

0.90

0.90

0.90 
FERC Number

08184 QUAKER MILLS

County Name DELAWARE
Plant Name

Stream

MAQUOKETA: $R$

River Basin

MAQUOKETA RIVER BASIN
State

Name

IA

$$
\begin{array}{cc}
\text { Class } & \text { Owner Name } \\
\mathbf{R} & \text { IOWA HYDROPOWER DEV CORP }
\end{array}
$$

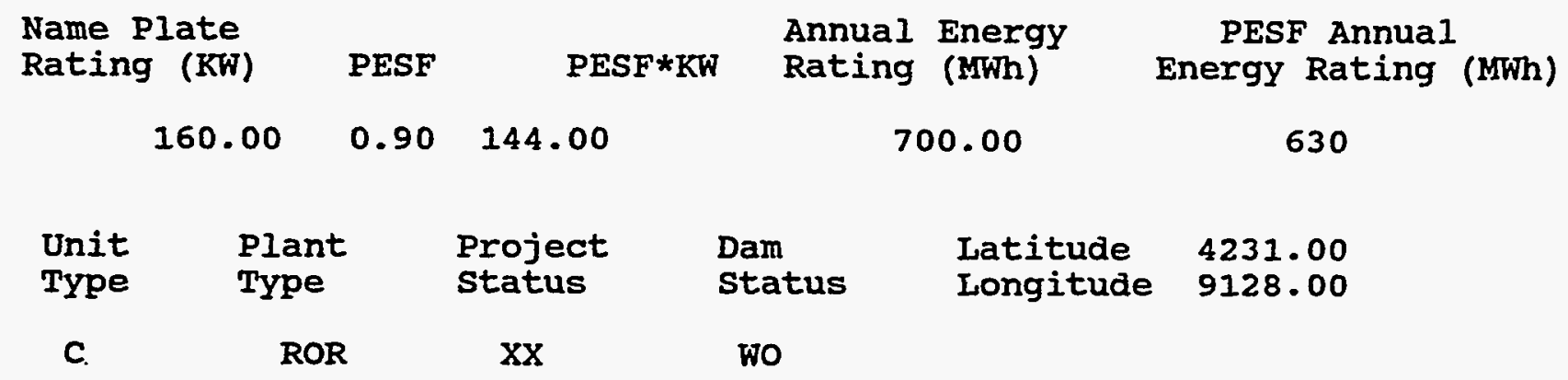

Factor

Wild/Scenic protection Wild/Scenic Tributary or Upstream/Downstream wild/Scenic Iocation Cultural value Fish Presence Value Geologic Value Historic Value Other Value Recreation Value Scenic Value
Exist Prob

Factor

Exist Prob

0.90

Wildlife Value Threatened/Endangered Fish Threatened/Endangered Wildlife

0.90 Federal Land Code 103

0.90 Federal I and Code 104

0.90 Federal Land Code 105

0.90 Federal Land Code 106

0.90 Federal Land Code 107

0.90 Federal Land Code 108

0.90 Federal Land Code 198
0.90

0.90

0.90

0.90

0.90

0.90

0.90

0.90

0.90

0.90 
DATE: $11 / 24 / 95$

FERC

Number

08642

ELKADER

County Name

CLAYTON

\section{Class Owner Name \\ R ENERGY RESOURCES GROUP}

\begin{abstract}
Name Plate
\end{abstract}
Rating (KW)

PESF

Annual Energy

500.00

$0.50 \quad 250.00$

PESF*KW

Rating (MWh)

PESF Annual

Energy Rating (MWh)

$$
2497.00
$$

1248.50
State

Name

IA
River Basin

MAQUOKETA RIVER BASIN

$\begin{array}{cccccc}\text { Unit } & \text { Plant } & \text { Project } & \text { Dam } & \text { Latitude } & 4251.00 \\ \text { Type } & \text { Type } & \text { Status } & \text { Status } & \text { Longitude } & 9124.00 \\ \text { C } & \text { ROR } & \text { XX } & \text { WO } & & \end{array}$

Factor

Wild/Scenic Protection

Wild/Scenic Tributary or Upstream/Downstream wild/Scenic Location Cultural Value Fish Presence Value Geologic Value Historic Value other Value Recreation Value Scenic Value
Exist Prob

Factor

Exist Prob

\subsection{0}

Wildlife Value Threatened/Endangered Fish Threatened/Endangered wildlife

0.90 Federal Land Code 103

Y $\quad 0.75$ Federal Land Code 104

$Y \quad 0.75$ Federal Land Code 105

Y $\quad 0.90$ Federal Land Code 106

Y 0.75 Federal Land Code 107

Y 0.75 Federal Land Code 108

Y 0.75 Federal.Land Code 198 
DATE: $11 / 24 / 95$

PAGE NO: 14

FERC

Number

Plant Name

Stream

state

Name

08691 CORALVILIE MILL

IOWA $\mathbf{R}$

County Name

River Basin

JOHNSON

IOWA RIVER BASIN

$$
\begin{array}{cr}
\text { Class } & \text { Owner Name } \\
\text { M } & \text { IOWA CITY, CITY OF }
\end{array}
$$

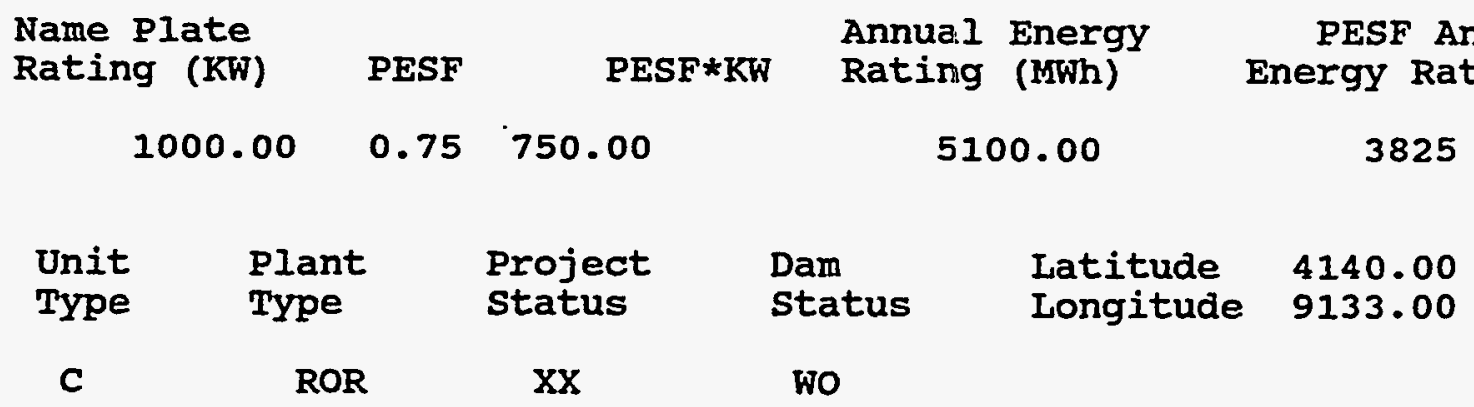

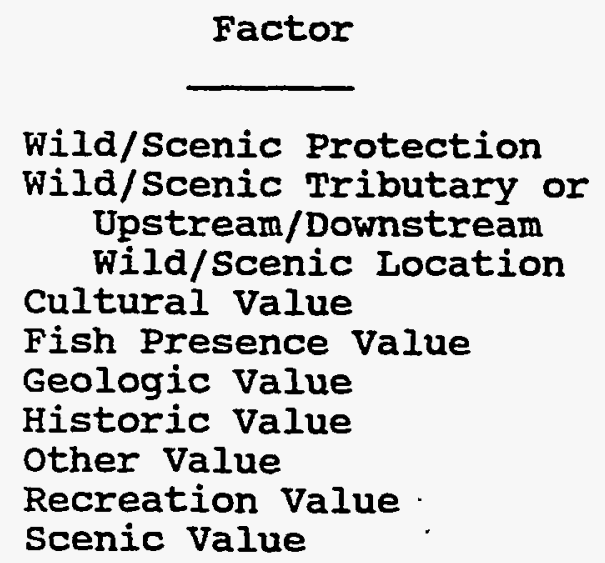

Exist Prob

Factor

Exist Prob

\begin{tabular}{cc}
\hline & \\
& \\
& \\
& \\
& \\
& 0.90 \\
& 0.90 \\
& 0.90 \\
& 0.90 \\
& 0.90 \\
& 0.90 \\
& 0.90 \\
& 0.75 \\
& 0.90
\end{tabular}

wilalife Value Threatened/Endangered Fish Threatened/Endangered Wildlife IA 
DATE: $11 / 24 / 95$

PAGE NO: 15

FERC

Number

08741

REDFIELD

county Name

DALLAS
Plant Name

Stream state

Name

IA

$$
\text { River Basin }
$$

DES MOINES RIVER BASIN

\section{Class Owner Name}

$R$ DALLAS COUNTY HYDRO CO

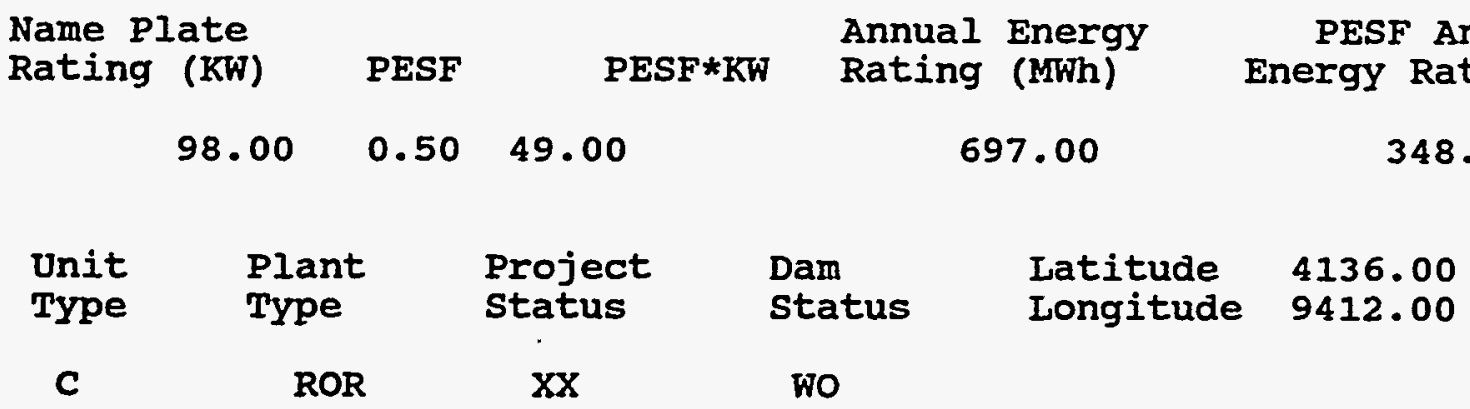

Factor

Exist Prob

Factor

Exist Prob

Wild/Scenic Protection

wild/Scenic Tributary or Upstream/Downstream wild/Scenic Location Cultural Value Fish Presence Value Geologic Value Historic Value other Value Recreation Value Scenic Value

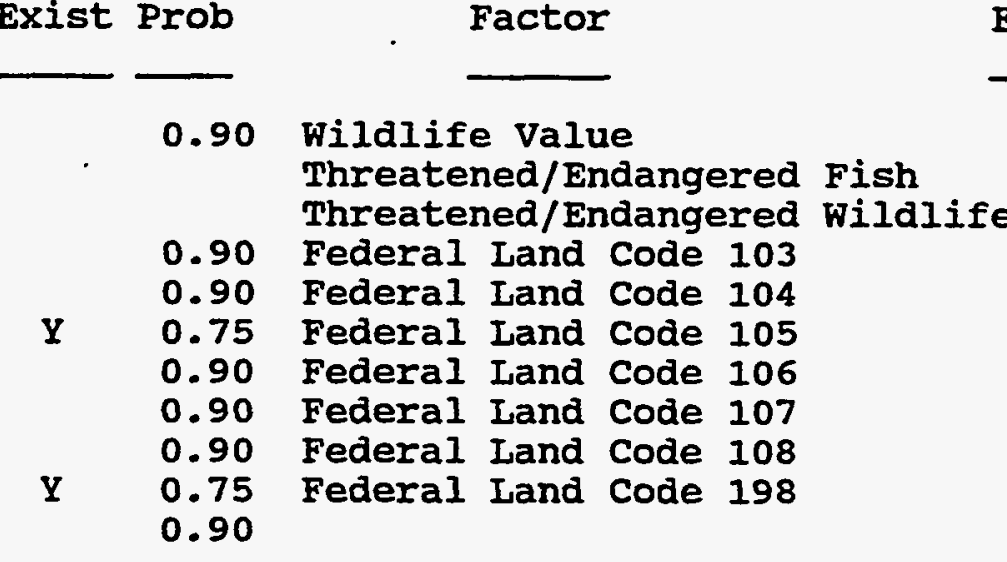


FERC

Number

08955

HENRY
OAKI.AND MIILS

county Name

Plant Name
River Basin

MISSISSIPPI MAIN STREAM

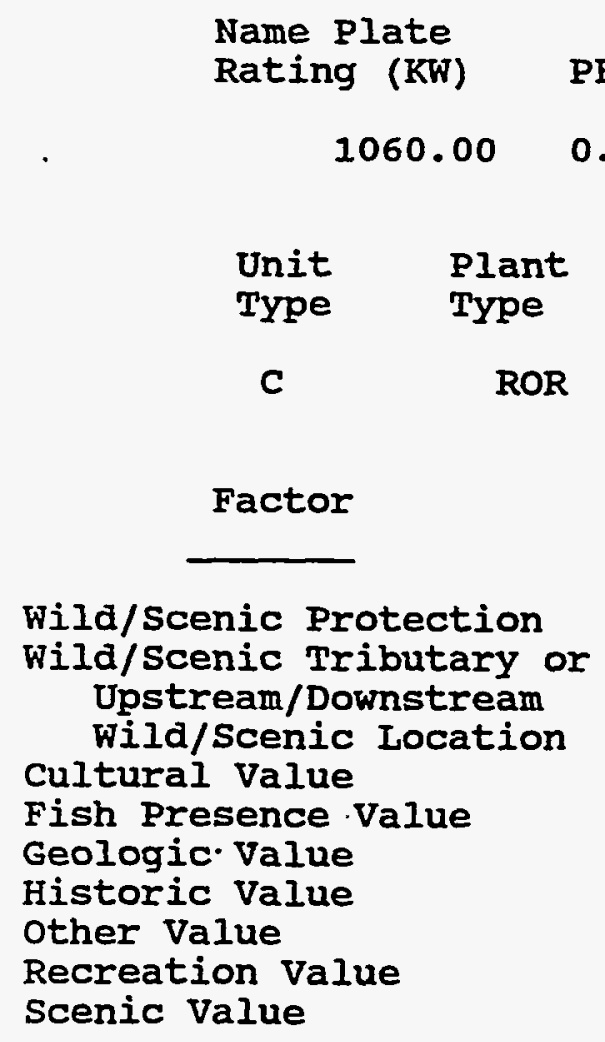

Wild/Scenic Protection wild/Scenic Tributary or Upstream/Downstream wild/Scenic Iocation

\section{Factor Cultural value Fish Presence value Geologic. Value Historic Value other Value Recreation Value \\ Scenic Value}

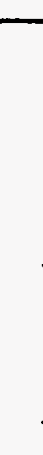

XX

Factor
Exist Prob

\section{Annual Energy}

4813.00
Iatitude Longitude

$\begin{array}{lll}\text { Dam } & \text { Latitude } & 4056.00 \\ \text { Status } & \text { Longitude } & 9137.00\end{array}$

พอ
PESF Annual Energy Rating (MWh)

$$
2406.50
$$

Exist Prob

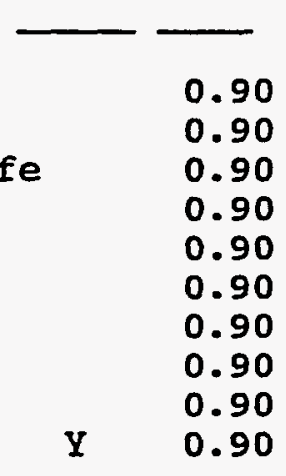


DATE: $11 / 24 / 95$

PAGE NO: 17

FERC

Number

09278

CEDAR FALLS

County Name

BLACK HAWK
State

Name

IA
CEDAR $R$

River Basin

IOWA RIVER BASIN

\section{Class Owner Name \\ R CEDAR FALLS DEV ITD}

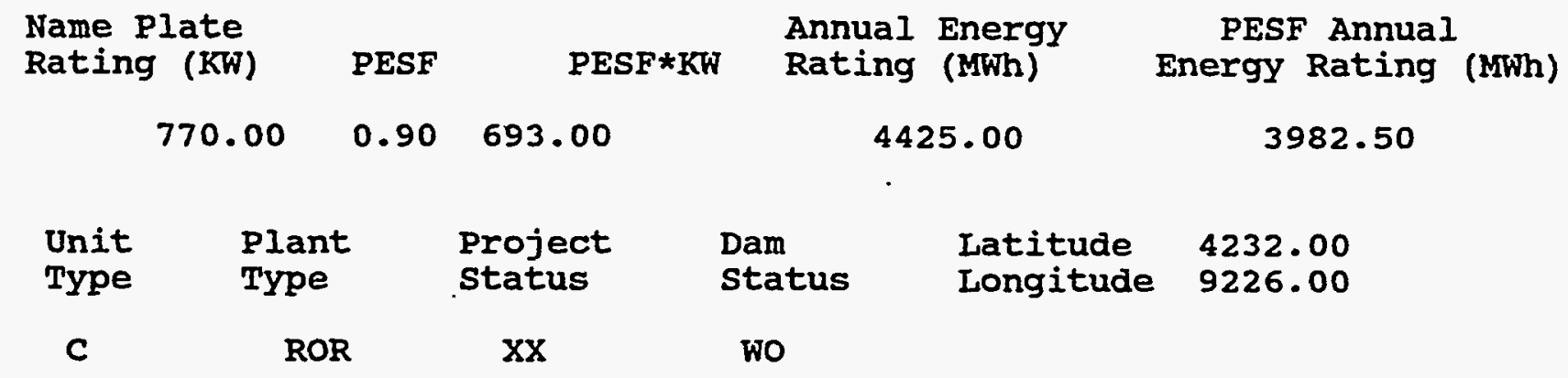

Factor

Exist Prob

0.90

Wildlife Value Threatened/Endangered Fish Threatened/Endangered Wildlife

0.90 Federal Land Code 103

0.90 Federal Land Code 104

0.90 Federal Land Code 105

0.90 Federal Iand Code 106

0.90 Federal Land Code 107

0.90 Federal Land Code 108

0.90 Federal Land Code 198
Exist Prob

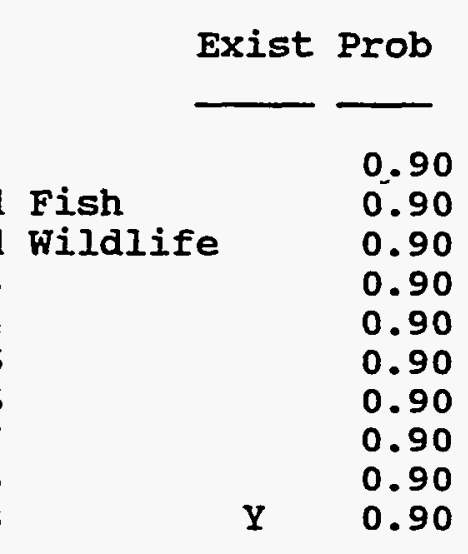

0.90 
DATE: $11 / 24 / 95$

PAGE NO: 18

FERC

Number

Plant Name

Stream

state

Name

09327 DECORAH LOWER

UPPER IOWA $R$

IA

county Name

WINNESHIEK
River Basin

WISCONSIN RIVER BASIN

$$
\begin{array}{cc}
\text { Class } & \text { Owner Name } \\
\text { R } & \text { DECORAH HYDRO ASSOC }
\end{array}
$$

Name Plate

Rating ( $\mathrm{KW}$ )

PESF

Annual Energy

PESF*KW

Rating (MWh)

PESF Annual

670.00

$0.75 \quad 502.50$

2900.00

\begin{tabular}{|c|c|c|c|c|c|}
\hline $\begin{array}{l}\text { Unit } \\
\text { Type }\end{array}$ & $\begin{array}{l}\text { Plant } \\
\text { Type }\end{array}$ & $\begin{array}{l}\text { Project } \\
\text { Status }\end{array}$ & $\begin{array}{l}\text { Dam } \\
\text { status }\end{array}$ & $\begin{array}{l}\text { Latitude } \\
\text { Longitude }\end{array}$ & $\begin{array}{l}4321.00 \\
9139.00\end{array}$ \\
\hline C & STG & $x x$ & พั & & \\
\hline
\end{tabular}

2175

Factor

Exist Prob

Factor

Exist Prob

Wild/Scenic Protection

wild/Scenic Tributary or Upstream/Downstream wild/Scenic Location Cultural Value

Fish Presence Value Geologic Value Historic Value Other Value Recreation. Value Scenic Vailue

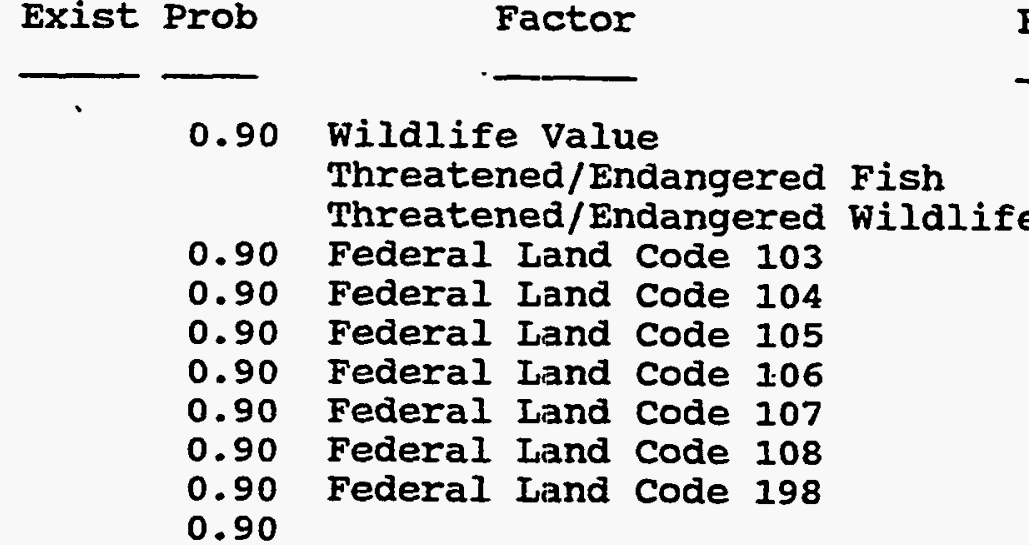

0.75

0.90

0.90

0.90

0.90

0.90

0.90

0.90

0.90

Y 
RES O UR C E D A T A B A S E I I S T I N G

DATE: $11 / 24 / 95$

PAGE NO: 19

FERC

Number

Plant Name

stream

state

09882 ADEL MILI

RACCOON $\mathbf{R}$

Name

County Name

River Basin

DALIAAS

DES MOINES RIVER BASIN

IA

PESF Annual

Energy Rating (MWh)

\begin{tabular}{|c|c|c|c|c|}
\hline $\begin{array}{l}\text { Name Plate } \\
\text { Rating (KW) }\end{array}$ & PESF & $\mathrm{PESF} * \mathrm{KW}$ & $\begin{array}{l}\text { Annual Energy } \\
\text { Rating (MWh) }\end{array}$ & $\begin{array}{l}\text { PESF Annual } \\
\text { Energy Rating (MWh) }\end{array}$ \\
\hline 420.00 & 0.90 & $378^{\circ} .00$ & 2222.00 & 1999.80 \\
\hline
\end{tabular}

\begin{tabular}{|c|c|c|c|c|c|}
\hline $\begin{array}{l}\text { Unit } \\
\text { Type }\end{array}$ & $\begin{array}{l}\text { Plant } \\
\text { Type }\end{array}$ & $\begin{array}{l}\text { Project } \\
\text { Status }\end{array}$ & $\begin{array}{l}\text { Dam } \\
\text { Status }\end{array}$ & $\begin{array}{l}\text { Latitude } \\
\text { Longitude }\end{array}$ & $\begin{array}{l}4138.00 \\
9401.00\end{array}$ \\
\hline C & ROR & $X X$ & พั & & \\
\hline
\end{tabular}

Factor

Exist Prob

Factor

Exist Prob

Wild/Scenic Protection

Wild/Scenic Tributary or

0.90

Wildlife value

Threatened/Endangered Fish

Threatened/Endangered wildlife

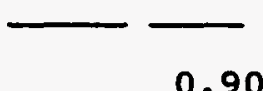

Upstream/Downstream wild/Scenic Location

0.90 Federal Land Code 103

0.90 Federal Land Code 104

0.90

0.90

0.90

0.90

Federal Land Code 105

0.90

0.90

Federal Land code 107

0.90

Fish Presence Value

0.90

Federal Land Code 108

0.90

Historic Value

0.90

Federal Land Code 198

0.90

0.90

0.90

0.90

Recreation Value

Scenic Value 
DATE: $11 / 24 / 95$

PAGE NO: 20

FERC

Number

09891

CHARLES CITY MILL

county Name

FLOYD
Plant Name

county Name

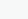

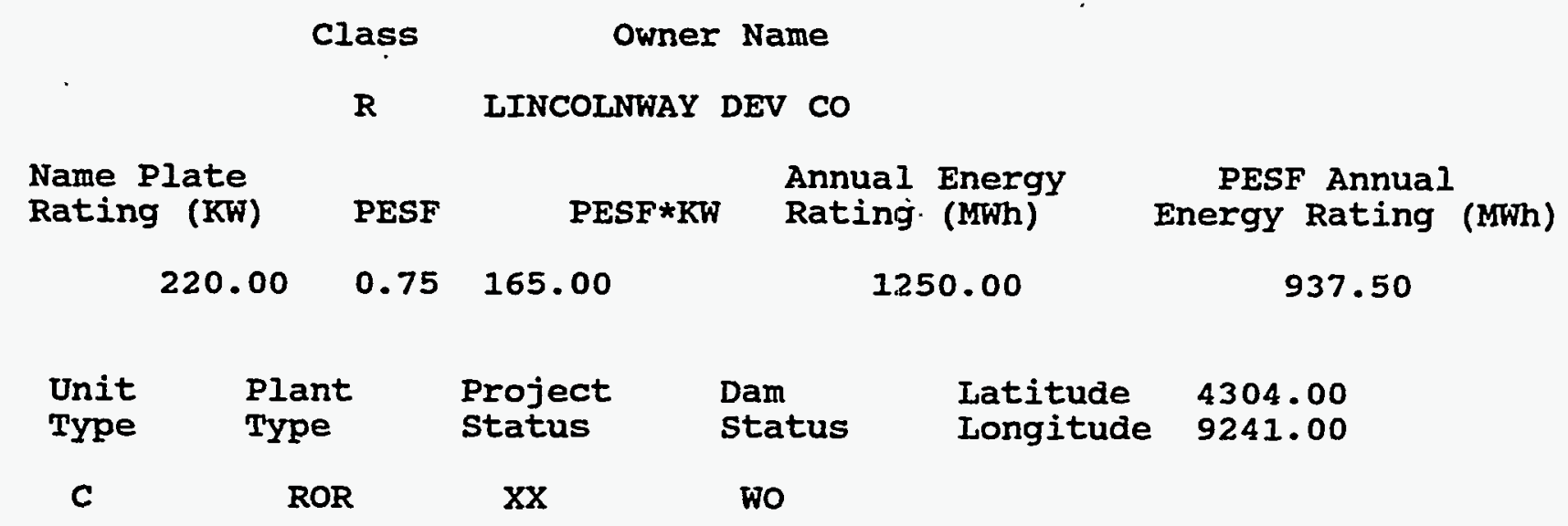

Factor

wild/Scenic Protection wild/Scenic Tributary or Upstream/Downstream wild/Scenic Location Cultural Value Fish Presence Value Geologic Value Historic Value other value Recreation Value Scenic Value
Exist Prob

0.90

Threatened/Endangered Fish

0.90 Federal Iand Code 103

0.90 Federal Iand Code 104

0.90 Federal Land Code 105

0.90 Federal Land Code 106

0.90 Federal Land Code 107

0.90 Federal Land Code 108

0.90 Federal. Land Code 198
State

Name

IA

IOWA RIVER BASIN

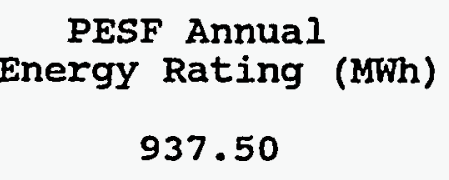

Exist Prob

0.90

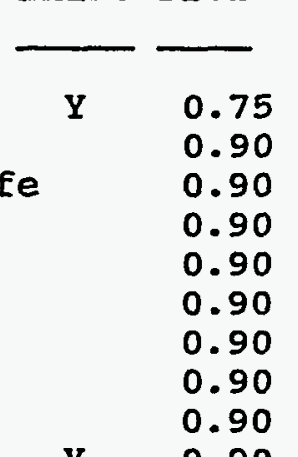


DATE: $11 / 24 / 95$

PAGE NO: 21

FERC

Number

09892
Plant Name

MANCHESTER MILI

County Name

DELAWARE
Stream

MAQUOKETA $\mathbf{R}$

River Basin

MAQUOKETA RIVER BASIN state

Name

IA

\section{Class Owner Name \\ $R$ IINCOLNWAY DEV CO}

\begin{tabular}{|c|c|c|c|c|c|c|}
\hline \multicolumn{2}{|c|}{$\begin{array}{l}\text { Name Plate } \\
\text { Rating (KW) }\end{array}$} & PESF & PESF*KW & \multicolumn{2}{|c|}{$\begin{array}{l}\text { Annual Energy } \\
\text { Rating (MWh) }\end{array}$} & $\begin{array}{l}\text { PESF Annual } \\
\text { Energy Rating (MWh) }\end{array}$ \\
\hline & 150.00 & 0.75 & 112.50 & & 750.00 & 562.50 \\
\hline $\begin{array}{l}\text { Unit } \\
\text { Type }\end{array}$ & $\begin{array}{l}\text { Pla } \\
\text { TyP }\end{array}$ & & $\begin{array}{l}\text { Project } \\
\text { Status }\end{array}$ & $\begin{array}{l}\text { Dam } \\
\text { status }\end{array}$ & $\begin{array}{l}\text { Latitude } \\
\text { Longitude }\end{array}$ & $\begin{array}{l}4229.00 \\
9128.00\end{array}$ \\
\hline C & & & $\mathrm{XX}$ & พо & & \\
\hline
\end{tabular}

Factor

Exist Prob

Factor

Exist Prob

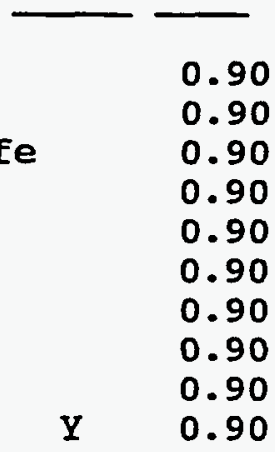

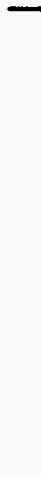

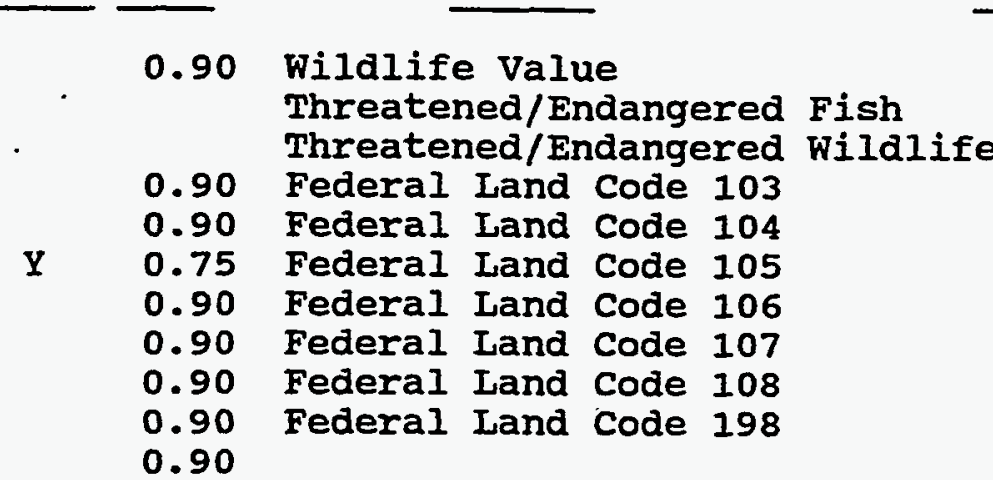

Wild/Scenic Protection Wild/Scenic Tributary or Upstream/Downstream wild/Scenic Location Cultural Value Fish Presence Value Geologic·Value Historic Value other Value Recreation Value Scenic Value 
DATE: $11 / 24 / 95$

PAGE NO: 22

FERC

Number

09893 HOPKINTON DAM

County Name

DELAWARE
Plant Name

Class

R IINCOLNWAY DEV CO

Name Plate

Rating (KW)

$$
250.00
$$

Annual Energy Rating (MWh)

$$
1100.00
$$

\section{Latitude} Longitude
State

Name

IA

$\begin{array}{ccccc}\text { Unit } & \text { Plant } & \begin{array}{l}\text { Project } \\ \text { Status }\end{array} & \begin{array}{l}\text { Dam } \\ \text { Status }\end{array} & \begin{array}{l}\text { Latitude } \\ \text { Longitude }\end{array} \\ \text { Type } & \text { Type } & \text { S116.00 } \\ \text { C } & \text { ROR } & \text { XX } & \text { wo } & \end{array}$

Factor

Exist Prob

Wild/Scenic Protection

wild/Scenic Tributary or Upstream/Downstream wild/Scenic Location

Cultural Value

Fish Presence Value

Geologic Value

Historic Value

other Value

Recreation Value

Scenic Value

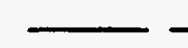

\subsection{0}

0.90

0.90

0.90

0.90

0.90

0.90

0.90

0.90
Factor
Exist Prob

PESF Annual Energy Rating (MWh)

990

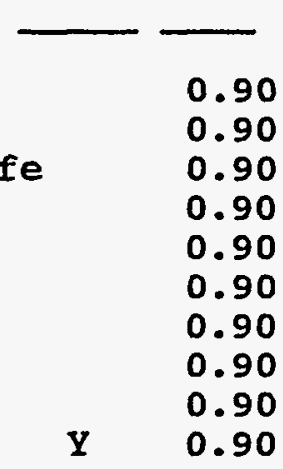


DATE: $11 / 24 / 95$

PAGE NO: 23

FERC

Number

10229
Plant Name

INDEPENDENCE MILL

County Name

BUCHANAN stream

WAPSIPINICON $R$

River Basin

MISSISSIPPI MAIN STREAM state

Name

IA

class Owner Name

$R$ INDEPENDENCE HYDRO CORP

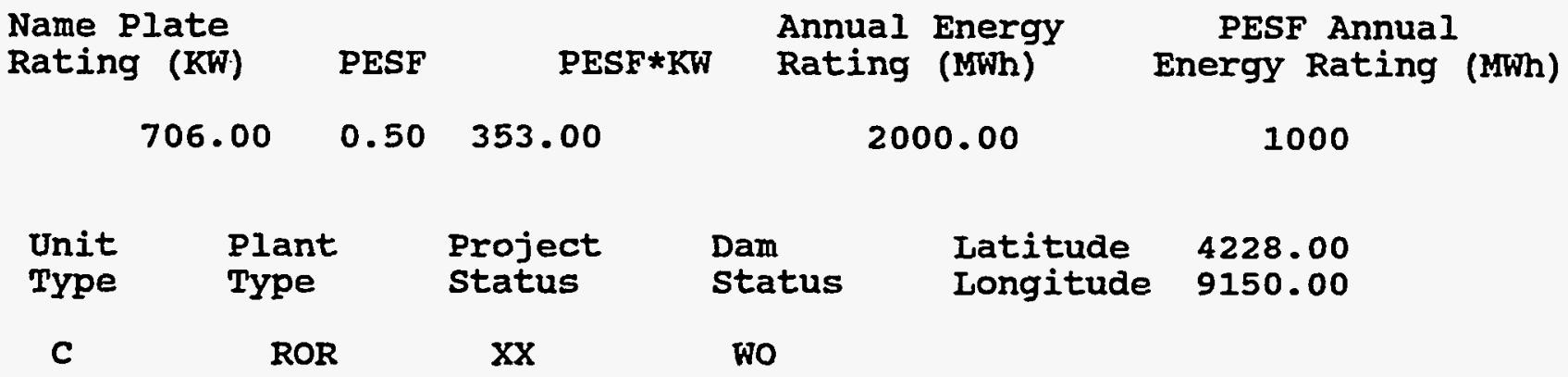

Factor

Wild/Scenic Protection

Wild/Scenic Tributary or Upstream/Downstream Wild/Scenic Location Cultural value Fish Presence Value Geologic Value Historic Value other Value Recreation Value Scenic Value
Exist Prob

Factor

Exist Prob

\subsection{0}

Wildlife Value

Threatened/Endangered Fish

Threatened/Endangered Wildlife

0.90 Federal Land Code 103

0.90 Federal Land Code 104

Y 0.75 Federal Iand Code 105

Y 0.90 Federal Land Code 106

Y 0.75 Federal Land code 107

0.90 Federal Land Code 108

0.90 Federal Land Code 198

$\mathrm{Y} \quad 0.90$

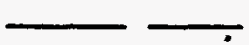

$\mathrm{Y}$

0.75

0.90

0.90

0.90

0.90

0.90

0.90

0.90

0.90

Y $\quad 0.90$ 
DATE: $11 / 24 / 95$

PAGE NO: 24

FERC

Number

Plant Name

Stream

state

10747 RATHBUN DAM

CHARITON $\mathbf{R}$

Name

County Name

River Basin

APPANOOSE

MISSOURI MAIN STREAM

\author{
Class Owner Name \\ $R$ IOWA HYDRO DEV CORP
}

\begin{tabular}{|c|c|c|c|c|c|c|c|}
\hline \multicolumn{2}{|c|}{ Name Plate } & PESF & PESF*KW & \multicolumn{2}{|c|}{$\begin{array}{l}\text { Annual Energy } \\
\text { Rating (MWh) }\end{array}$} & $\begin{array}{l}\text { PESF Annual } \\
\text { Energy Rating }\end{array}$ & (MWh) \\
\hline \multicolumn{2}{|c|}{1910.00} & 0.75 & 1432.50 & \multicolumn{2}{|c|}{5150.00} & 3862.50 & \\
\hline $\begin{array}{l}\text { Unit } \\
\text { Type }\end{array}$ & $\begin{array}{l}\text { PI } \\
\text { TYI }\end{array}$ & & $\begin{array}{l}\text { Project } \\
\text { status }\end{array}$ & $\begin{array}{l}\text { Dam } \\
\text { Status }\end{array}$ & $\begin{array}{l}\text { Latitude } \\
\text { Iongitude }\end{array}$ & $\begin{array}{l}4050.00 \\
9253.00\end{array}$ & \\
\hline c & & & PO & พо & & & \\
\hline
\end{tabular}

Factor

Exist Prob

Factor

Exist Prob

Wild/Scenic Protection

wild/Scenic Tributary or

Upstream/Downstream

wild/Scenic Location

Cultural Value

Fish Presence Value

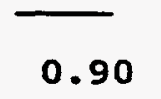

0.90 Wildlife Value

Threatened/Endangered Fish

Threatened/Endangered Wildlife

IA

Geologic Value

Historic Value

other Value

Recreation Value.

Scenic Value

0.90 Federal Land code 103

0.90 Federal Iand Code 104

0.90 Federal Iand Code 105

0.90 Federal Land Code 106

0.90 Federal Land Code 107

0.90 Federal Lând Code 108

0.90 Federal Land code 198

\begin{tabular}{|c|c|c|}
\hline & xist & Prob \\
\hline $\begin{array}{l}\text { Fish } \\
\text { wildlife }\end{array}$ & $\mathbf{Y}$ & $\begin{array}{l}0.90 \\
0.90 \\
0.90 \\
0.90 \\
0.75 \\
0.90 \\
0.90 \\
0.90 \\
0.90 \\
0.90\end{array}$ \\
\hline
\end{tabular}


DATE: 11/24/95

PAGE NO: 25

FERC

Number

Plant Name

Stream

State

10749 SAYLORVILLE

DES MOINES $R$

Name

IA

county Name

River Basin

POLK

DES MOINES RIVER BASIN

Class Owner Name

$R$ IOWA HYDRO DEV CORP

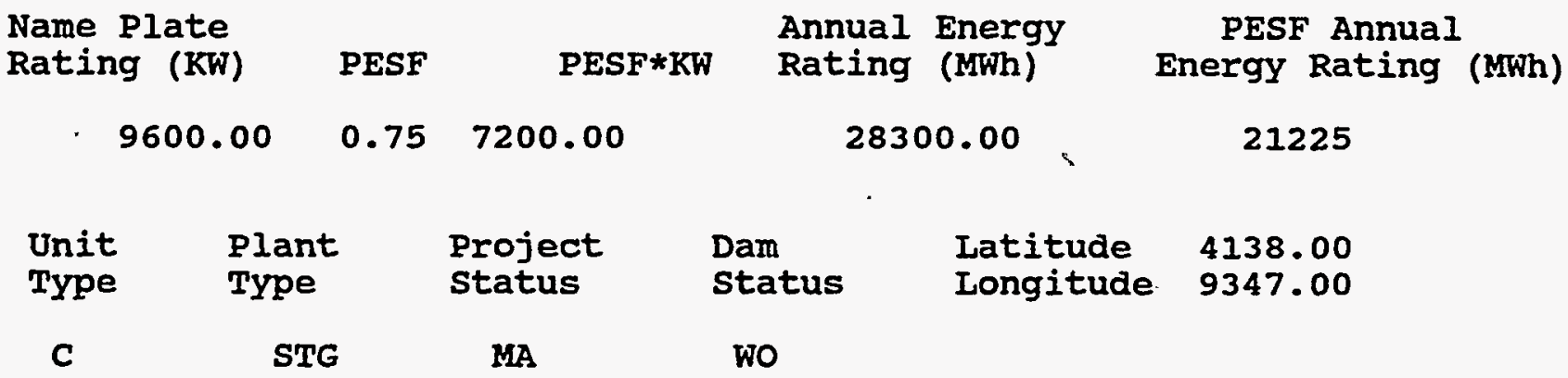

Factor

Exist Prob

Factor

Exist Prob

Wild/Scenic Protection

wild/Scenic Tributary or

Upstream/Downstream

wild/Scenic Location

Cultural Value

Fish Presence Value

Geologic Value

Historic Value

other Value

Recreation.Value

Scenic Value

\section{$\longrightarrow$}

0.90

0.90

0.90

0.90

0.90

0.90

0.90

0.90

0.90

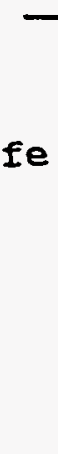

0.90
0.90
0.90
0.90
0.75
0.90
0.90
0.90
0.90
0.90


FERC

Number

11174

RED ROCK

county Name

MARION

$$
\text { Class }
$$

Owner Name

R ELY, TOWN OF

Name Plate
Rating (KW)

PESF

PESF*KW

Annual Energy

30000.00

0.7522500 .00

110000.00

stream

state

Name

IA

River Basin

DES MOINES RIVER BASIN

PESF Annual

$\begin{array}{cccccc}\text { Unit } & \text { Plant } & \text { Project } & \text { Dam } & \text { Latitude } & 4122.00 \\ \text { Type } & \text { Type } & \begin{array}{c}\text { Status } \\ \text { Status }\end{array} & \begin{array}{l}\text { Longitude } \\ 9259.00\end{array} \\ \text { C } & \text { ROR } & \text { Po } & \text { wo } & & \end{array}$

Factor

Exist Prob

Factor

Exist Prob

Wild/Scenic Protection

wild/Scenic Tributary or Upstream/Downstream wild/Scenic Location

0.90 Wildife Value 0.90 Threatened/Endangered Fish Threatened/Endangered Wildlife

0.90 Cultural Value

0.90 Federal Land Code 103
$\begin{array}{lll}0.90 & \text { Federal Land Code } 104 \\ 0.90 & \text { Federal Land Code } 105\end{array}$
0.90

$\begin{array}{lll}0.90 & \text { Federal Land Code } 105 \\ 0.90 & \text { Federal Land Code } 106 \\ 0.90 & \text { Federal Land Code } 107\end{array}$

.90

Fish Presence Value

0.90

Federal Land Code 107
Federal Land Code 108

$\begin{array}{lll}0.90 & \text { Federal Land Code } 108 \\ 0.90 & \text { Federal Land Code } 198\end{array}$

Gistoric Value

0.75

0.90

0.90

0.90

0.90

Recreation Value

0.90

0.90 
FERC Number

11245
Plant Name MISSISSIPPI L\&D 9

County Name ALIAMAKEE

\section{Stream}

MISSISSIPPI R

River Basin

WISCONSIN RIVER BASIN
State

Name

IA

\section{Class Owner Name}

R IOWA HYDROPOWER DEV CORP

Name Plate

Rating (KW)

PESF

Annual Energy PESF*KW

$$
\text { Rating (MWh) }
$$

PESF Annual Energy Rating (MWh)

$$
\begin{array}{lllll}
10000.00 & 0.50 & 5000.00 & 85000.00 & 42500
\end{array}
$$

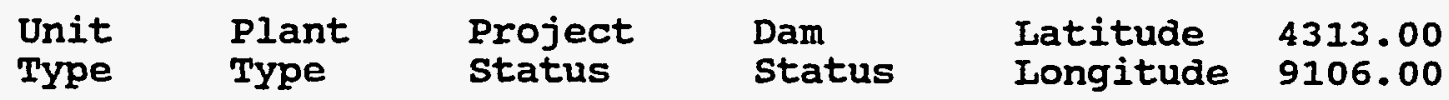

C ROR $\mathrm{XX}$ WO

Factor

Wild/Scenic Protection Wild/Scenic Tributary or Upstream/Downstream wild/scenic Location Cultural value Fish Presence Value Geologic Value Historic Value other Value Recreation Value Scenic Value
Exist Prob

\begin{tabular}{|c|c|c|}
\hline & $Y$ & 0.75 \\
\hline $\begin{array}{l}\text { Fish } \\
\text { wildlife }\end{array}$ & & $\begin{array}{l}0.90 \\
0.90 \\
0.90\end{array}$ \\
\hline & $Y$ & $\begin{array}{l}0.75 \\
0.90 \\
0.90 \\
0.90 \\
0.90 \\
0.90\end{array}$ \\
\hline
\end{tabular}

Factor
Exist Prob 
DATE: $11 / 24 / 95$

PAGE NO: 28

FERC

Number

11246

MISSISSIPPI L\&D 10

County Name

CLAYTON

Class

Owner Name

R IOWA HYDROPOWER DEV CORP

Name Plate

Rating (KW)

PESF

PESF*KW

Annual Energy

Rating (MWh)

95900.00

0.508400 .00

Project status

$\mathrm{XX}$

c

ROR

Exist Prob

Factor

Latitude

Status

Longitude

4247.00

พอ state

Name

IA
PESF Annual

Energy Rating (WWh)

47950
Factor

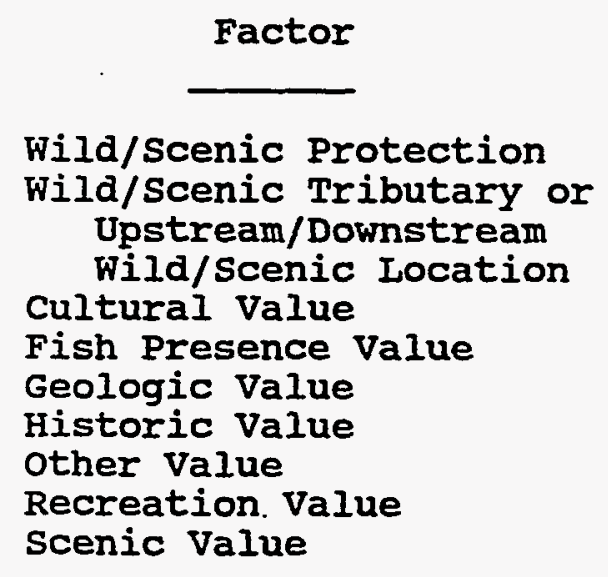

Wild/Scenic Protection ild/Scenic Tributary or Upstream/Downstream Cultural Value

Fish Presence Value

Geologic Value

Historic Value

Recreation. Value

Scenic Value

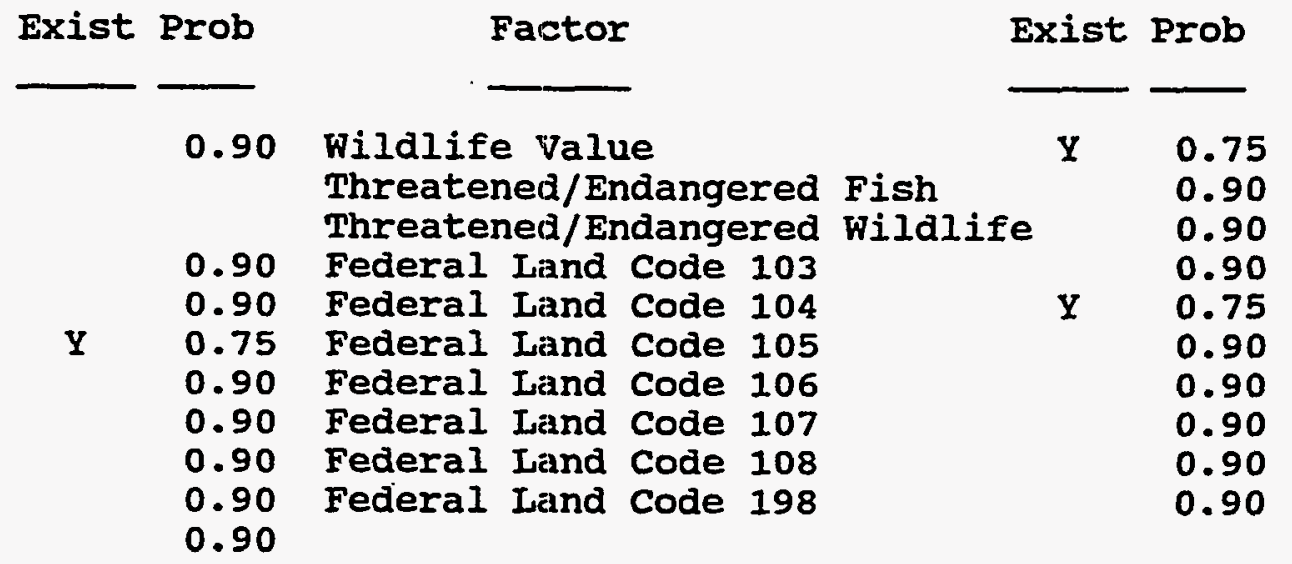


DATE: $11 / 24 / 95$

PAGE NO: 29

FERC

Number

Plant Name

Stream

State

11247 MISSISSIPPI L\&D 11

MISSISSIPPI R

Name

\title{
11247 MISSISSIPPI I\&D 11
}

County Name

River Basin

DUBUQUE

MAQUOKETA RIVER BASIN

\author{
Class Owner Name \\ $M$ IOWA HYDROPOWER DEV CORP
}

\begin{tabular}{|c|c|c|c|c|c|c|}
\hline $\begin{array}{l}\text { Name } \\
\text { Ratin }\end{array}$ & & PESF & PESF*KW & $\begin{array}{l}\text { Annual } \\
\text { Rating }\end{array}$ & $\begin{array}{l}\text { Energy } \\
\text { (MWh) }\end{array}$ & $\begin{array}{l}\text { PESF An } \\
\text { Energy Rat }\end{array}$ \\
\hline & .00 & 0.50 & 7000.00 & 9110 & 10.00 & 45550 \\
\hline $\begin{array}{l}\text { Unit } \\
\text { Type }\end{array}$ & $\begin{array}{l}\text { PI } \\
\text { Tyl }\end{array}$ & & $\begin{array}{l}\text { Project } \\
\text { Status }\end{array}$ & $\begin{array}{l}\text { Dam } \\
\text { status }\end{array}$ & $\begin{array}{l}\text { Latitude } \\
\text { Longitude }\end{array}$ & $\begin{array}{l}4232.00 \\
9038.00\end{array}$ \\
\hline C & & & PO & พอ & & \\
\hline
\end{tabular}

Factor

Exist Prob

Factor

Exist Prob

Wild/Scenic Protection

Wild/Scenic Tributary or Upstream/Downstream wild/Scenic Location

Cultural Value

Fish Presence Value

Geologic Value

Historic Value

other Value

Recreation Value

Scenic Value

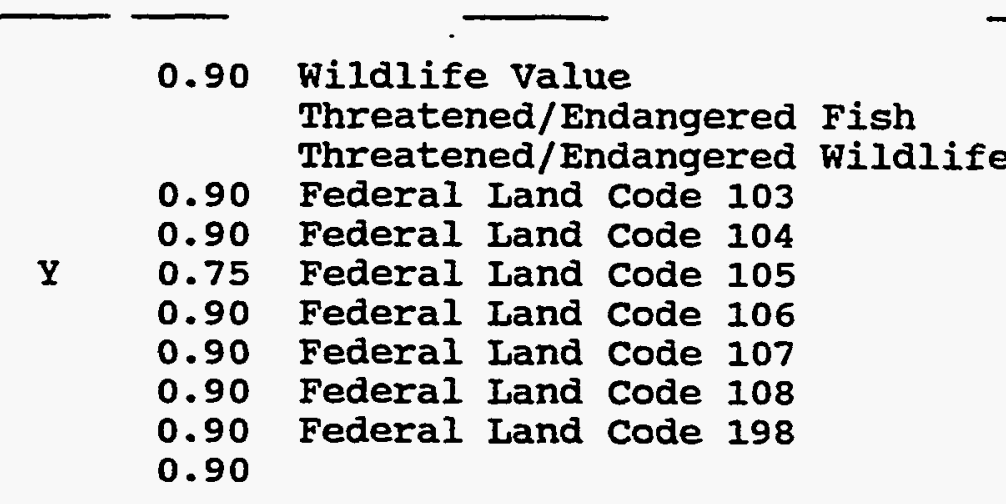

IA 

R E S O U R C E
D A T A B A S E
I I S T N $\mathrm{G}$

DATE: $11 / 24 / 95$

PAGE NO: 30

FERC

Number

Plant Name

11248 MISSISSIPPI I\&D 12

stream

State

Name

MISSISSIPPI $\mathbf{R}$

IA

County Name

JACKSON
River Basin

MAQUOKETA RIVER BASIN

\begin{tabular}{|c|c|c|c|c|c|c|}
\hline \multirow{2}{*}{\multicolumn{3}{|c|}{$\begin{array}{c}\text { Class } \\
\text { M }\end{array}$}} & \multicolumn{2}{|c|}{ Owner Name } & \multirow[b]{2}{*}{ CORP } & \multirow[b]{3}{*}{$\begin{array}{l}\text { PESF Annual } \\
\text { Energy Rating (MWh) }\end{array}$} \\
\hline & & & IOWA HYDROPC & POWER DEV C & & \\
\hline \multicolumn{2}{|c|}{$\begin{array}{l}\text { Name Plate } \\
\text { Rating (KW) }\end{array}$} & PESF & PESF*KW & $\begin{array}{l}\text { Annual } \\
\text { Rating }\end{array}$ & $\begin{array}{l}\text { Energy } \\
\text { (MWh) }\end{array}$ & \\
\hline \multicolumn{2}{|c|}{8200.00} & 0.50 & 4100.00 & \multicolumn{2}{|c|}{36500.00} & 18250 \\
\hline $\begin{array}{l}\text { Unit } \\
\text { Type }\end{array}$ & \multicolumn{2}{|c|}{$\begin{array}{l}\text { Plant } \\
\text { Type }\end{array}$} & $\begin{array}{l}\text { Project } \\
\text { Status }\end{array}$ & $\begin{array}{l}\text { Dam } \\
\text { status }\end{array}$ & $\begin{array}{l}\text { Latitude } \\
\text { Longitude }\end{array}$ & $\begin{array}{l}4216.00 \\
9025.00\end{array}$ \\
\hline C & \multicolumn{2}{|c|}{ ROR } & $\mathrm{XX}$ & \multicolumn{2}{|l|}{ พO } & \\
\hline
\end{tabular}

Factor

Exist Prob

Factor

Exist Prob

Wild/scenic Protection

Wild/Scenic Tributary or

0.90 Wildlife Value

Threatened/Endangered Fish Threatened/Endangered Wildlife

Upstream/Downstream

wild/Scenic Location

Cultural Value

Fish Presence Value

$\begin{array}{ll}0.90 & \text { Federal Irand Code } 103 \\ 0.90 & \text { Federal I and Code } 104\end{array}$

Geologic Value

0.75 Federal Land Code 105

0.90 Federal Land Code 106

0.90 Federal Land Code 107

0.90 Federal Land Code 108

0.90 Federal. Land Code 198

other Value

Recreation value

0.90

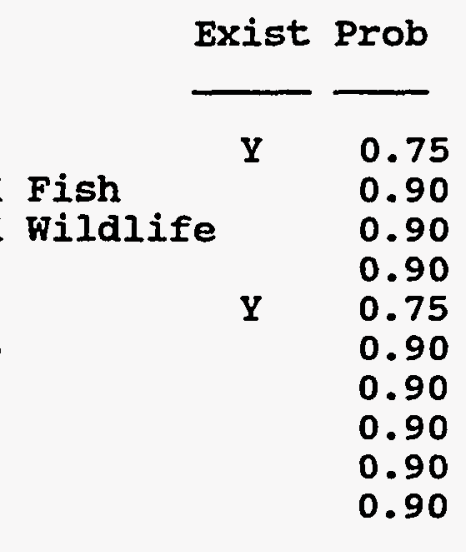


R E S O URCE. D A T A B A S E I I S T I N G

DATE: $11 / 24 / 95$

PAGE NO: 31

FERC

Number

Plant Name

Stream

State

Name

11249 MISSISSIPPI I\&D 13

MISSISSIPPI $R$

IA

County Name

CLINTON

River Basin

MISSISSIPPI MAIN STREAM

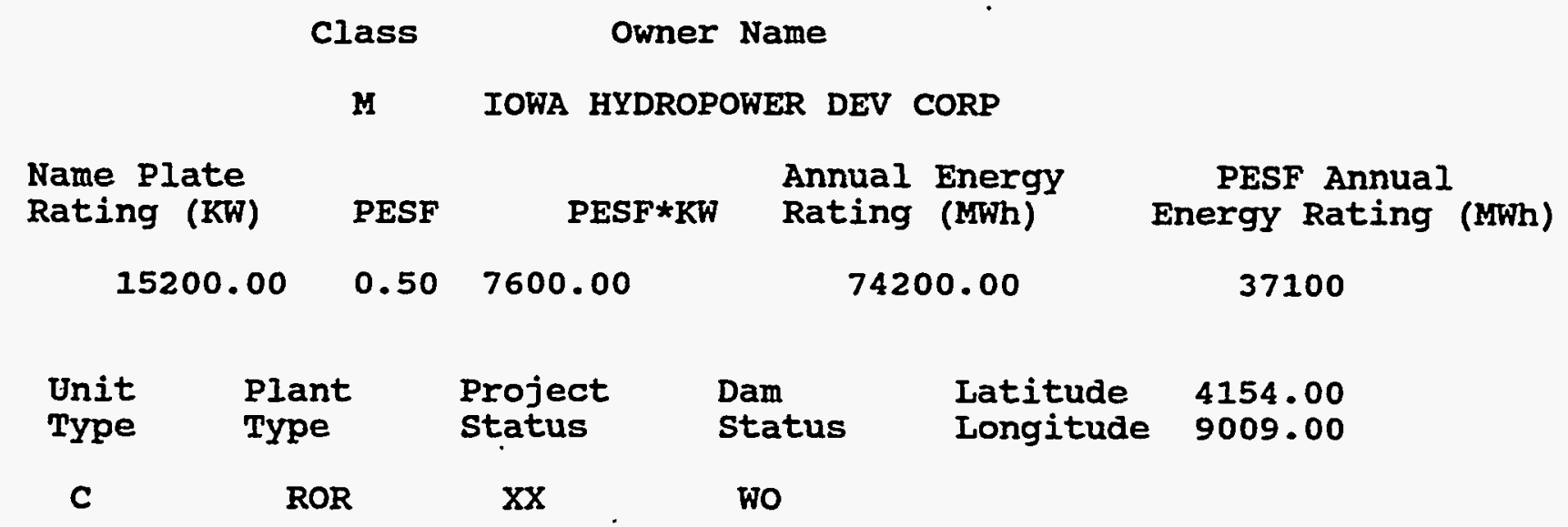

Factor

wild/scenic Protection

Wild/Scenic Tributary or Upstream/Downstream wild/Scenic Location Cultural value Fish Presence Value

Geologic Value Historic Value other Value Recreation value Scenic Value
Exist Prob

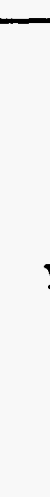

Exist Prob

\section{Factor}

$$
0.90
$$

Wildlife Value Threatened/Endangered Fish Threatened/Endangered Wildlife

0.90 Federal Land Code 103

0.90 Federal Land Code 104

0.75

0.90

0.90

0.90

0.90

0.90

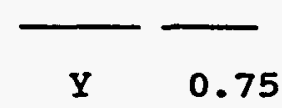

0.90

0.90

0.90

$\mathrm{Y} \quad 0.75$

0.90

0.90

0.90

0.90

0.90 
R E S U R C E D A T A B A S E I I S T I N G

DATE: $11 / 24 / 95$

PAGE NO: 32

FERC

Number

Plant Name

stream

State

11250

MISSISSIPPI I\&D 16

MISSISSIPPI $\mathrm{R}$

Name

IA

County Name

River Basin

MUSCATINE

IOWA RIVER BASIN

$\begin{array}{cc}\text { Class } & \text { Owner Name } \\ R & \text { IOWA HYDROPOWER DEV CORP }\end{array}$

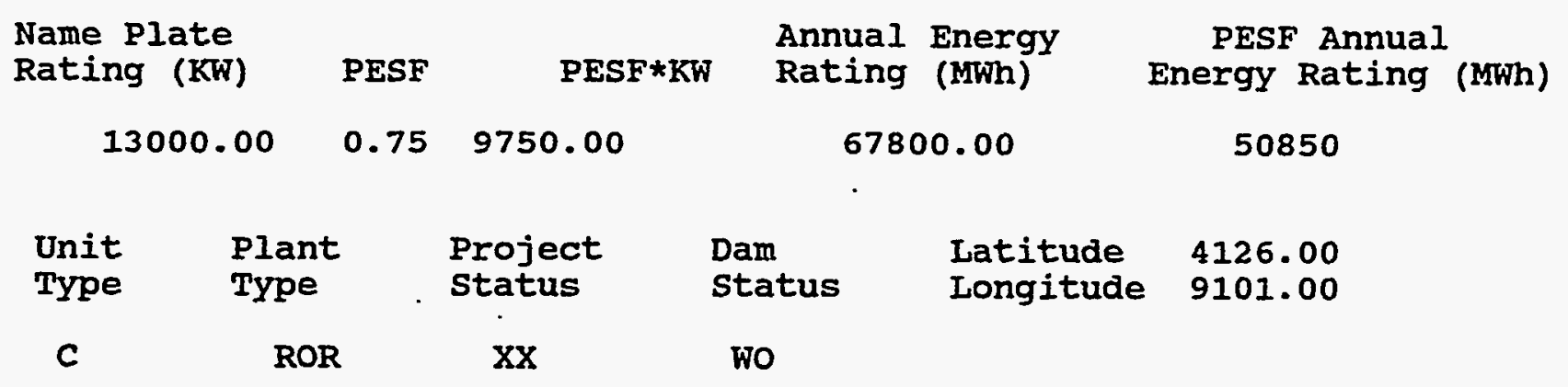

Factor

Wild/Scenic Protection

Wild/Scenic Tributary or

Upstream/Downstream

wild/Scenic Iocation

Cultural value

Fish Presence Value

Geologic Value

Historic Value

other Value

Recreation Value

Scenic Value
Exist Prob

Factor

Exist Prob

0.90

wildlife Value

Threatened/Endangered Fish

Threatened/Endangered Wildlife

0.90 Federal Iand code 103

0.90 Federal Iand Code 104

0.90 Federal Iand Code 105

0.90 Federal Land Code 106

0.90 Federal Iand Code 107

0.90 Federal Land Code 108

0.90 Federal Iand Code 198 
FERC

Number

11251
Plant Name

MISSISSIPPI L\&D 18

County Name

DES MOINES stream

MISSISSIPPI R

River Basin

MISSISSIPPI MAIN STREAM
State

Name

IA

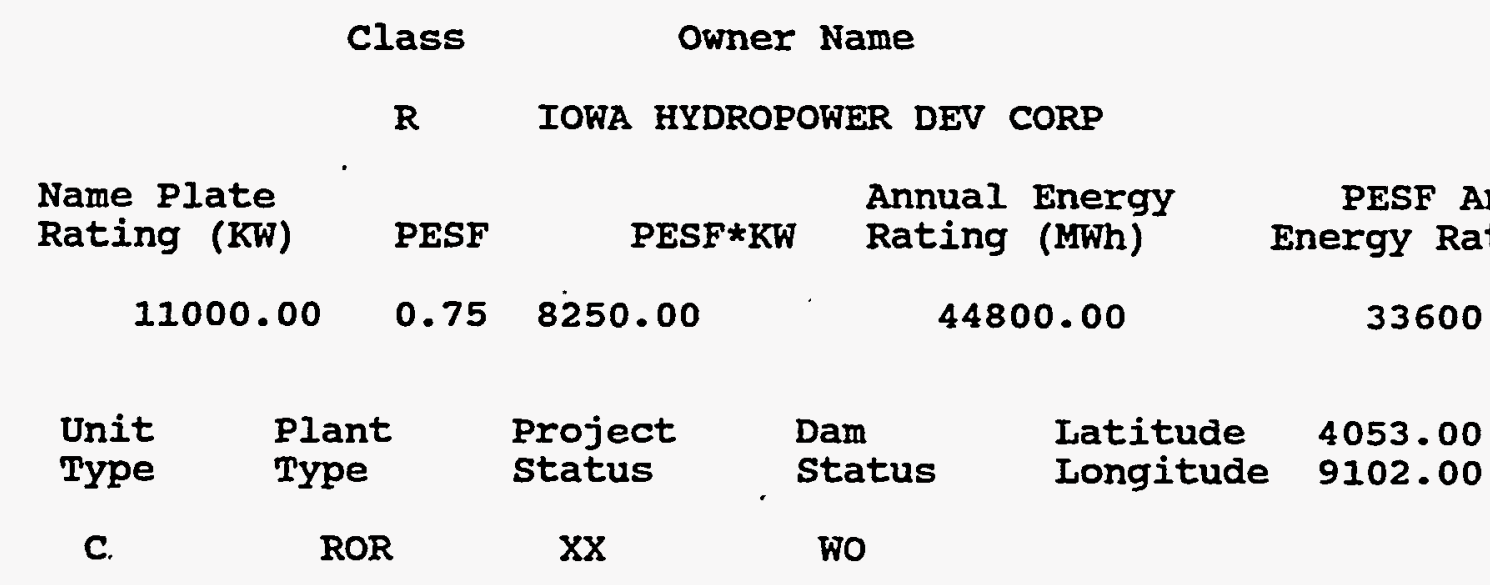

Factor

Exist Prob

Factor

Exist Prob

Wild/Scenic Protection

Wild/Scenic Tributary or Upstream/Downstream wild/Scenic Location Cultural value Fish Presence Value Geologic Value Historic Value other Value Recreation Value Scenic Value

\subsection{Wildlife Value} Threatened/Endangered Fish Threatened/Endangered Wildife

0.90 Federal Land Code 103

0.90 Federal Iand Code 104

0.90 Federal Land Code 105

0.90 Federal Iand Code 106

0.90 Federal Land Code 107

0.90 Federal Land Code 108

0.90 Federal Land Code 198 
DATE: $11 / 24 / 95$

PAGE NO: 34

FERC

Number

Plant Name

stream

State

11278 L\&D 15

MISSISSIPPI $\mathbf{R}$

Name

\section{County Name}

SCOTT

River Basin

MISSISSIPPI MAIN STREAM

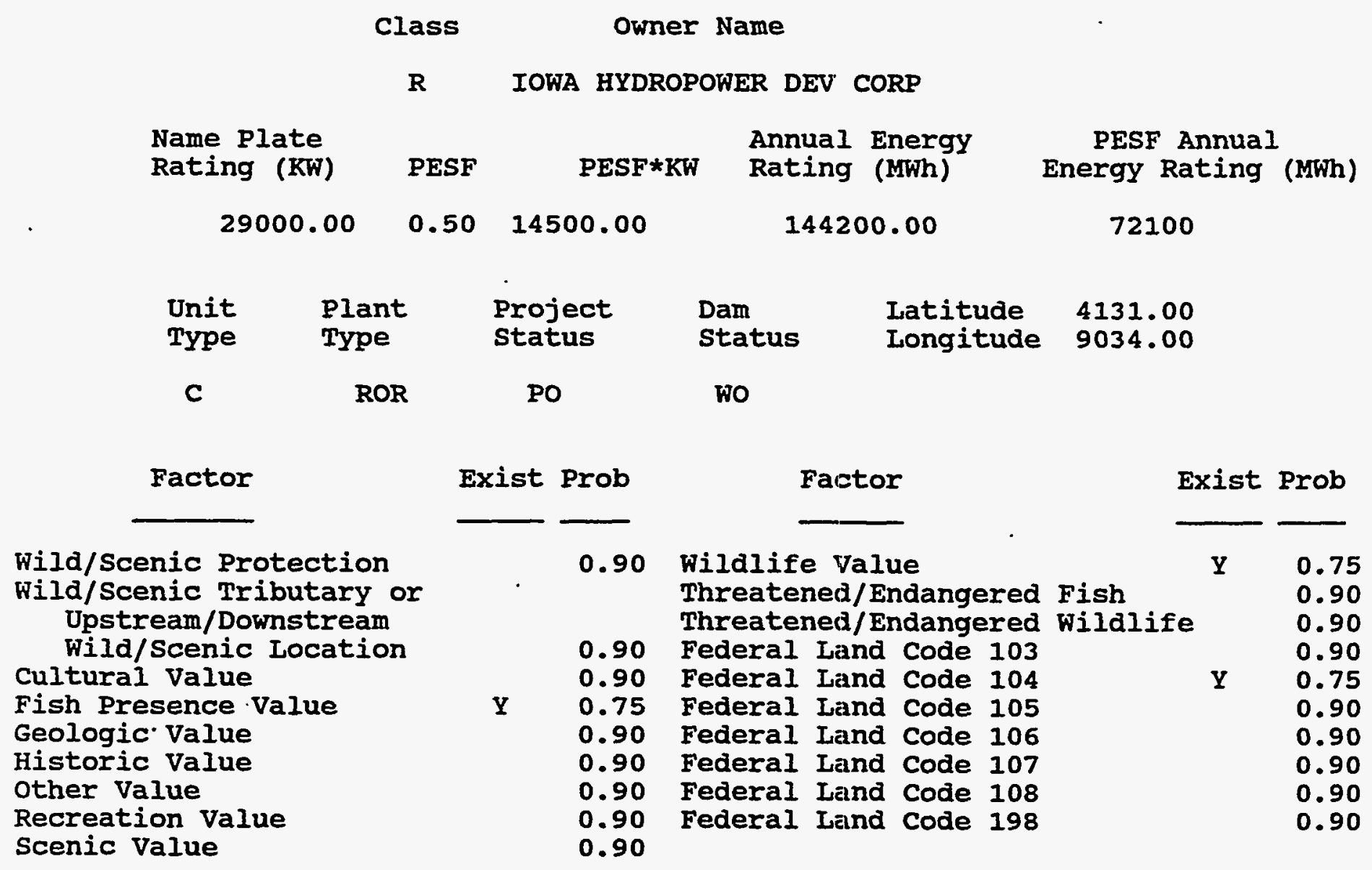


DATE: $11 / 24 / 95$

PAGE NO: 35

FERC

Number

Plant Name
CEDAR R

stream

State

Name

IA
County Name

CHICKASAW \& FLOYD
River Basin

IOWA RIVER BASIN

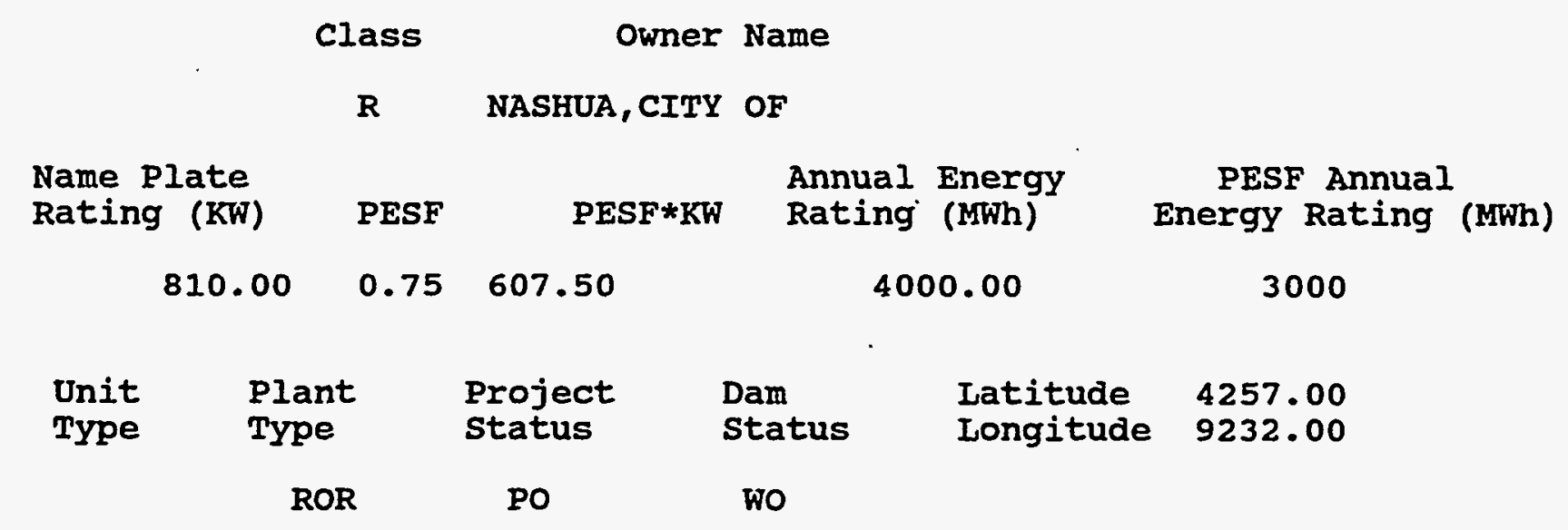

Factor

Exist Prob

Factor

Exist Prob

Wild/Scenic Protection

wild/Scenic Tributary or Upstream/Downstream wild/scenic Location

Y

0.90 Wildlife Value Threatened/Endangered Fish Threatened/Endangered Wildlife

0.90

$\begin{array}{ll}0.90 & \text { Federal Land Code } 103 \\ 0.90 & \text { Federal Land Code } 104\end{array}$

0.90

0.90

Federal Land Code 104

0.90

0.75

Federal Land Code 105

0.90

0.90 Federal Land Code 106

0.90 Federal Land Code 107

0.90 Federal Land Code 108

0.90

Fish Presence Value

Geologic Value

0.90

Federal. Land Code 198

0.90

0.90

0.90

0.90

0.90

0.90 
R E S O U R C E.

DATE: $11 / 24 / 95$
D A T A B A S E

FERC

Number

11342
Plant Name

MONTICELLO MILI

County Name

JONES
PAGE NO: 36
I I S T I N G

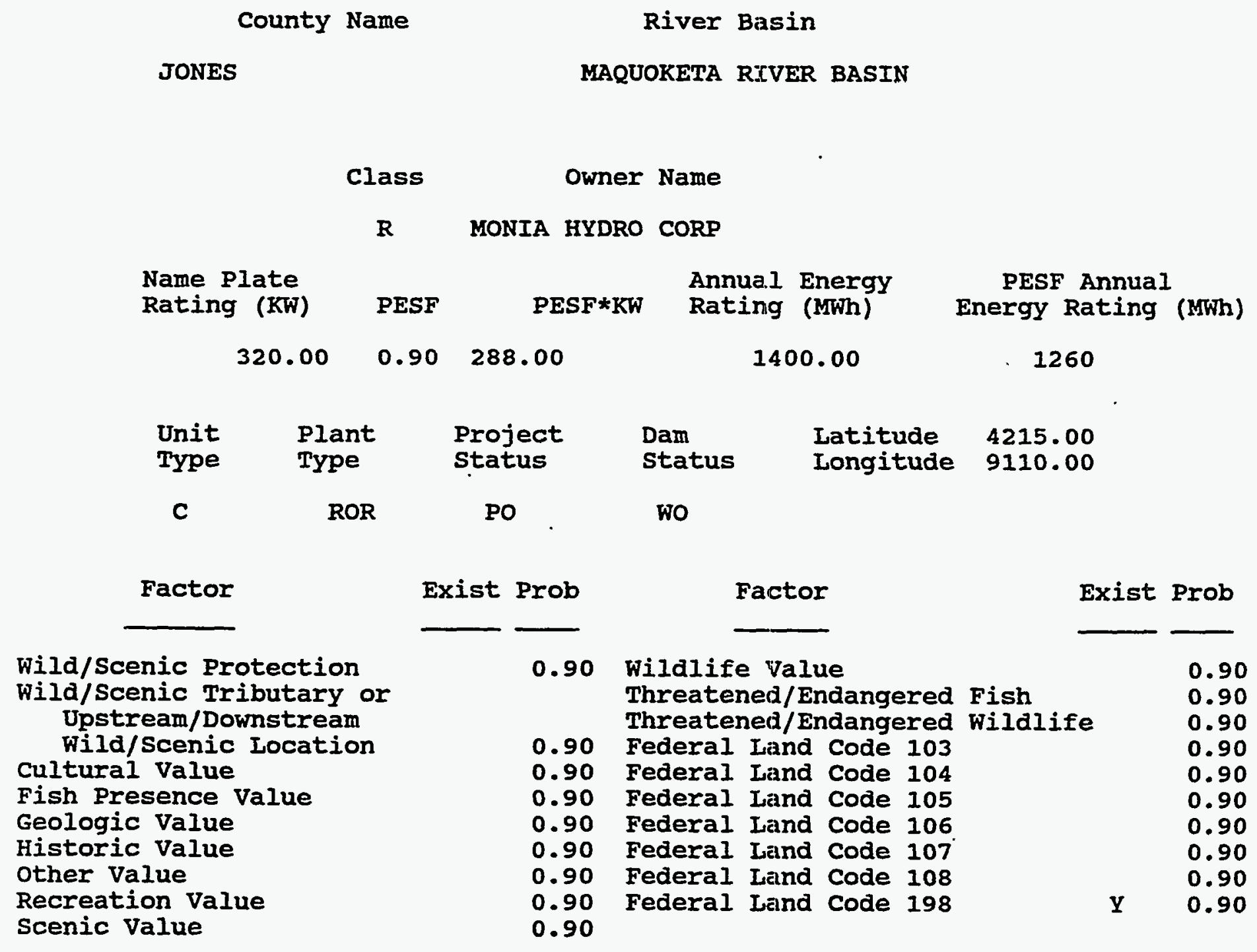

state

Name

IA
MAQUOKETA R

stream
Factor

Wild/Scenic Protection ild/Scenic Tributary or Upstream/Downstream wild/Scenic Location Fish Presence Value other Value Recreation Value Scenic Value
Exist Prob
0.90
0.90
0.90
0.90
0.90
0.90
0.90

0.90

0.90

-190
0.90

0.90

0.90

0.90

0.90

Federal Land Code 108

$\mathbf{Y}$ 0.90 
DATE: $11 / 24 / 95$

FERC

Number

Plant Name

11343 RUTLAND MILL

Factor

Exist Prob

Factor

Exist Prob

Wild/Scenic Protection

Wild/Scenic Tributary or

Upstream/Downstream

wild/Scenic Location

Cultural value

Fish Presence Value

Geologic Value

Historic Value

other Value

Recreation Value

Scenic Value
PAGE NO: 37

State

Name

IA
County Name

HUMBOLDT

Class Owner Name

R RUTIA HYDRO CORP

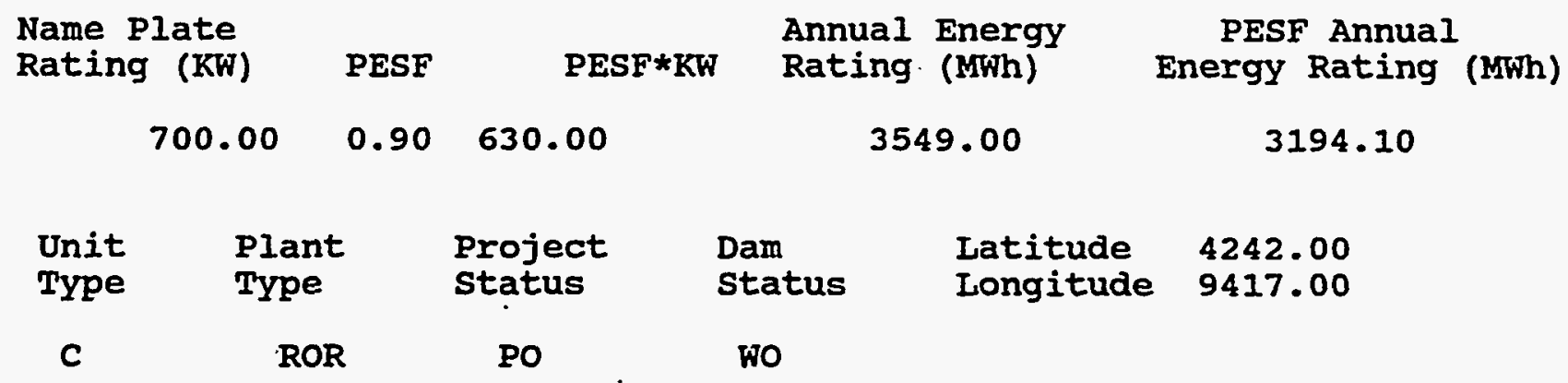

DES MOINES RIVER BASIN

W FK DES MOINES R

River Basin

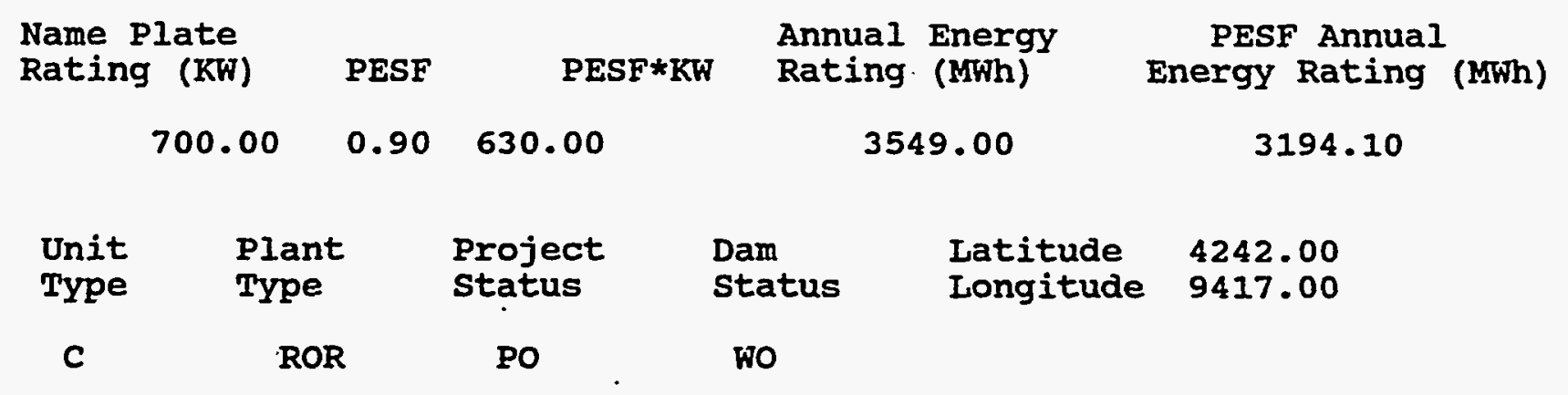

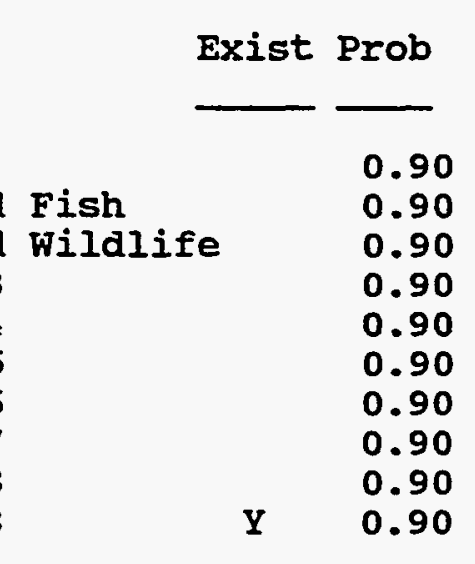


DATE: $11 / 24 / 95$

PAGE NO: 38

FERC

Number

Plant Name

stream

State

11344 HUMBOLDT MILI

W FK DES MOINES $R$

Name

County Name

River Basin

HUMBOLDT

DES MOINES RIVER BASIN

$\begin{array}{cr}\text { Class } & \text { Owner Name } \\ \text { R } & \text { HUMIA HYDRO CORP }\end{array}$

\begin{tabular}{|c|c|c|c|c|c|c|}
\hline \multicolumn{2}{|c|}{$\begin{array}{l}\text { Name Plate } \\
\text { Rating (KW) }\end{array}$} & PESF & PESF*KW & $\begin{array}{l}\text { Annt } \\
\text { Rat }\end{array}$ & $\begin{array}{l}\text { Energy } \\
\text { (MWh) }\end{array}$ & $\begin{array}{l}\text { PESF Ar } \\
\text { ergy Rat }\end{array}$ \\
\hline \multicolumn{2}{|c|}{820.00} & 0.90 & 738.00 & \multicolumn{2}{|c|}{3590.00} & 3231 \\
\hline $\begin{array}{l}\text { Unit } \\
\text { Type }\end{array}$ & \multicolumn{2}{|c|}{$\begin{array}{l}\text { Plant } \\
\text { Pype }\end{array}$} & $\begin{array}{l}\text { Project } \\
\text { status }\end{array}$ & $\begin{array}{l}\text { Dam } \\
\text { status }\end{array}$ & $\begin{array}{l}\text { Latitu } \\
\text { Longit }\end{array}$ & $\begin{array}{l}4244.00 \\
9414.00\end{array}$ \\
\hline C & \multicolumn{2}{|c|}{ ROR } & PO & \multicolumn{2}{|l|}{ wo } & \\
\hline
\end{tabular}

Factor

Exist Prob

Factor

Exist Prob

Wild/Scenic Protection

wild/Scenic Tributary or Upstream/Downstream wild/Scenic Location cultural Value Fish Presence Value Geologic Value Historic Value other value Recreation Value Scenic Value
Exist

0.90

Wilalife Value

0.90 Federal Land Code 103

0.90 Federal Land Code 104

0.90 Federal Land Code 105

0.90 Federal Land Code 106

0.90 Federal Land Code 107

0.90 Federal Land Code 108

0.90 Federal Land Code 198 Threatened/Endangered Wildlife
IA 
DATE: $11 / 24 / 95$

FERC

Number

Plant Name

11346 FORT DODGE MILL

county Name

WEBSTER

Class

Owner Name

P FORIA HYDRO CORPORATION

Name Plate Rating (KW)

PESF

PESF*KW

Annual Energy

Rating (MWh)

$$
320.00
$$

PAGE NO: 39

$$
690.00
$$

0.90

621.00

$$
\begin{gathered}
\text { Plant } \\
\text { Type } \\
\text { ROR }
\end{gathered}
$$

Type

Factor

Exist Prob

Factor

Exist Prob

Wild/Scenic Protection

Wild/Scenic Tributary or

Upstream/Downstream wild/Scenic Location

Cultural value

Fish Presence Value

Geologic Value

Historic Value other Value

Recreation Value

Scenic Value

Project
Status

MA
State

Name

IA
PESF Annual

Energy Rating (MWh)

\section{8}

$\begin{array}{lll}\text { Dam } & \text { Latitude } & 4229.00 \\ \text { Status } & \text { Longitude } & 9411.00\end{array}$

พอ

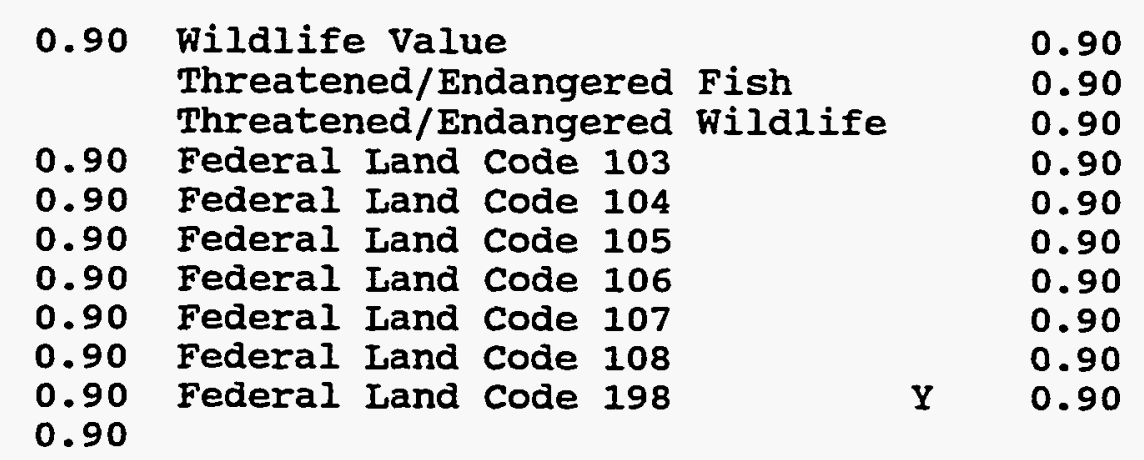


DATE: $11 / 24 / 95$

PAGE NO: 40

FERC

Number

11347

MARBLE ROCK MILL

FIOYD
County Name

Plant Name

\section{Class Owner Name \\ R MARIA HYDRO CORP}

Name plate

Rating (KW)

660.00

PESF

$0.90 \quad 594.00$ state

Name

IA
River Basin

IOWA RIVER EBASIN

\begin{tabular}{|c|c|c|c|c|c|}
\hline $\begin{array}{l}\text { Unit } \\
\text { Type }\end{array}$ & $\begin{array}{l}\text { Plant } \\
\text { Type }\end{array}$ & $\begin{array}{l}\text { Project } \\
\text { status }\end{array}$ & $\begin{array}{l}\text { Dam } \\
\text { status }\end{array}$ & $\begin{array}{l}\text { Latitude } \\
\text { Longitude }\end{array}$ & $\begin{array}{l}4258.00 \\
9253.00\end{array}$ \\
\hline C & ROR & PO & พ० & & \\
\hline
\end{tabular}

\section{Factor}

Wild/Scenic Protection Wild/Scenic Tributary or Upstream/Downstream wild/Scenic Location Cultural Value Fish Presence Value Geologic Value Historic Value other Value Recreation Value Scenic Value
Annual Energy Rating (MWh)
Exist Prob

Factor

Exist Prob

$\begin{array}{ll}0.90 & \text { Wildlife Value } \\ & \text { Threatened/Endangered Fish } \\ & \text { Threatened/Endangered Wildlife } \\ 0.90 & \text { Federal Land Code } 103 \\ 0.90 & \text { Federal Land Code } 104 \\ 0.90 & \text { Federal Land Code } 105 \\ 0.90 & \text { Federal Land Code } 106 \\ 0.90 & \text { Federal Land Code } 107 \\ 0.90 & \text { Federal Land Code } 108 \\ 0.90 & \text { Federal Land Code } 198 \\ 0.90 & \end{array}$

0.90 Wildlife Value Threatened/Endangered Fish

PESF Annual Energy Rating (MWh) 2601 
DATE: $11 / 24 / 95$

FERC

Number

Plant Name

11349 MITCHELL MILL

County Name

MITCHELL
PAGE NO: 41

State

Name

IA
CEDAR R

$$
\text { River Basin }
$$

IOWA RIVER BASIN

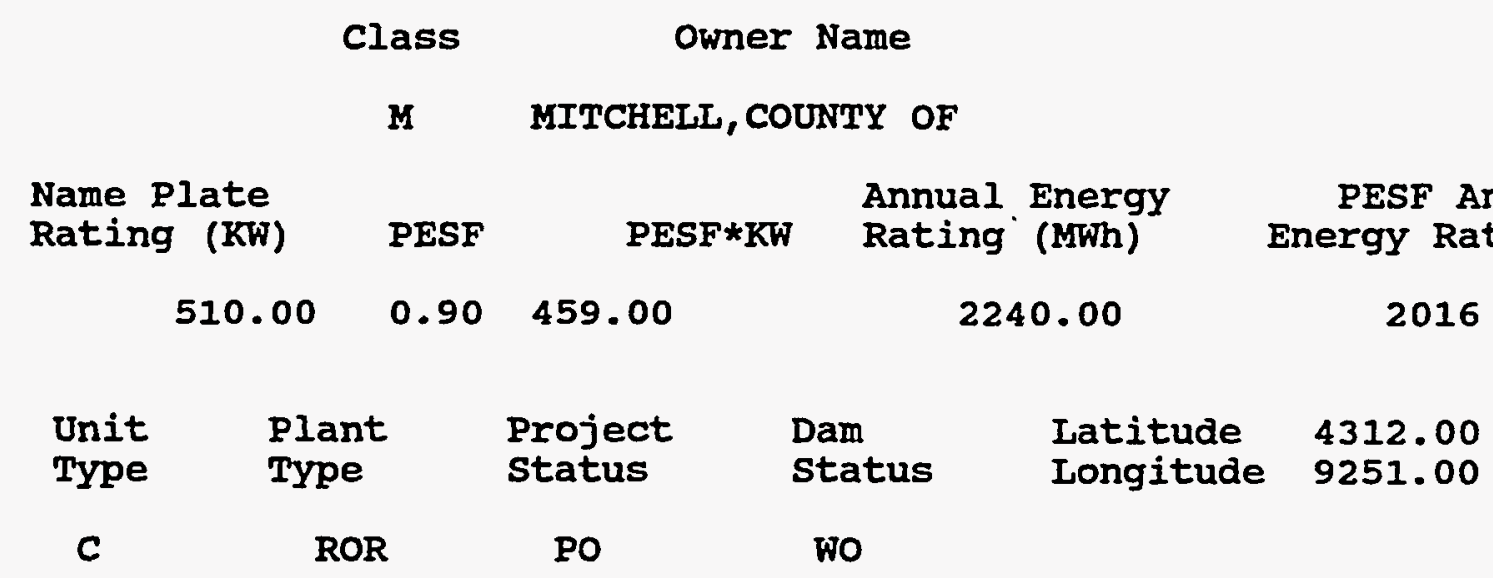

Factor

Exist Prob

Factor

Exist Prob

\subsection{0}

Wildlife Value

Threatened/Endangered Fish

Threatened/Endangered Wildlife

0.90 Federal Land Code 103

0.90 Federal Land Code 104

0.90 Federal Land Code 105

0.90 Federal Land Code 106

0.90 Federal Land Code 107

0.90 Federal Land code 108

0.90 Federal Land Code 198

0.90
0.90

0.90

0.90

0.90

0.90

0.90

0.90

0.90

0.90

Other Value

Recreation Value

Scenic Value 
DATE: $11 / 24 / 95$

FERC

Number

Plant Name

11374 GREENE MILL

County Name

BUTLER

Class Owner Name

M BUTLER, COUNTY OF

Name Plate

Rating (KW)

PESF PESF $\star K W$

Annual Energy

Rating (MWh)

1750.00

0.90333 .00

Project status

$\begin{array}{ll}\text { Unit } & \text { Plant } \\ \text { Type } & \text { Type }\end{array}$

Po

C

ROR

Factor

Exist Prob

Latitude

Longitude

4254.00

Status

พั
State

Name

IA
PESF Annual

Energy Rating (MWh)

1575
Wild/Scenic Protection

Wild/Scenic Tributary or Upstream/Downstream wild/Scenic Location Cultural value Fish Presence Value Geologic Value Historic Value other Value Recreation. Value . Scenic Value
Factor

0.90 Wildlife Value
Threatened/Endangered Fish Threatened/Endangered Wildlife

Exist Prob

0.90

0.90

Federal Land Code 103

Federal Land Code 104

0.90 Federal Land Code 105

0.90 Federal Iand Code 107

0.90 Federal Land Code 108

0.90 Federal Land Code 198

0.90
0.90

0.90

0.90

0.90

0.90

0.90

0.90

0.90

0.90

Y $\quad 0.90$ 
DATE: $11 / 24 / 95$

PAGE NO: 43

FERC

Number

11463
Plant Name

CORALVILLE

county Name

JOHNSON stream

IOWA $\mathbf{R}$

River Basin

IOWA RIVER BASIN
State

Name

IA

$\begin{array}{cc}\text { class } & \text { Owner Name } \\ \text { R } & \text { WHITE HYDROPOWER CO }\end{array}$

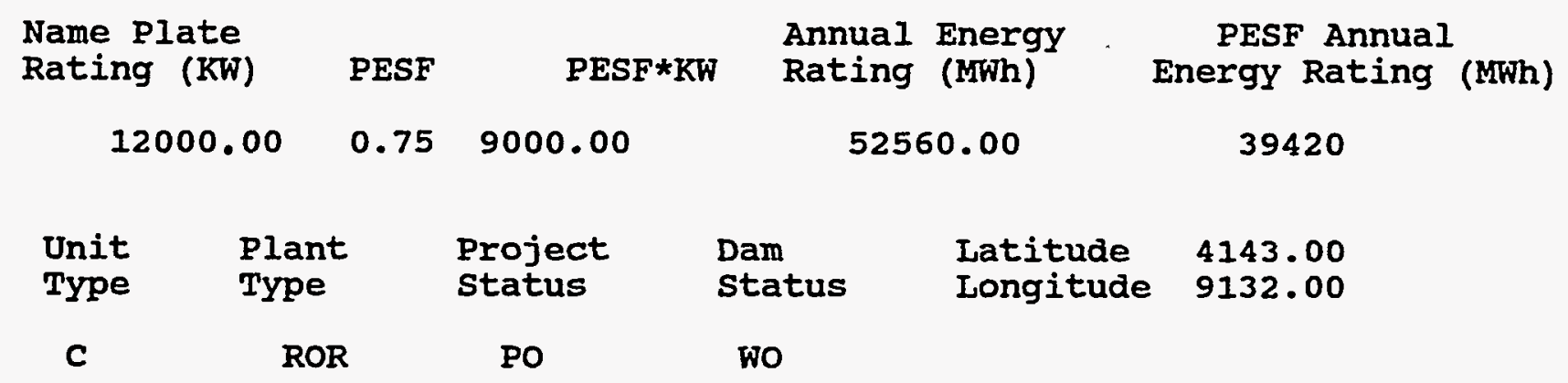

Factor

Exist Prob

Factor

Exist Prob

Wild/Scenic Protection

wild/Scenic Tributary or Upstream/Downstream wild/Scenic Location Cultural value Fish Presence Value Geologic Value Historic Value other value Recreation Value Scenic Value

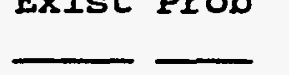

$$
0.90
$$

Wildife value Threatened/Endangered Fish Threatened/Endangered Wildlife 0.90 Federal Land Code 103 0.90 Federal Land Code 104 0.90 Federal Land Code 105 0.90 Federal Land Code 106 0.90 Federal Land Code 107 0.90 Federal Land Code 108 0.90 Federal Land Code 198 0.90 
DATE: $11 / 24 / 95$

PAGE NO: 44

FERC

Number

11485

DELHI MIIL DAM

County Name

DELAWARE

\author{
Class Owner Name \\ R NORTH AMER HYDRO INC
}

Name Plate

Rating (KW)

PESF

Annual Energy

1425.00

$0.90 \quad 1282.50$

PESF*KW

Rating (MWh)

PESF Annual

Energy Rating (NWh)

$$
3300.00
$$

2970 $\begin{array}{ll}\text { Unit } & \text { Plant } \\ \text { Type } & \text { Type }\end{array}$

C

ROR
Project status

PO
Dam Latitude

Status

พอ state

Name

IA
Factor

Wild/Scenic Protection Upstream/Downstream wild/Scenic Location Cultural Value

Fish Presence Value

Geologic'Value

Historic Value

other Value

Recreation Value

Scenic Value
wild/Scenic Tributary or
Exist Prob

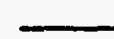

$$
0.90
$$

Wildlife Value Threatened/Endangered Fish Threatened/Endangered Wildlife

Factor

Exist Prob

0.90

Federal Land Code 103

0.90 Federal Land Code 104

0.90 Federal Land Code 105

0.90 Federal Land Code 106

0.90 Federal Iand Code 107

0.90 Federal Land Code 108

0.90 Federal Land Code 198
0.90
0.90

. 0.90

0.90

0.90

0.90

0.90

0.90

0.90

0.90

$Y$

0.90 
DATE: $11 / 24 / 95$

FERC Number

11502 RED ROCK

County Name

MARION

$$
\begin{array}{cr}
\text { Class } & \text { Owner Name } \\
\text { R } & \text { ELY, TOWN OF }
\end{array}
$$

Name Plate

Rating (KW)

PESF

PESF*KW

Annual Energy

30000.00

0.7522500 .00

Rating (NWh)

PESF Annual Energy Rating (MWh)

110000.00

82500
State

Name

IA

\section{Project status}

PA

$\begin{array}{ll}\text { Dam } & \text { Latitude } \\ \text { Status } & \text { Longitude }\end{array}$

4122.00

9259.00
C

ROR
Exist Prob

Factor

Exist Prob

\subsection{0}

Wildlife Value

Threatened/Endangered Fish

Threatened/Endangered Wildlife

0.90 Federal Land Code 103

0.90 Federal Land Code 104

0.90 Federal Land Code 105

0.90 Federal Land Code 106

0.90 Federal Land Code 107

0.90 Federal Land Code 108

0.90 Federal Land Code 198
0.90

0.90

0.90

0.90

$\mathbf{Y}$

0.75

0.90

0.90

0.90

0.90

0.90
Wild/Scenic Tributary or Upstream/Downstream Cultural Value

Fish Presence Value

Geologic Value

Historic Value

other Value

Recreation Value

Scenic Value
0.90 
FERC

Number

IA001
Plant Name

CEDAR RAPIDS

County Name

IINN
River Basin

IOWA RIVER BASIN
State

Name

CEDAR R

IA

$$
\begin{array}{cc}
\text { Class } & \text { Owner Name } \\
\text { M } & \text { CITY OF CEDAR RAPIDS }
\end{array}
$$

\begin{tabular}{|c|c|c|c|c|c|c|}
\hline \multicolumn{2}{|c|}{$\begin{array}{l}\text { Name Plate } \\
\text { Rating (KW) }\end{array}$} & PESF & PESF *KW & \multicolumn{2}{|c|}{$\begin{array}{l}\text { Annual Energy } \\
\text { Rating (MWh) }\end{array}$} & $\begin{array}{l}\text { PESF Annual } \\
\text { Energy Rating (MWh) }\end{array}$ \\
\hline . & 9600.00 & 0.90 & 8640.00 & & 00.00 & 39600 \\
\hline $\begin{array}{l}\text { Unit } \\
\text { Type }\end{array}$ & $\begin{array}{l}\text { Pla } \\
\text { TyE }\end{array}$ & & $\begin{array}{l}\text { Project } \\
\text { Status }\end{array}$ & $\begin{array}{l}\text { Dam } \\
\text { Status }\end{array}$ & $\begin{array}{l}\text { Latitude } \\
\text { Longitude }\end{array}$ & $\begin{array}{l}4157.00 \\
9138.00\end{array}$ \\
\hline c & & & LE & พั & & \\
\hline
\end{tabular}

Factor

Exist Prob

Factor

Exist Prob

Wild/Scenic Protection

Wild/Scenic Tributary or Upstream/Downstream wild/Scenic Location Cultural value

Fish Presence Value

Geologic Value

Historic Value

other Value

Recreation Value

Scenic Value

\subsection{Wildlife Value}

Threatened/Endangered Fish

Threatened/Endangered Wildlife

0.90 Federal Land Code 103

0.90 Federal Liand Code 104

0.90 Federal Land Code 105

0.90 Federal Land Code 106

0.90 Federal Land Code 107

0.90 Federal Land Code 108

0.90 Federal Land Code 198

0.90

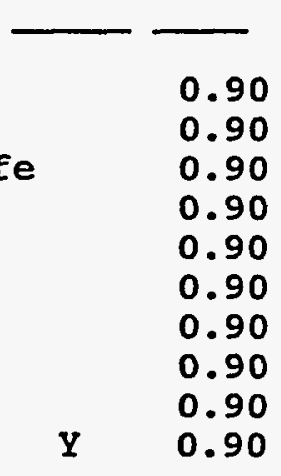


RES O U R C E D A T A B A E L I S T I N G

DATE: $11 / 24 / 95$

PAGE NO: 47

FERC

Number

Plant Name

stream

State

Number

IAOO2 MAQUOKETA

S FK MAQUOKETA $\mathbf{R}$

Name

County Name

River Basin

JACKSON

MAQUOKETA RIVER BASIN

IA

Class Owner Name

P IOWA ELEC IT AND PWR CO

$\begin{array}{lrccc}\begin{array}{l}\text { Name Plate } \\ \text { Rating (KW) }\end{array} & \text { PESF } & \text { PESF*KW } & \begin{array}{c}\text { Annual Energy } \\ \text { Rating (MWh) }\end{array} & \begin{array}{c}\text { PESF Annual } \\ \text { Energy Rating (MWh) }\end{array} \\ 44140.00 & 0.50 & 2070.00 & 8690.00 & 4345\end{array}$

$\begin{array}{llllll}\text { Unit } & \text { Plant } & \text { Project } & \text { Dam } & \text { Latitude } & 4204.00 \\ \text { Type } & \text { Type } & \text { Status } & \text { Status } & \text { Longitude } & 9042.00\end{array}$

C ROR LE W

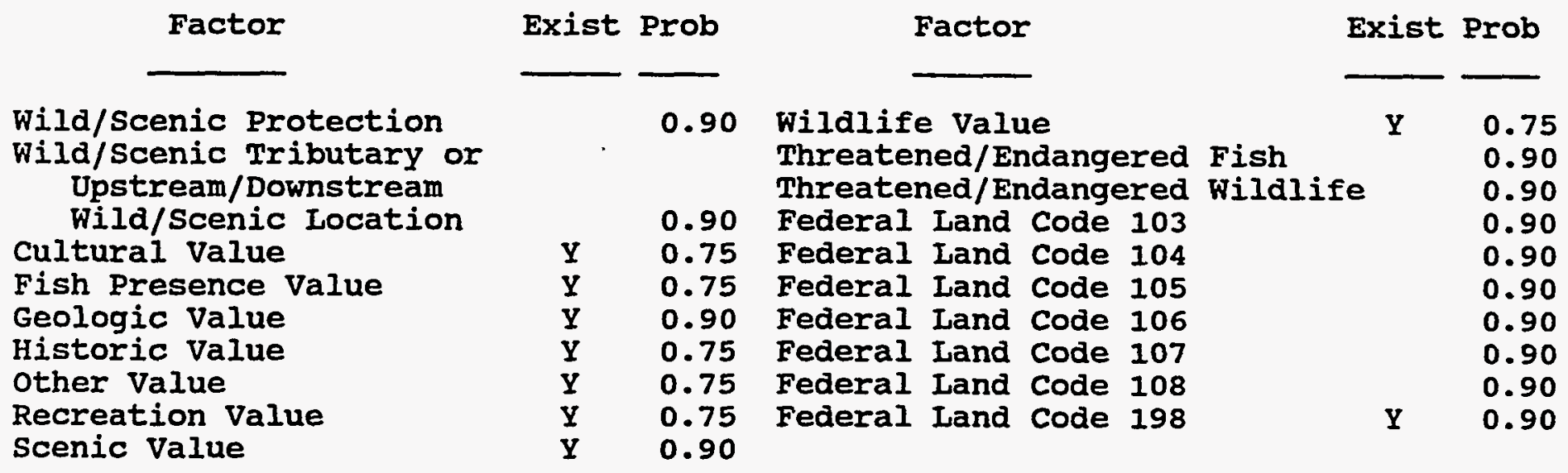


FERC

Number

IA003
Plant Name

MISSISSIPPI L\&D 19

County Name

LEE

\section{stream}

MISSISSIPPI $R$

River Basin

MISSISSIPPI MAIN STREAM
Class Owner Name

F UNION ELECTRIC

Name Plate

Rating (KW)

103831.00

PESF

PESF*KW

Annual Energy

Rating (MWh)

$0.50 \quad 51915.50$

448000.00

PESF Annual

Energy Rating (MWh)

224000

\begin{tabular}{|c|c|c|c|c|c|}
\hline $\begin{array}{l}\text { Unit } \\
\text { Type }\end{array}$ & $\begin{array}{l}\text { Plant } \\
\text { Type }\end{array}$ & $\begin{array}{l}\text { Project } \\
\text { Status }\end{array}$ & $\begin{array}{l}\text { Dam } \\
\text { status }\end{array}$ & $\begin{array}{l}\text { Latitude } \\
\text { Longitude }\end{array}$ & $\begin{array}{l}4023.00 \\
9122.00\end{array}$ \\
\hline C & ROR & IE & $\mathbf{W}$ & & \\
\hline
\end{tabular}

Factor

Exist Prob

Factor

Exist Prob

Wild/Scenic Protection

wild/Scenic Tributary or Upstream/Downstream wild/Scenic Location Cultural value Fish Presence Value

Geologic Value

Historic Value

Other Value

Recreation value

Scenic Value

(1)

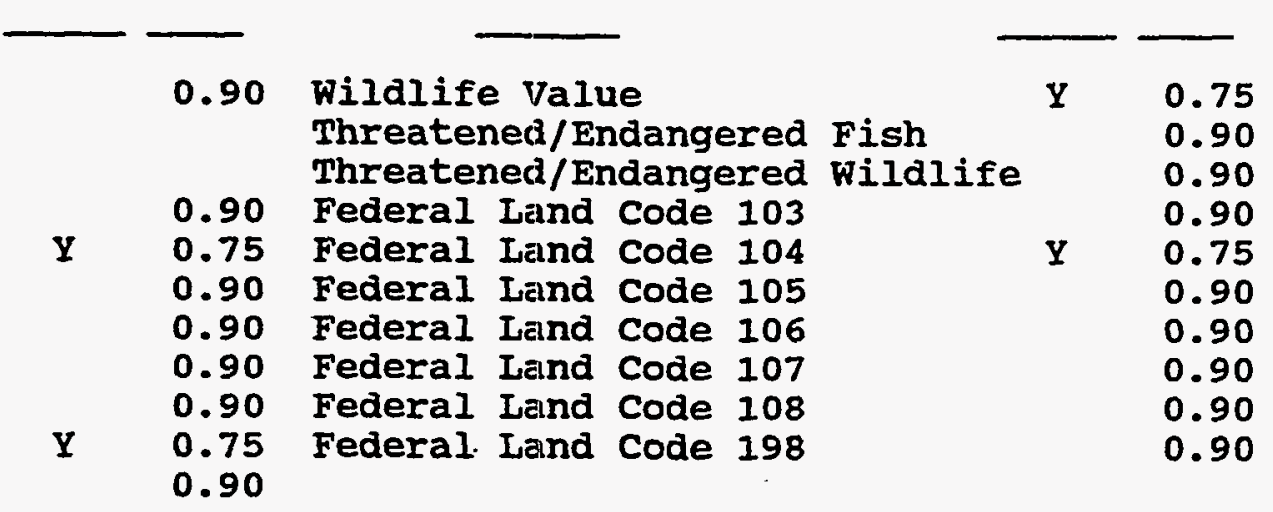


DATE: $11 / 24 / 95$

FERC

Number

Plant Name

IA004 OTTUMWA

County Name

WAPELLO
PAGE NO: 49

state

Name

IA
DES MOINES $R$

River Basin

DES MOINES RIVER BASIN

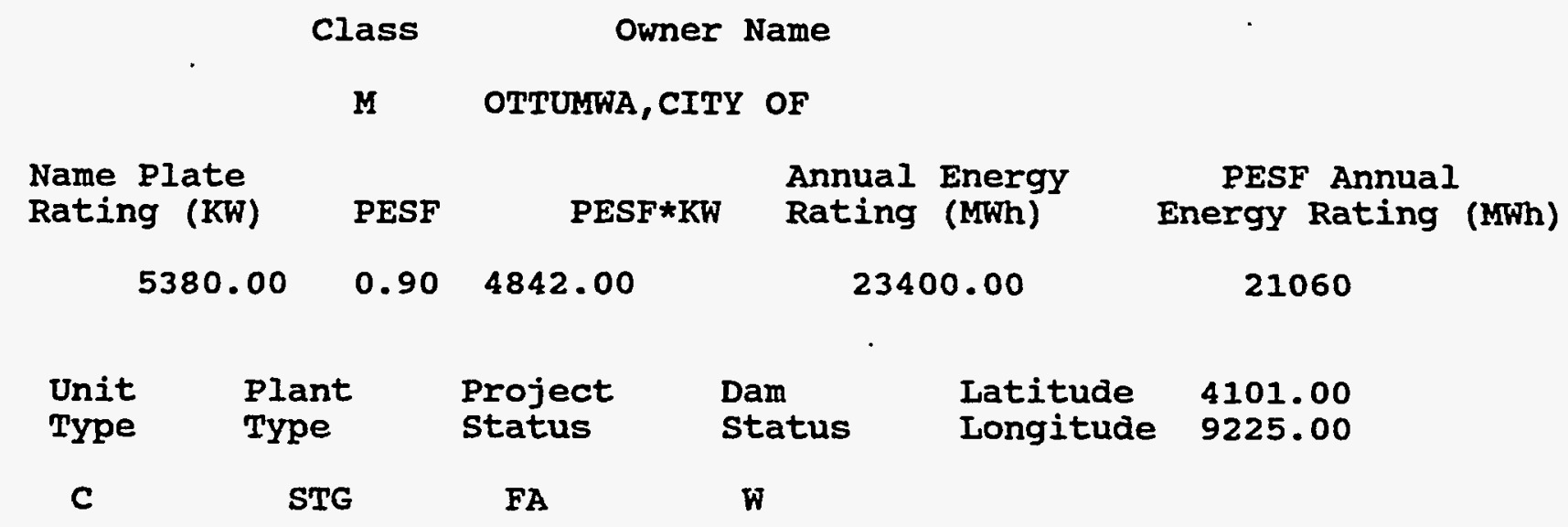

Factor

Exist Prob

$$
0.90
$$

Wild/Scenic Protection Upstream/Downstream wild/Scenic Location Cultural Value Fish Presence Value Geologic Value Historic Value other Value Recreation. Value Scenic Value
Wildlife Value

Threatened/Endangered Fish

Factor

Exist Prob

Threatened/Endangered Wildlife

0.90 Federal Land Code 103

0.90 Federal Land Code 104

0.90 Federal Land Code 105

0.90 Federal Land Code 106

0.90 Federal Land Code 107

0.90 Federal Land Code 108

0.90 Federal Land Code 198

0.90

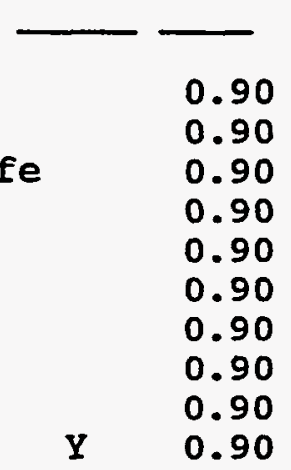


FERC

Number

IAOO5
Plant Name

PANORA (232 IA NO)

County Name

GUIHRIE stream

M RACCOON R
State Name

IA

River Basin

DES MOINES RIVER BASIN

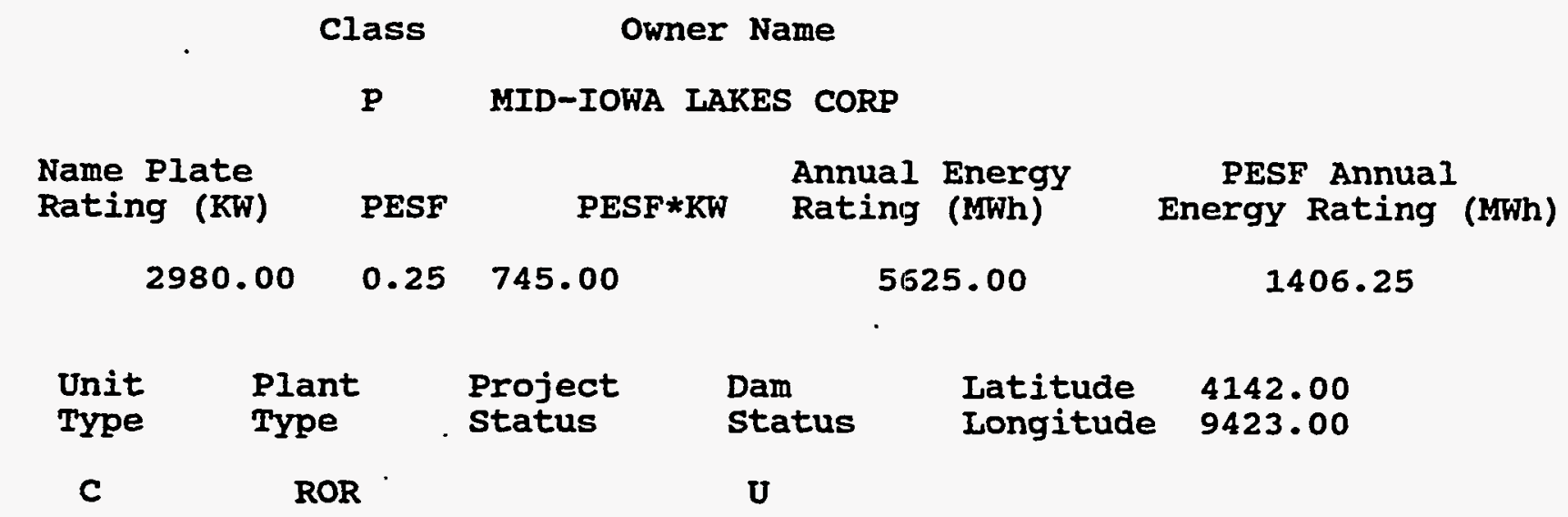

Factor

Wild/Scenic Protection Wild/Scenic Tributary or Upstream/Downstream wild/Scenic Location Cultural Value Fish Presence Value Geologic Value Historic Value other Value Recreation Value Scenic Value
Exist Prob

Factor

Exist Prob

0.90

Wildlife Value Threatened/Endangered Fish Threatened/Endangered Wildlife

0.90 Federal Land Code 103

0.90 Federal Land Code 104

0.90 Federal Land Code 105

0.90 Federal Land Code 106

Y $\quad 0.50$

0.90

0.90

0.90
Federal Land Code 107

Federal Land Code 108

Federal Land Code 198
$\mathbf{Y}$

0.25

0.90

0.90

0.90

0.90

0.90

0.90

0.90

0.90

Y $\quad 0.90$
Exist Prob 
DATE: $11 / 24 / 95$

FERC

Number

Plant Name

IAO06 PROJECT 13 A

county Name

BENTON

Class
PAGE NO: 51

State

Name

CEDAR R

IA

$$
\text { River Basin }
$$

IOWA RIVER BASIN

Owner Name

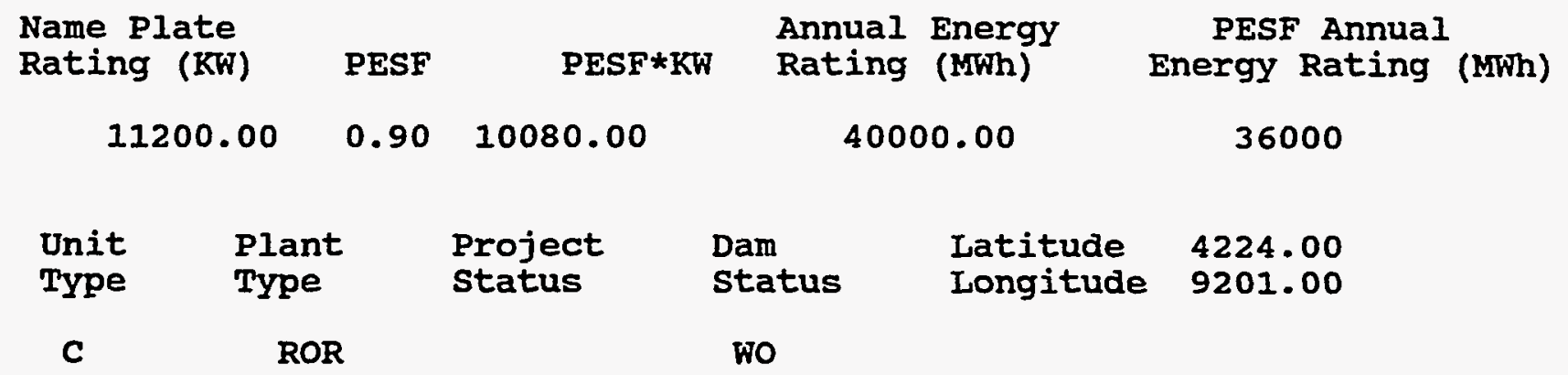

Factor

Exist Prob

Factor

Exist Prob

Wild/Scenic Protection

Wild/Scenic Tributary or

0.90 Wildlife Value
Threatened/Endangered Fish

Threatened/Endangered Wildlife

Exist Prob

Upstream/Downstream

wild/Scenic Location

0.90 Federal Iand code 103

0.90 Federal Land Code 104

0.90

0.90

0.90

0.90

0.90 Federal Land Code 105

0.90

0.90 Federal Land Code 106

0.90

0.90 Federal Land Code 107

0.90

0.90 Federal Land Code 108

0.90

0.90

Federal Land Code 198

0.90

0.90

0.90 
DATE: $11 / 24 / 95$

FERC

Number

Plant Name

IA007 RED ROCK

County Name

MARION

$$
\begin{array}{cc}
\text { Class } & \text { Owner Name } \\
\text { F } & \text { CORPS OF ENGINEERS }
\end{array}
$$

\begin{tabular}{|c|c|c|c|c|c|c|}
\hline $\begin{array}{l}\text { Name } \\
\text { Ratin }\end{array}$ & & PESF & PESF*KT & $\begin{array}{l}\text { Annual } \\
\text { Rating }\end{array}$ & $\begin{array}{l}\text { Energy } \\
\text { (MWh) }\end{array}$ & $\begin{array}{l}\text { PESF Annual } \\
\text { Energy Rating (MWh) }\end{array}$ \\
\hline & .00 & 0.90 & 15480.00 & 10360 & 0.00 & 93240 \\
\hline $\begin{array}{l}\text { Unit } \\
\text { Type }\end{array}$ & $\begin{array}{l}\text { PI } \\
\text { Ty }\end{array}$ & & $\begin{array}{l}\text { Project } \\
\text { Status }\end{array}$ & $\begin{array}{l}\text { Dam } \\
\text { status }\end{array}$ & $\begin{array}{l}\text { Latitude } \\
\text { Longitude }\end{array}$ & $\begin{array}{l}4122.00 \\
9259.00\end{array}$ \\
\hline c & & & & พอ & & \\
\hline
\end{tabular}

Factor

Exist Prob

Factor

Exist Prob

Wild/Scenic Protection

Wild/Scenic Tributary or

Upstream/Downstream

wild/Scenic Location

Cultural Value

Fish Presence Value

Geologic Value

Historic Value

other Value

Recreation Value.

Scenic Value
PAGE NO: 52

State

Name

IA
River Basin

DES MOINES RIVER BASIN 
FERC

Number

IAOOB ROCHESTER

County Name

CEDAR

class

Owner Name
State

Name

CEDAR $\mathbf{R}$

IA

IOWA RIVER BASIN

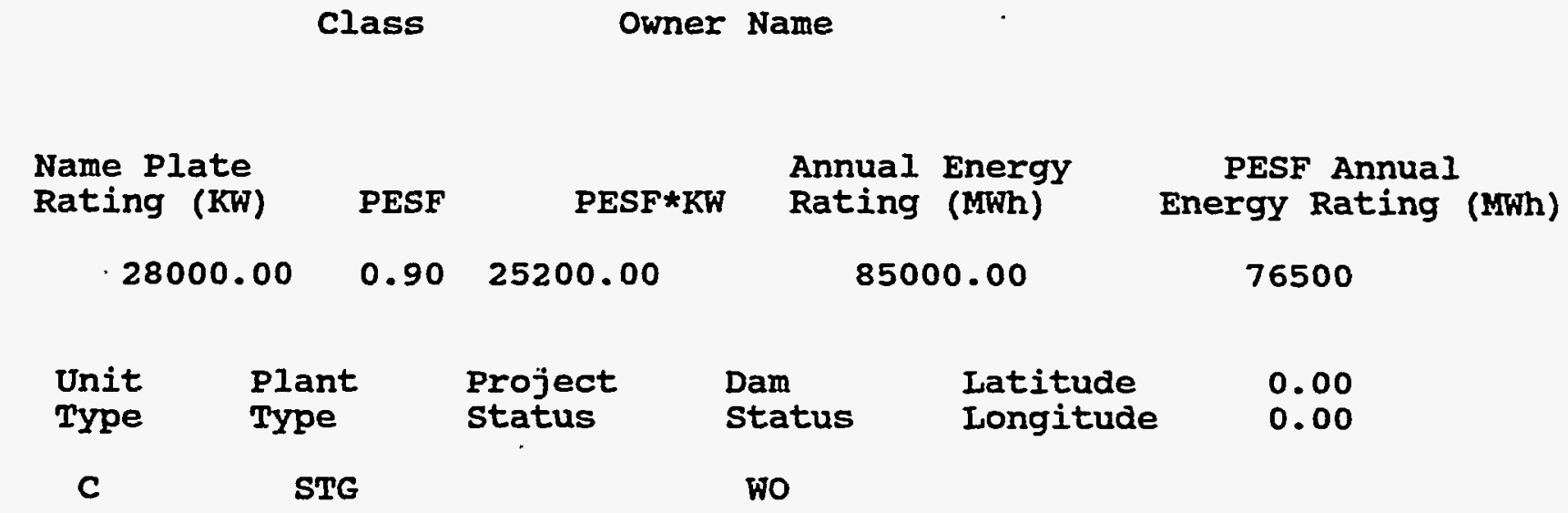

Factor

Exist Prob

Factor

Exist Prob

Wild/Scenic Protection

wild/Scenic Tributary or Upstream/Downstream wild/Scenic Location

0.90 Wildlife Value Threatened/Endangered Fish Threatened/Endangered Wildlife

0.90

$\begin{array}{ll}0.90 & \text { Federal Land Code } 103 \\ 0.90 & \text { Federal Land Code } 104\end{array}$

0.90

Cultural value

Fish Presence Value

0.90

Federal Land Code 105

0.90 Federal Land Code 106

0.90 Federal Land code 107

Historic Value

0.90

Federal Iand Code 108

0.90 Federal Iand code 198

0.90

0.90

0.90

0.90

0.90

0.90

other Value

Recreation Value

0.90

0.90

Scénic Value 
FERC

Number

IA009
Plant Name ST ANSGAR LOWER

County Name MITCHELL

\section{stream}

CEDAR R

River Basin

IOWA RIVER BASIN
State

Name

IA

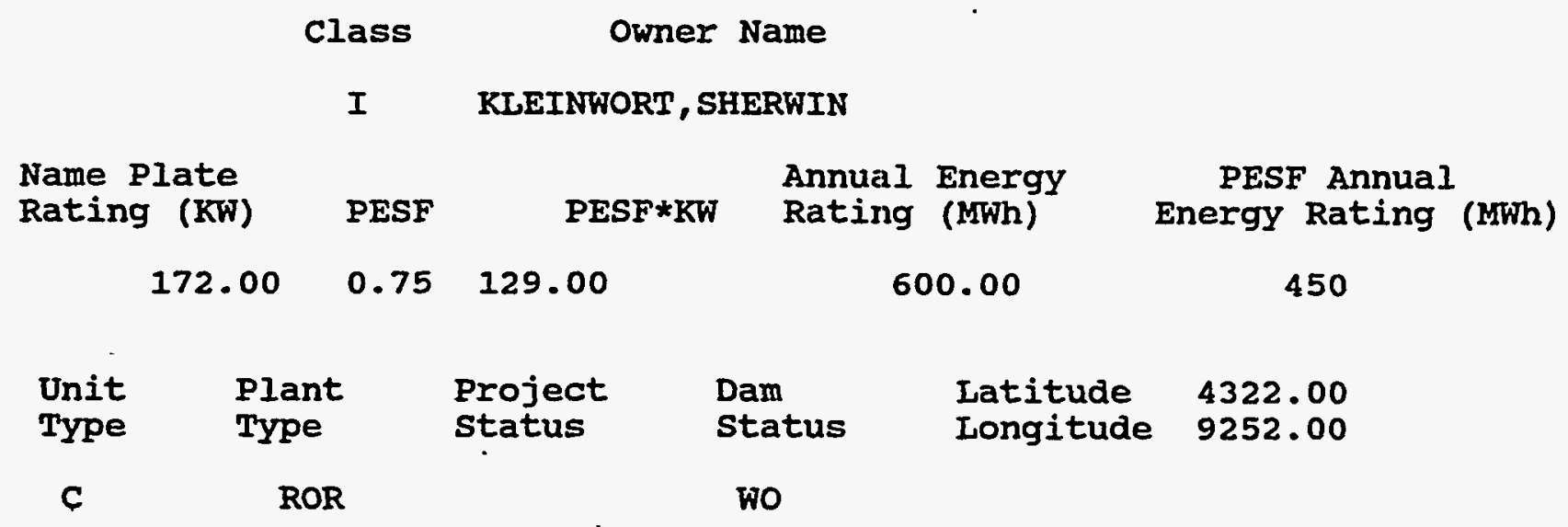

Factor

Wild/Scenic Protection Wild/Scenic Tributary or Upstream/Downstream wild/Scenic Location Cultural.Value Fish Presence Value Geologic Value Historic Value other Value Recreation value Scenic Value
Exist Prob

Factor

Exist Prob

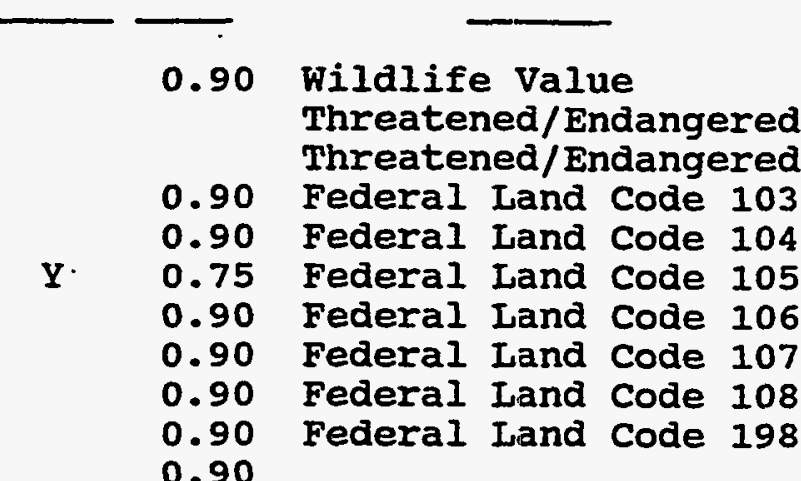

0.90

0.90

0.90

0.90

0.90

0.90

0.90

0.90

0.90

Y $\quad 0.90$ 
DATE: $11 / 24 / 95$

PAGE NO: 55

FERC

Number

Plant Name

Stream

State

IA010 ST ANSGAR UPPER

CEDAR R

Name

County Name

River Basin

MITCHELL

IOWA RIVER BASIN

\section{Class Owner Name \\ P INTERSTATE POWER CO}

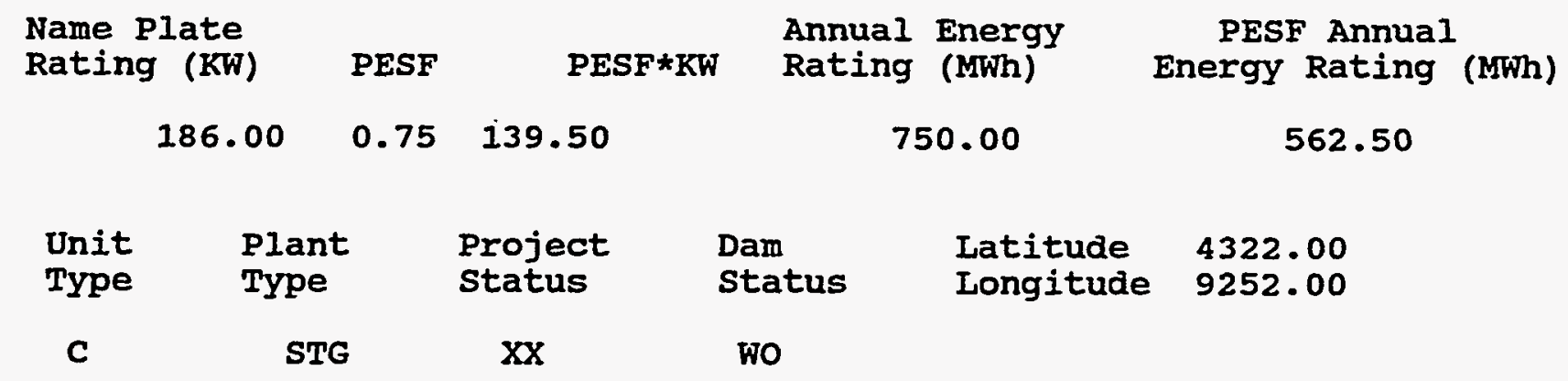

Factor

Wild/Scenic Protection Wild/Scenic Tributary or Upstream/Downstream wild/Scenic Location Cultural Value Fish Presence Value Geologic Value Historic Value other Value Recreation Value Scenic Value
Exist Prob

Factor

Exist Prob

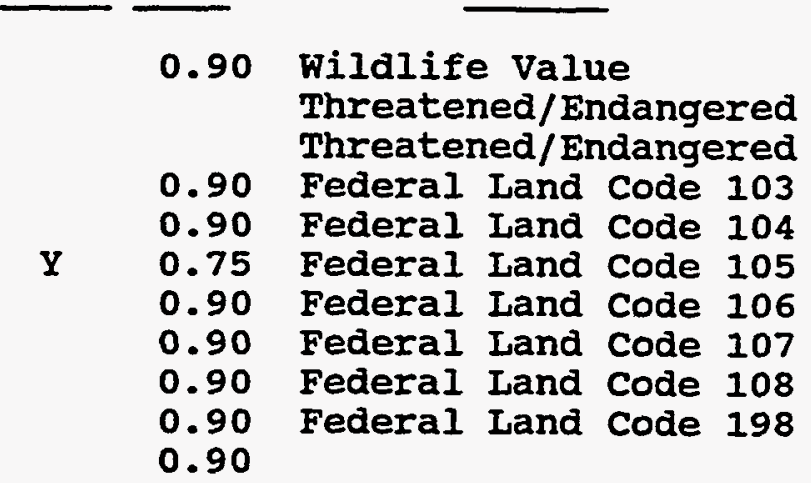

IA 
FERC

Number

Plant Name

IA101

FRASER MIILDAM

county Name

BOONE

$$
\begin{array}{cr}
\text { Class } & \text { Owner Name } \\
\text { M } & \text { TOWN OF FRASER }
\end{array}
$$

Name Plate Rating (KW)

PESF

Annual Energy PESF*KW Rating (MWh)

PESF Annual Energy Rating (MWh)

$$
760.00 \quad 0.90 \quad 684.00
$$

$\begin{array}{ll}\text { Unit } & \text { Plant } \\ \text { Type } & \text { Type }\end{array}$

$$
\text { Project }
$$
status

ROR

XX

Exist Prob

Factor

Exist Prob

Factor

Wild/Scenic Protection

wild/Scenic Tributary or Upstream/Downstream wild/Scenic Location Cultural Value Fish Presence Value Geologic Value Historic Value Other Value Recreation Value Scenic Value
Dam Latitude 4207.00

Longitude 9358.00 state Name

IA
พO

$\begin{array}{lll}0.90 & \text { Wildlife Value } & 0.90 \\ & \text { Threatened/Endangered Fish } & 0.90 \\ 0.90 & \text { Fhreatened/Endangered Wildlife } & 0.90 \\ 0.90 & \text { Federal Land Code 103 } & 0.90 \\ 0.90 & \text { Federal Land Code 104 } & 0.90 \\ 0.90 & \text { Federal Land Code 105 } 106 & 0.90 \\ 0.90 & \text { Federal Land Code 107 } & 0.90 \\ 0.90 & \text { Federal Land Code 108 } & 0.90 \\ 0.90 & \text { Federal Land Code 198 } & 0.90 \\ 0\end{array}$


FERC

Number

IA102
Plant Name

BOONE MILLDAM

county Name

BOONE stream

DES MOINES RIVER

River Basin

DES MOINES RIVER BASIN state

Name

IA

\section{class Owner Name \\ M CITY OF BOONE}

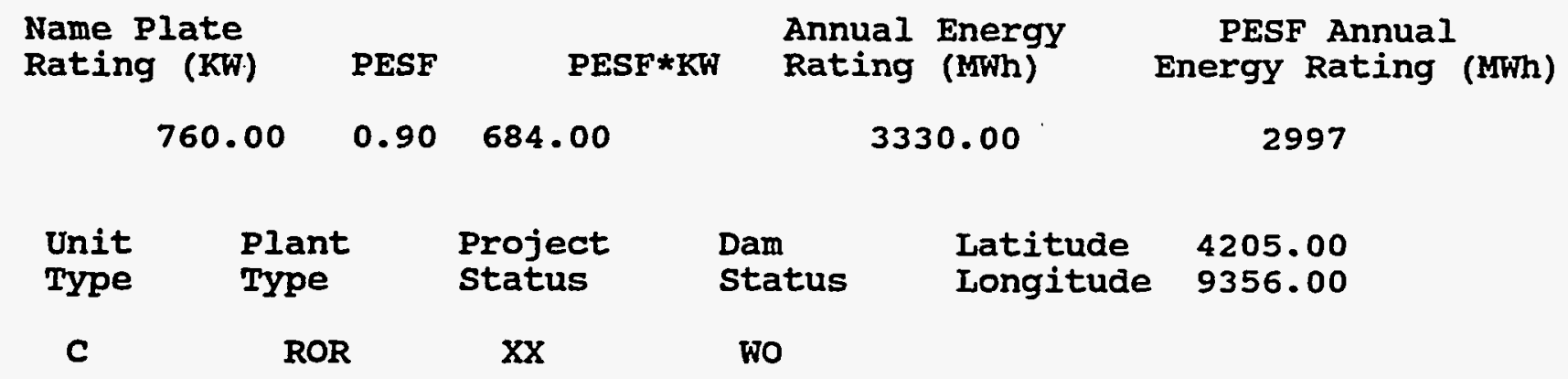

Factor

Wild/Scenic Protection Wild/Scenic Tributary or Upstream/Downstream wild/Scenic Location Cultural Value Fish Presence Value Geologic Value Historic Value other value Recreation Value Scenic Value
Exist Prob

Factor

Exist Prob

.90 Wildifife Value
Threatened/Endangered Fish Threatened/Endangered Wildilife

0.90

0.90

0.90

0.90 Federal Land Code 103

0.90 Federal Land Code 104

0.90 Federal Land Code 105

0.90 Federal Land Code 106

0.90 Federal Land Code 107

0.90 Federal Land Code 108

0.90 Federal Land Code 198

0.90
0.90

0.90

0.90

0.90

0.90

0.90

0.90 
FERC

Number

IA103
Plant Name

SCOTT STREET DAM

County Name

POIK
Stream

DES MOINES RIVER

River Basin

DES MOINES IRIVER BASIN
State

Name

IA

$$
\begin{array}{cc}
\text { Class } & \text { Owner Name } \\
\text { M } & \text { CITY OF DES MOINES }
\end{array}
$$

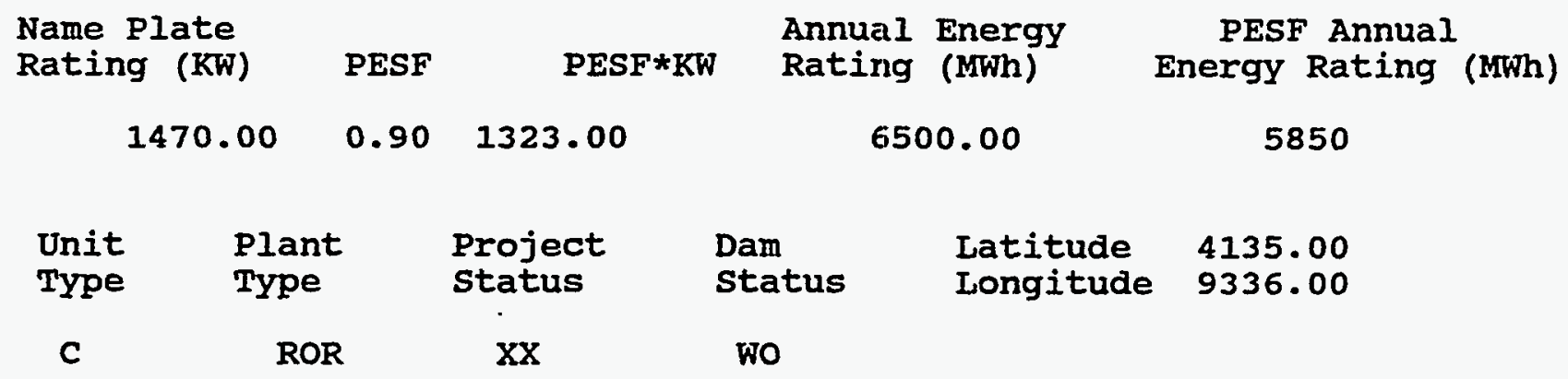

Factor

Wild/Scenic Protection

wild/Scenic Tributary or Upstream/Downstream wild/Scenic Location Cultural value

Fish Presence Value Geologic Value Historic Value Other Value Recreation value Scenic Value
Exist Prob

Factor

Exist Prob

0.90 Wildlife Value

Threatened/Endangered Fish Threatened/Endangered Wildlife

0.90

0.90

0.90

0.90 Federal Land Code 103

0.90 Federal Land Code 104

0.90

0.90

0.90 Federal Land Code 105

0.90 Federal Land Code 106

0.90

0.90 Federal Land Code 107

0.90

0.90

0.90

Federal Land Code 108

0.90

0.90 Federal Land Code 198

0.90 
FERC

Number

IA104
Plant Name

FERTILE MILLDAM

county Name

WORTH

\section{Stream}

WINNEBAGO RIVER

River Basin

MINOR RIVER BASIN state

Name

IA

$\begin{array}{cc}\text { Class } & \text { Owner Name } \\ \text { M } & \text { WORTH COUNTY CONSERVATION BOARD }\end{array}$

$\begin{array}{rrrcr}\begin{array}{l}\text { Name Plate } \\ \text { Rating (KW) }\end{array} & \text { PESF } & \text { PESF*KW } & \begin{array}{l}\text { Annual Energy } \\ \text { Rating }\end{array} & \begin{array}{c}\text { PESF Annual } \\ \text { (MWh) }\end{array} \\ 130.00 & 0.90 & 117.00 & 570.00 & 513\end{array}$

\begin{tabular}{|c|c|c|c|c|c|}
\hline $\begin{array}{l}\text { Unit } \\
\text { Type }\end{array}$ & $\begin{array}{l}\text { Plant } \\
\text { Type }\end{array}$ & $\begin{array}{l}\text { Project } \\
\text { status }\end{array}$ & $\begin{array}{l}\text { Dam } \\
\text { status }\end{array}$ & $\begin{array}{l}\text { Latitude } \\
\text { Longitude }\end{array}$ & $\begin{array}{l}4316.00 \\
9325.00\end{array}$ \\
\hline
\end{tabular}

C

ROR

$\mathrm{XX}$

Wo

Factor

Exist Prob

0.90

Wildlife Value Threatened/Endangered Fish Threatened/Endangered Wildlife

0.90 Federal Land Code 103

0.90 Federal Land Code 104

0.90 Federal Land Code 105

0.90 Federal Land Code 106

0.90 Federal Land Code 107

0.90 Federal Land Code 108

0.90 Federal Land Code 198
Exist Prob

$\begin{array}{ll} & \\ & \\ \text { Fish } & 0.90 \\ \text { Wildlife } & 0.90 \\ & 0.90 \\ & 0.90 \\ & 0.90 \\ & 0.90 \\ & 0.90 \\ & 0.90 \\ & 0.90 \\ & 0.90\end{array}$

0.90

0.90

0.90

Scenic Value 
DATE: $11 / 24 / 95$

FERC

Number

Plant Name

IA105 FORT ATKINSON

County Name

WINNESHIEK

class

Ormer Name

I WEIST FEED MILI COMPANY

PAGE NO: 60

$\begin{array}{lc}\text { Stream } & \begin{array}{c}\text { State } \\ \text { Name }\end{array} \\ \text { TURKEY RIVEIR } & \text { IA } \\ \text { River Basin } & \\ \text { MINOR RIVER BASIN } & \end{array}$

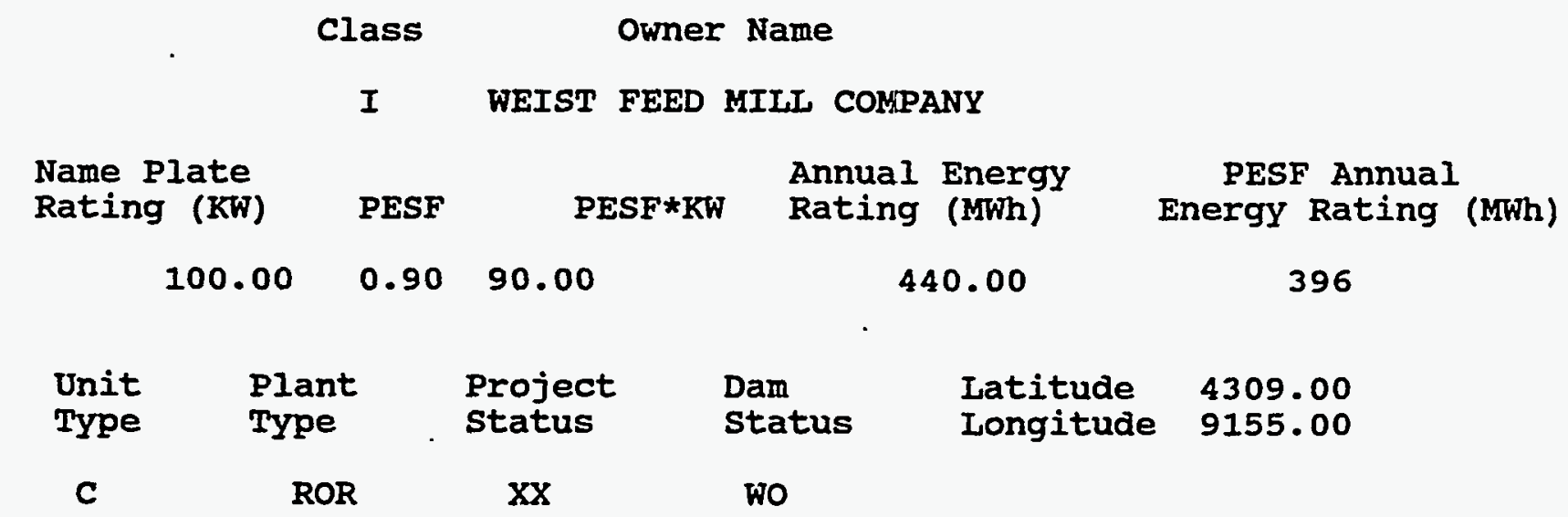

Factor

Exist Prob

Factor

Exist Prob

Wild/Scenic Protection

wild/Scenic Tributary or Upstream/Downstream Wild/Scenic Location Cultural Value Fish Presence Value Geologic Value Historic Value Other Value Recreation Value Scenic Value

0.90

0.90

0.90

0.90

0.90

0.90

0.90

0.90

0.90

0.90 
DATE: $11 / 24 / 95$

FERC

Number

IA106

LEHIGH
Plant Name

County Name

WEBSTER
PAGE NO: 61

State . Name

IA

DES MOINES RIVER

River Basin

DES MOINES RIVER BASIN

Name Plate

Rating (KW)

810.00 class

M

CITY OF LEHIGH
Annual Energy PESF*KW Rating (MWh)
PESF Annual Energy Rating (MWh)

3240

\begin{tabular}{|c|c|c|c|c|c|}
\hline $\begin{array}{l}\text { Unit } \\
\text { Type }\end{array}$ & $\begin{array}{l}\text { Plant } \\
\text { Type }\end{array}$ & $\begin{array}{l}\text { Project } \\
\text { Status }\end{array}$ & $\begin{array}{l}\text { Dam } \\
\text { status }\end{array}$ & $\begin{array}{l}\text { Latitude } \\
\text { Longitude }\end{array}$ & $\begin{array}{l}4221.00 \\
9402.00\end{array}$ \\
\hline C & ROR & $x x$ & พо & & \\
\hline
\end{tabular}

Factor

Wild/Scenic Protection Wild/Scenic Tributary or Upstream/Downstream wild/Scenic Location Cultural Value Fish Presence Value Geologic Value Historic Value other Value Recreation Value Scenic Value
Exist Prob

Factor

\subsection{0 \\ 0.90 \\ 0.90 \\ 0.90 \\ 0.90 \\ 0.90 \\ 0.90 \\ 0.90}

Wildlife Value Threatened/Endangered Fish Threatened/Endangered Wildlife

Federal Land Code 103

Federal Land Code 104

Federal Land Code 105

Federal Land Code 106

Federal Land Code 107

Federal Land Code 108

0.90
Exist Prob

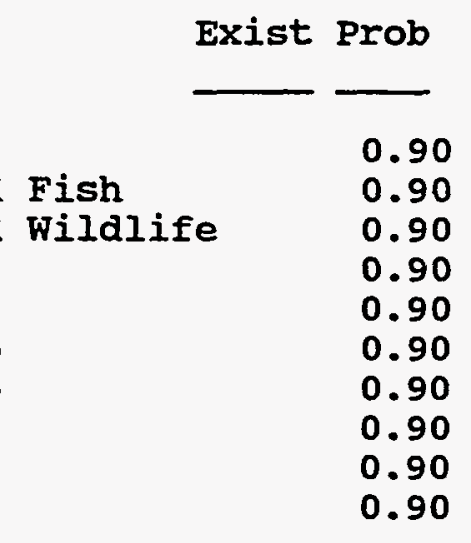

0.90

0.90

0.90

0.90

0.90

0.90

0.90
Federal Land Code 198 
DATE: $11 / 24 / 95$

PAGE NO: 62

FERC

Number

Plant Name

IA107 ROCKFORD

County Name

FLOYD

\section{Class}

M FLOYD COUNTY CONSERVATION BOARD

Name Plate

Rating ( $\mathrm{KW}$ )

150.00

PESF

PESF*KW

Annual Energy

Rating (MWh)

660.00

$0.90 \quad 135.00$

\begin{abstract}
Plant
Type
\end{abstract}

Project

status

$\mathrm{XX}$

Status

Latitude

Longitude

4303.00

พo
594
State

Name

IA
PESF Annual

Energy Rating (MWh)
ROR

c

Factor

Wild/Scenic Protection

Wild/Scenic Tributary or Upstream/Downstream wild/Scenic Location Cultural Value Fish Presence Value Geologic Value Historic Value other Value Recreation Value Scenic Value
Exist Prob

0.90

wildlife Value Threatened/Endangered Fish Threatened/Endangered wildlife

0.90 Federal Land Code 103

0.90 Federal Land Code 104

0.90 Federal Land Code 105

0.90 Federal Land Code 106

0.90 Federal Land Code 107

0.90 Federal Land Code 108

0.90 Federal Land Code 198

0.90

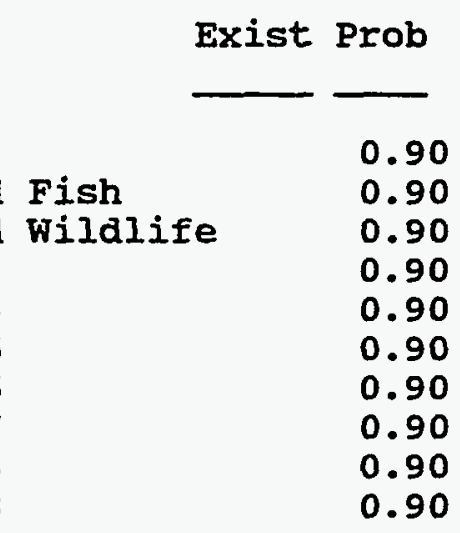


DATE: $11 / 24 / 95$

FERC

Number

Plant Name

IA108 FORT DODGE MIILDAM

county Name

WEBSTER
PAGE NO: 63

State

Name

DES MOINES RIVER

IA

River Basin

DES MOINES RIVER BASIN

$\begin{array}{cc}\text { Class } & \text { Owner Name } \\ \text { M } & \text { CITY OF FORT DODGE }\end{array}$

Name Plate

Rating ( $\mathrm{KW}$ )
Annual Energy Rating (MWh)

7700.00
PESF Annual Energy Rating (MWh)
1760.00

PESF PESF*KW

$0.90 \quad 1584.00$

Unit
Type

Plant

Type

c

STG
Project status

$\mathrm{XX}$ $\begin{array}{ll}\text { Dam } & \text { Latitude } \\ \text { Status } & \text { Longitude }\end{array}$

พอ
Factor

Wild/Scenic Protection

wild/Scenic Tributary or Upstream/Downstream wild/Scenic Location

Cultural Value

Fish Presence Value

Geologic Value

Historic Value

Other Value

Recreation value

Scenic Value
Exist Prob

Factor

Exist Prob

0.90

Wildlife Value

Threatened/Endangered Fish

Threatened/Endangered Wildlife

0.90 Federal Land Code 103

0.90 Federal Land Code 104

0.90 Federal Land Code 105

0.90 Federal Land Code 106

0.90 Federal Land Code 107

0.90 Federal Land Code 108

0.90 Federal. Land Code 198

0.90
6930

4029.00

9411.00 
DATE: $11 / 24 / 95$

FERC

Number

IA109

BONAPARTE

County Name

VAN BUREN

Class

Owner Name

M CITY OF BONAPARTE

Name Plate

Rating (KW)

PESF PESF*KW

Annual Energy

Rating (MWh)

2990.00

$0.90 \quad 2691.00$

13100.00

PESF Annual

Energy Rating (MWh)

11790

$\begin{array}{cccccc}\text { Unit } & \text { Plant } & \text { Project } & \text { Dam } & \text { Latitude } & 4042.00 \\ \text { Type } & \text { Type } & \text { Status } & \text { Status } & \text { Longitude } 9148.00 \\ \text { C } & \text { STG } & \mathrm{XX} & \text { wo } & & \end{array}$

Factor

Exist Prob

wild/Scenic Protection

wild/Scenic Tributary or

Upstream/Downstream

wild/Scenic Location

Cultural value

Fish Presence Value

Geologic Value

Historic Value

other Value

Recreation value

Scenic Value
Factor

Exist Prob
0.90 Wildlife Value Threatened/Endangered Fish Threatened/Endangered Wildlife
0.90 Federal Land Code 103
0.90 Federal Land Code 104
0.90 Federal Land Code 105
0.90 Federal Land Code 106
0.90 Federal Land Code 107
0.90 Federal Land Code 108
0.90 Federal Land Code 198

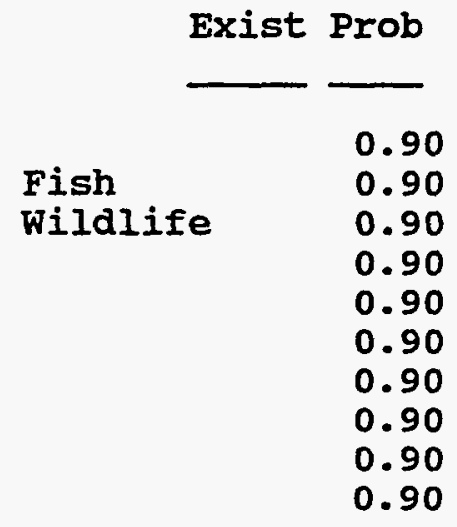

Name

IA 
DATE: $11 / 24 / 95$

PAGE NO: 65

FERC

Number

Plant Name

stream

State

IA110 LITTLE SIOUX DAM

LITTLE SIOUX RIVER

Name

county Name

River Basin

HARRISON

MINOR RIVER BASIN

Class Owner Name

M IITTLE SIOUX INTER COUNTY DRAINAGE

Name Plate

Rating (KW)

PESF

Annual Energy

PESF * KW

Rating (MWh)

PESF Annual

690.00

$0.90 \quad 621.00$

3020.00

$\begin{array}{ll}\text { Unit } & \text { Plant } \\ \text { Type } & \text { Type }\end{array}$

C

ROR

IA

2718

\section{Energy Rating (WWh)}

Factor

Wild/Scenic Protection

Wild/Scenic Tributary or Upstream/Downstream wild/Scenic Location Cultural Value

Fish Presence Value

Geologic Value

Historic Value

other Value

Recreation Value

Scenic Value
Project

status

$\mathrm{XX}$
Dam

status

พั
Exist Prob

Factor

Latitude

Longitude

4151.00

9559.00 
R E S O U R C E D A T A B A S E I I S T I N G

DATE: $11 / 24 / 95$

PAGE NO: 66

FERC

Number

Plant Name

Stream

State

Name

IA111 AMANA DAM

IOWA RIVER

IA

County Name

River Basin

IOWA

IOWA RIVER BASIN

class Owner Name

R AMANA SOCIETY SERVICE COMPANY

Name Plate

Rating (KW)

PESF

Annual Energy

PESF $* K W$ Ratirig (MWh)

PESF Annual

280.00

$0.90 \quad 252.00$

1230.00

Energy Rating (MWh)

1107

\begin{tabular}{|c|c|c|c|c|c|}
\hline $\begin{array}{l}\text { Unit } \\
\text { Type }\end{array}$ & $\begin{array}{l}\text { Plant } \\
\text { Type }\end{array}$ & $\begin{array}{l}\text { Project } \\
\text { status }\end{array}$ & $\begin{array}{l}\text { Dam } \\
\text { status }\end{array}$ & $\begin{array}{l}\text { Latitude } \\
\text { Longitude }\end{array}$ & $\begin{array}{l}4147.00 \\
9158.00\end{array}$ \\
\hline C & ROR & & $\mathbf{W}$ & & \\
\hline
\end{tabular}

Factor

Exist Prob

Factor

Exist Prob

Wild/Scenic Protection

wild/Scenic Tributary or Upstream/Downstream wild/Scenic Location Cultural Value

Fish Presence Value Geologic Value

Historic Value

other Value

Recreation Value

Scenic Value

0.90 Wildiife Value

Threatened/Endangered Fish

Threatened/Endangered Wildlife

0.90 Federal Land Code 103

0.90 Federal Land Code 104

0.90 Federal Land Code 105

0.90 Federal Land Code 106

0.90 Federal Land Code 107

0.90 Federal Land Code 108

0.90 Federal Land Code 198

0.90
0.90

0.90

0.90

0.90

0.90

0.90

0.90

0.90

0.90

0.90 
FERC

Number

IA112 IYNNVIILE DAM

county Name

JASPER

\section{Class}

M JASPER COUNTY CONSERVATION BOARD

Name Plate Rating (KW)

PESF

150.00

$0.90 \quad 135.00$

PESF*KW

Annual Energy

Rating (MWh)

660.00

Unit

Type

Plant

Project status

C

ROR

$\dot{x} \mathbf{x}$

Exist Prob

Factor

Wild/Scenic Protection

Wild/Scenic Tributary or Upstream/Downstream Wild/Scenic Location Cultural value

Fish Presence Value

Geologic Value

Historic Value

Other Value

Recreation Value

Scenic Value

$\begin{array}{lll}\text { Dam } & \text { Latitude } & 4134.00 \\ \text { Status } & \text { Longitude } & 9246.00\end{array}$

พั
State

Name

IA
594

PESF Annual
Energy Rating (MWh) 
DATE: $11 / 24 / 95$

FERC

Number

$\operatorname{IAI13}$

ANAMOSA MIILLDAM

County Name

JONES
PAGE NO: 68

state

Name

WAPSIPINICON RIVER

IA
River Basin

MINOR RIVER BASIN

\section{class Owner Name}

$p$ IES UTITIES

Name Plate

Rating (KW)

PESF

Annual Energy

PESF Annual

700.00

0.90

630.00

3070.00

2763

$\begin{array}{lll}\text { Unit } & \text { Plant } & \text { Project } \\ \text { Type } & \text { Type } & \text { Status }\end{array}$

C

ROR

$\begin{array}{lll}\text { Dam } & \text { Latitude } & 4206.00 \\ \text { Status } & \text { Longitude } & 9117.00\end{array}$

W

Factor

Wild/Scenic Protection

Wild/Scenic Tributary or Upstream/ Downstream wild/Scenic Location Cultural Value Fish Presence Value Geologic Value Historic Value other Value Recreation Value Scenic Value
Exist Prob

Factor

Exist Prob

\subsection{0}

Wildlife Value

Threatened/Endangered Fish Threatened/Endangered wildlife

0.90 Federal Land Code 103

0.90 Federal Land Code 104

0.90 Federal Land Code 105

0.90 Federal Land Code 106

0.90

0.90

Federal Land Code 107

Federal Land Code 108

0.90

Federal Land Code 198
0.90

0.90

0.90

0.90

0.90

0.90

0.90

0.90

0.90

0.90 
DATE: $11 / 24 / 95$

FERC

Number

Plant Name

IA114 OXFORD MILLS

County Name

JONES
PAGE NO: 69

State

Name

WAPSIPINICON RIVER

IA
River Basin

MINOR RIVER BASIN

\section{Class Owner Name \\ $P$ IES UTILITIES}

Name Plate

Rating (KW)

PESF

PESF*KW

Annual Energy

Rating ( $\mathrm{MWh}$ )

PESF Annual

Energy Rating (MWh)

$$
570.00 \quad 0.90 \quad 513.00
$$

2500.00

2250

Unit
Type

Plant

Project

status

c

ROR

$\mathrm{XX}$

Exist Prob

Factor

Wila/Scenic Protection

Wild/Scenic Tributary or Upstream/Downstream wila/Scenic Location Cultural Value

Fish Presence Value

Geologic Value

Historic Value

other Value

Recreation Value

Scenic Value

$\begin{array}{lll}\text { Dam } & \text { Latitude } & 4158.00 \\ \text { Status } & \text { Longitude } & 9057.00\end{array}$

พอ 
FERC

Number

Plant Name

IAI15

PALISADES-KEPLER

County Name

IINN

class

Owner Name

M IOWA CONSERVATION COMMISSION

Name Plate

Rating (KW)

PESF

Annual Energy

PESF*KW

Rating (MWh)

PESF Annual

2010.00

$0.90^{\circ} 1809.00$

8530.00

677

$\begin{array}{lll}\text { Unit } & \text { Plant } & \text { Project } \\ \text { Type } & \text { Type } & \text { Status }\end{array}$

C

Factor

wila/Scenic Protection

wila/Scenic Tributary or

Upstream/Downstream

Wild/scenic Location

Cultural Value

Fish Presence Value

Geologic Value

Historic Value

other Value

Recreation Value

Scenic Value
Exist Prob

0.90

Wildlife Value Threatened/Endangered Fish Threatened/Endangered wildlife

0.90 Federal Land Code 103

0.90 Federal Land Code 104

0.90 Federal Land Code 105

0.90 Federal Land Code 106

0.90 Federal Land Code 107

0.90 Federal Land Code 108

0.90 Federal Land Code 198
State

Name

IA Energy Rating (MWh)

0.90

Exist Prob

0.90

0.90

0.90

0.90

0.90 
FERC

Number

Plant Name

IA116 TROY MILLS

County Name

LINN

\section{Stream}

WAPSIPINICON RIVER

River Basin

MINOR RIVER BASIN
State

Name

IA

\section{Class Owner Name}

$P$ IES UTIIITIES

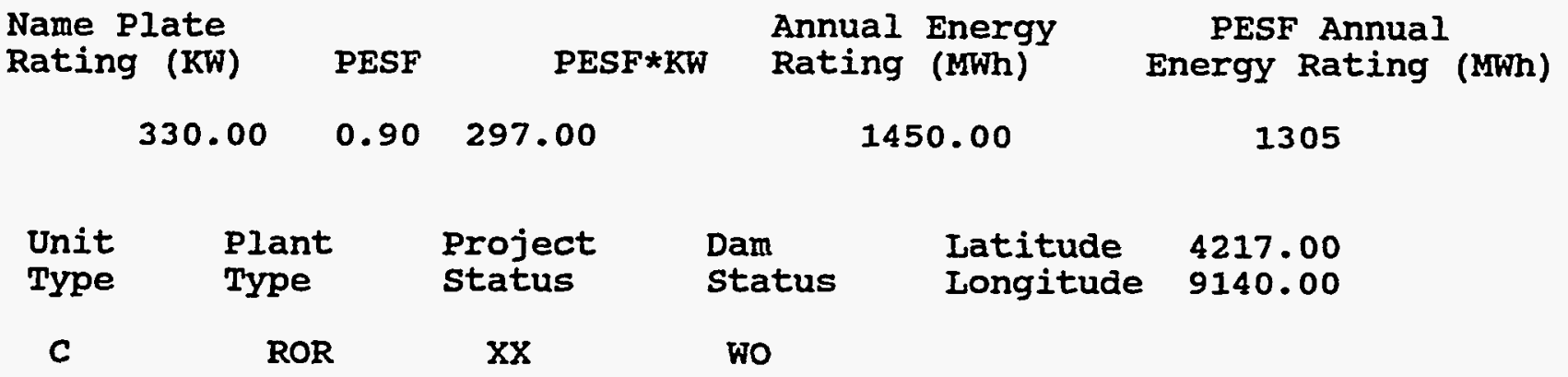

Factor

Exist Prob

Factor

Exist Prob

Wild/scenic Protection

Wild/Scenic Tributary or Upstream/Downstream wild/Scenic Iocation 0.90 Wildlife Value
Threatened/Endangered Fish

0.90

0.90 Threatened/Endangered Wildlife

0.90

0.90 Federal Land Code 103

0.90

0.90 Federal Land Code 104

0.90

0.90 Federal Land Code 105

0.90

0.90 Federal Land Code 106

0.90

0.90 Federal Land Code 107

0.90

0.90 Federal Land Code 108

0.90

0.90

Federal Land Code 198

0.90 
R E S O U R C E

DATE: $11 / 24 / 95$
D A T A B A $\mathrm{E}$

I I S T I N G
FERC

Number

IA 117
Plant Name

WAVERLY MILLDAM

County Name

BREMER
PAGE NO: 72
State

Name

CEDAR RIVER

IA
River Basin

MINOR RIVER BASIN

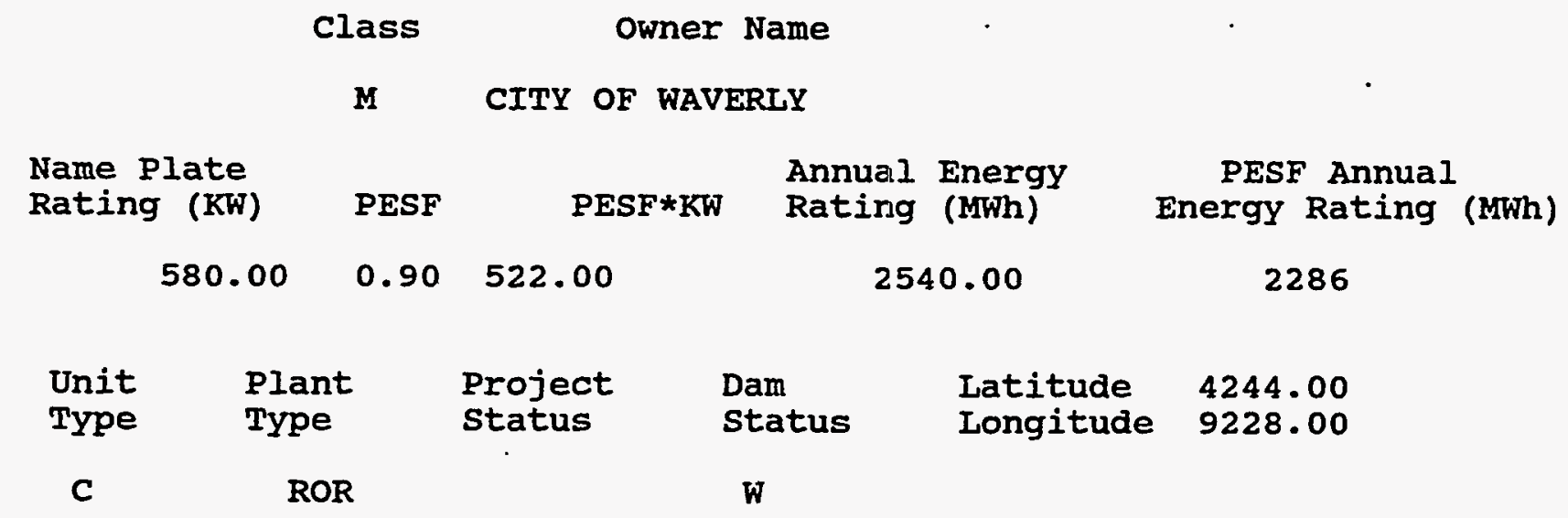

Factor

Wild/Scenic Protection

wild/Scenic Tributary or Upstream/Downstream wild/Scenic Location Cultural value Fish Presence Value Geologic. Value Historic Value Other Value Recreation value Scenic Value
Exist Prob

Factor

Exist Prob

\subsection{Wildlife value}

Threatened/Endangered Fish

Threatened/Endangered Wildlife

0.90 Federal Land Code 103

0.90 Federal Land Code 104

0.90 Federal Land code 105

0.90 Federal Irand code 106

0.90 Federal Land code 107

0.90 Federal Land Code 108

0.90

0.90
Exist Prob

0.90

0.90

0.90

0.90

0.90

0.90

0.90

0.90

0.90

0.90 
DATE: $11 / 24 / 95$

PAGE NO: 73

FERC

Number

IA118 QUASQUETON DAM

county Name
BUCHANAN

Plant Name

\author{
Name
}

$$
\begin{array}{cc}
\text { Class } & \text { Owner Name } \\
\text { M } & \text { CITY OF QUASQUETON }
\end{array}
$$

state Name

IA

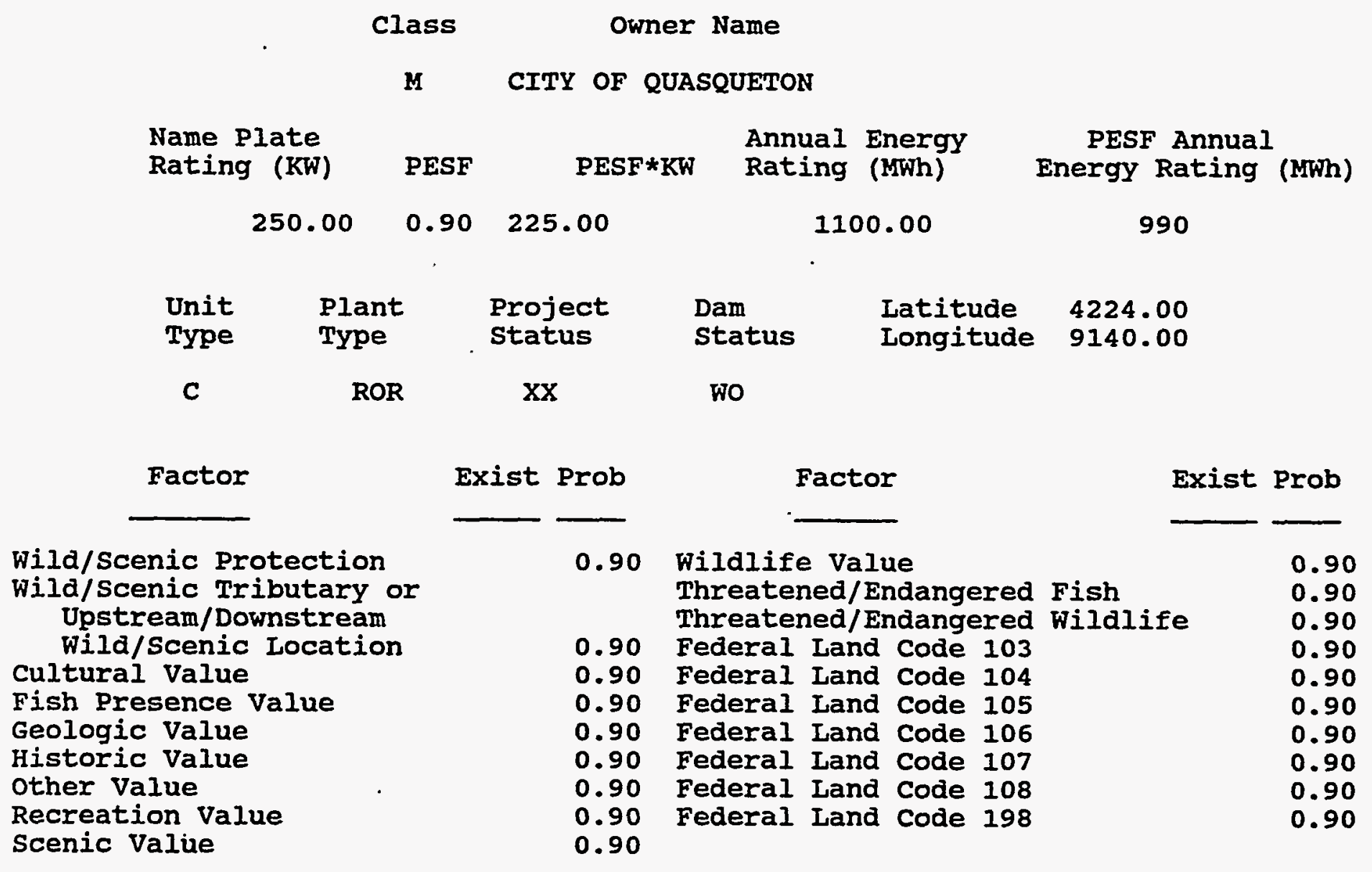

Wild/Scenic Protection

wild/Scenic Tributary or Upstream/Downstream wild/Scenic Location Cultural value Fish Presence Value Geologic Value Historic Value other Value Recreation value Scenic Value
WAPSIPINICON RIVER

$$
\text { River Basin }
$$

MINOR RIVER BASIN 
FERC Number

IA119
Plant Name MASON CITY MIILDAM

County Name

CERRO GORDO
Stream

WINNEBAGO RIVER

River Basin

MINOR RIVER BASIN
State

Name

IA

\section{Class Owner Name \\ I AMERICAN CRYSTAL SUIGAR}

Name Plate

Rating (KW)

PESF

Annual Energy

PESF*KW

Rating (MWh)

PESF Annual

100.00

0.90

90.00

440.00

Energy Rating (MWh)

$\begin{array}{cccccc}\text { Unit } & \text { Plant } & \text { Project } & \text { Dam } & \text { Latitude } & 4311.00 \\ \text { Type } & \text { Type } & \text { Status } & \text { Status } & \text { Longitude } 9312.00 \\ \text { C } & \text { ROR } & \text { XX } & \text { WO } & \end{array}$

Factor

Wild/Scenic Protection

Wild/Scenic Tributary or Upstream/Downstream wild/Scenic Location Cultural value Fish Presence Value Geologic Value Historic Value other Value Recreation Value Scenic Value
Exist Prob

Factor

Exist Prob

\subsection{0}

Wildlife Value

Threatened/Endangered Fish

Threatened/Endangered Wildlife

0.90 Federal Land Code 103

0.90 Federal Land Code 104

0.90 Federal Land Code 105

0.90 Federal Land Code 106

0.90 Federal Land Code 107

0.90 Federal Land Code 108

0.90 Federal Land Code 198

0.90
0.90

0.90

0.90

0.90

0.90

0.90

0.90

0.90

0.90

0.90 
DATE: $11 / 24 / 95$

PAGE NO: 75

FERC

Number

Plant Name

Stream

state

IA121 KLONDIKE MIILDAM

BIG SIOUX RIVER

Name

county Name

River Basin

IYON

MINOR RIVER BASIN

\begin{tabular}{cc} 
class & \multicolumn{2}{c}{ Owner Name } \\
M & STATES OF IOWA \& SOUTH DAKOTA
\end{tabular}

Name Plate

Rating (KW)

PESF

Annual Energy

PESF*KW Rating (MWh)

PESF Annual

$440.00 \quad 0.90 \quad 396.00$

1930.00

$\begin{array}{cccccc}\text { Unit } & \text { Plant } & \text { project } & \text { Dam } & \text { Latitude } & 4323.00 \\ \text { TYpe } & \text { Type } & \text { Status } & \text { Status } & \text { Longitude } & 9631.00 \\ \text { C } & \text { ROR } & \text { XX } & \text { WO } & \end{array}$

Factor

Exist Prob

Factor

Exist Prob

Wild/Scenic Protection

Wild/Scenic Tributary or Upstream/Downstream wild/Scenic Location Cultural value

Fish Presence Value Geologic Value

Historic Value Other Value

Recreation Value

Scenic Value
0.90 Wildlife Value Threatened/Endangered Fish Threatened/Endangered wildlife

0.90 Federal Land Code 103

0.90 Federal Land Code 104

0.90 Federal Land Code 105

0.90 Federal Land Code 106

0.90 Federal Land Code 107

0.90 Federal Land Code 108

0.90 Federal Land Code 198
IA

\section{7}

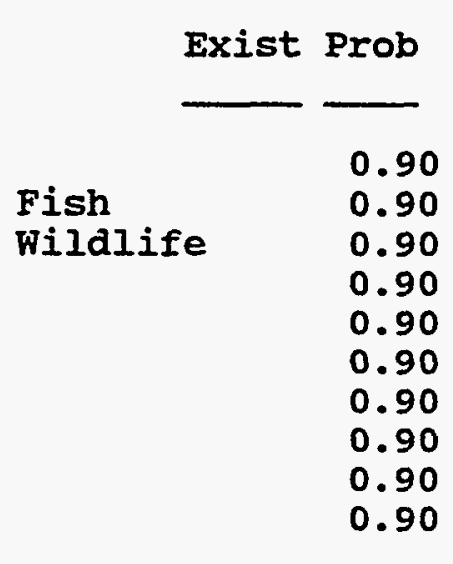


FERC Number

IA122
Plant Name

CLERMONT MIILDAM

County Name

FAYETTE
Stream

TURKEY RIVER

River Basin

MINOR RIVER BASIN
State

Name

IA

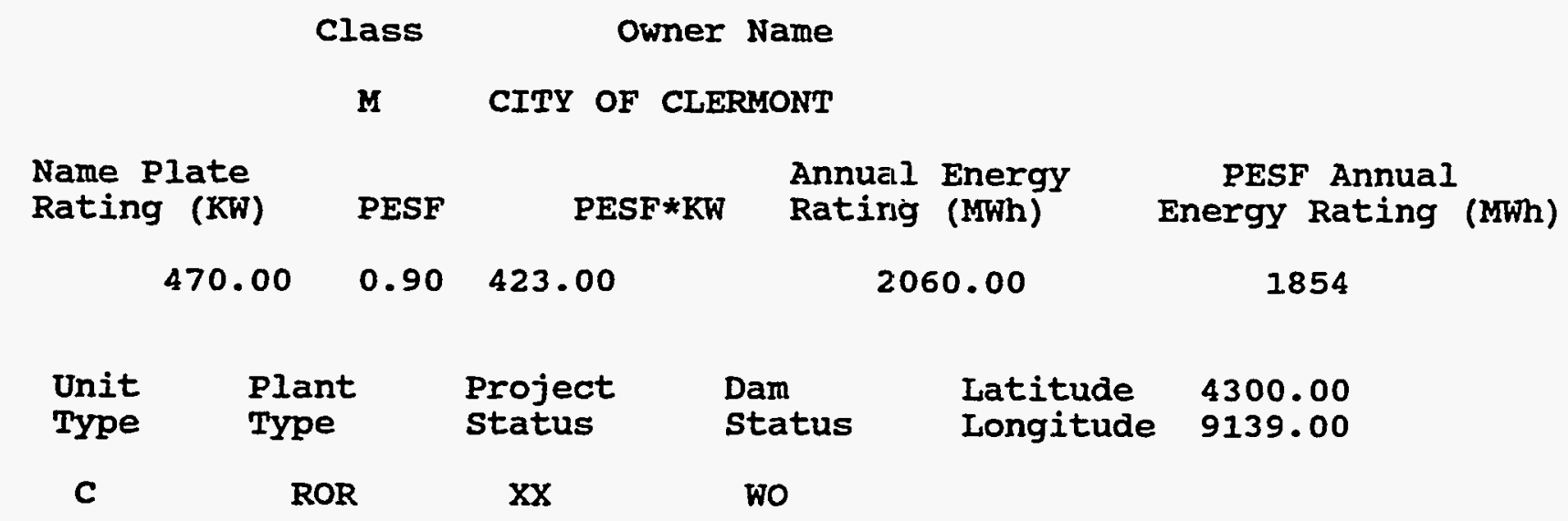

Factor

Exist Prob

Factor

Exist Prob

Wild/Scenic Protection

Wild/Scenic Tributary or

0.90 Wildlife Value

Threatened/Endangered Fish

Upstream/Downstream

Threatened/Endangered $\cdot$ Wildlife

0.90

wild/Scenic Location

Cultural Value

$\begin{array}{ll}0.90 & \text { Federal Land Code } 103 \\ 0.90 & \text { Federal Land Code } 104\end{array}$

0.90

0.90 Federal Land Code 104

0.90

0.90 Federal Land Code 106

0.90

Geologic Value

0.90 Federal Land Code 107

0.90

Historic Value

other Value

Recreation Value

0.90

0.90

0.90

Federal Land Code 108

0.90

0.90

0.90

0.90

Scenic Value

Federal Land Code 198 
DATE: $11 / 24 / 95$

FERC

Number

IA123

OTRANTO DAM

County Name

MITCHELI

Class
PAGE NO: 77

State

Name

CEDAR RIVER

IA
River Basin

MINOR RIVER BASIN

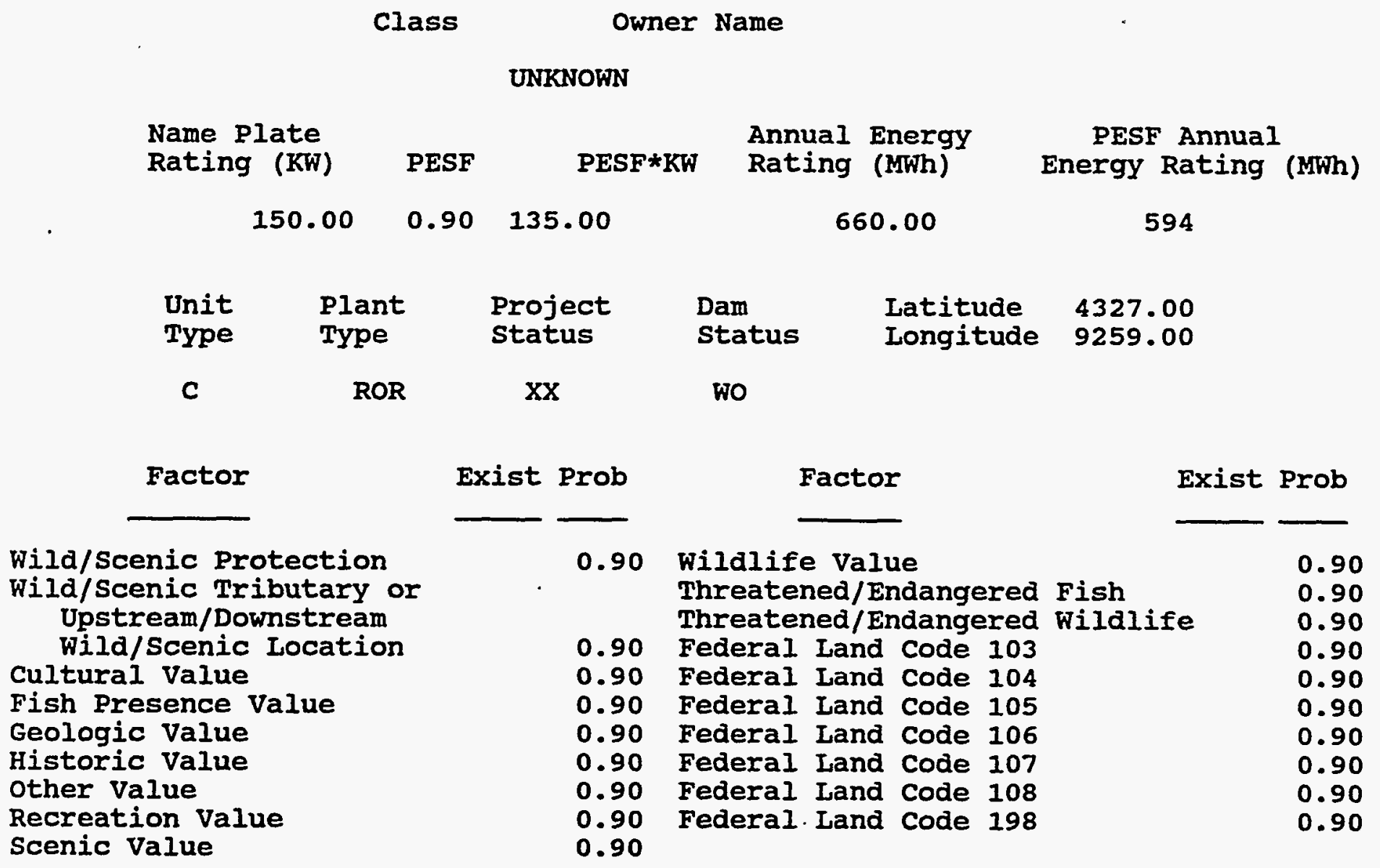


FERC

Number
Plant Name

IA124
IOWA FAILS MILIDAM

County Name

HARDIN stream

IOWA RIVER

River Basin

IOWA RIVER BASIN state

Name

IA

\section{Class Owner Name \\ $P$ IES UTILITIES}

Name Plate

Rating (KW)

PESF

Annual Energy

PESF $\star K W$

Rating (MWh)

PESF Annual

$$
540.00 \quad 0.90 \quad 486.00
$$

$$
2370.00
$$

\begin{tabular}{|c|c|c|c|c|c|}
\hline $\begin{array}{l}\text { Unit } \\
\text { Type }\end{array}$ & $\begin{array}{l}\text { Plant } \\
\text { Type }\end{array}$ & $\begin{array}{l}\text { Project } \\
\text { Status }\end{array}$ & $\begin{array}{l}\text { Dam } \\
\text { Status }\end{array}$ & $\begin{array}{l}\text { Latitude } \\
\text { Longitude }\end{array}$ & $\begin{array}{l}4231.00 \\
9315.00\end{array}$ \\
\hline C & ROR & zz & พ & & \\
\hline
\end{tabular}

2133

Factor

Exist Prob

\subsection{0}

Wild/Scenic Protection

Wild/Scenic Tributary or Upstream/Downstream wild/Scenic Location Cultural Value

Fish Presence Value

Geologic Value

Historic Value

other Value

Recreation Value

Scenic Value

0.90
Factor

Exist Prob

\section{Wildlife Value}

Threatened/Endangered Fish

Threatened/Endangered wildlife

0.90 Federal Land Code 103

0.90 Federal Land Code 104

0.90 Federal Land Code 10.5

0.90 Federal Land Code 106

0.90 Federal Land Code 107

0.90 Federal Land Code 108

0.90 Federal Land Code 198
0.90

0.90

0.90

0.90

0.90

0.90

0.90

0.90

0.90

0.90 
DATE: $11 / 24 / 95$

FERC

Number

IA125

CLARINDA DAM

County Name

PAGE
PAGE NO: 79

State

Name

NODAWAY RIVER

IA
River Basin

MINOR RIVER BASIN

\section{Class Owner Name \\ M CITY OF CLARINDA}

Name Plate

Rating (KW)

PESF

PESF*KW

Annual Energy

Rating (MWh)

PESF Annual

140.00

$0.90 \quad 126.00$

620.00

558

Unit Plant

Type Type

C

Factor

Wild/Scenic Protection

wild/Scenic Tributary or

Upstream/Downstream

wila/Scenic Location

cultural value

Fish Presence Value

Geologic Value

Historic Value

Other Value

Recreation Value

Scenic Value

\begin{abstract}
Project Status
\end{abstract}

$\mathrm{XX}$

Exist Prob

0.90

Wildife Value Threatened/Endangered Fish Threatened/Endangered Wildlife

0.90 Federal Land Code 103

0.90 Federal Land Code 104

0.90 Federal Land Code 105

0.90 Federal Land Code 106

0.90 Federal Land Code 107

0.90 Federal Land Code 108

0.90 Federal Land Code 198
Exist Prob

0.90

0.90

0.90

0.90

0.90

0.90

0.90

0.90

0.90

0.90 


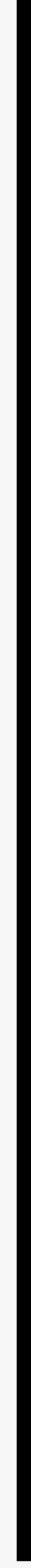

